\title{
WestVirginiaUniversity
}

THE RESEARCH REPOSITORY @ WVU

Graduate Theses, Dissertations, and Problem Reports

2020

\section{Paper-Based Point-of-Care Tools for Blood Testing}

\author{
Xuefei Gao \\ xegao@mix.wvu.edu
}

Follow this and additional works at: https://researchrepository.wvu.edu/etd

Part of the Biochemistry Commons, Nanoscience and Nanotechnology Commons, and the Semiconductor and Optical Materials Commons

\section{Recommended Citation}

Gao, Xuefei, "Paper-Based Point-of-Care Tools for Blood Testing" (2020). Graduate Theses, Dissertations, and Problem Reports. 7620.

https://researchrepository.wvu.edu/etd/7620

This Dissertation is protected by copyright and/or related rights. It has been brought to you by the The Research Repository @ WVU with permission from the rights-holder(s). You are free to use this Dissertation in any way that is permitted by the copyright and related rights legislation that applies to your use. For other uses you must obtain permission from the rights-holder(s) directly, unless additional rights are indicated by a Creative Commons license in the record and/ or on the work itself. This Dissertation has been accepted for inclusion in WVU Graduate Theses, Dissertations, and Problem Reports collection by an authorized administrator of The Research Repository @ WVU.

For more information, please contact researchrepository@mail.wvu.edu. 


\title{
Paper-Based Point-of-Care Tools for Blood Testing
}

\author{
Xuefei Gao \\ Dissertation submitted \\ to Benjamin M. Statler College of Engineering and Mineral Resources \\ at West Virginia University \\ in partial fulfillment of the requirements for the degree of \\ Doctor of Philosophy in \\ Materials Science and Engineering \\ Nianqiang $\mathrm{Wu}$, Ph.D., Chair \\ Dongling Ma, Ph.D. \\ Ever J. Barbero, Ph.D. \\ Kostas Sierros, Ph.D. \\ Terence Musho, Ph.D.
}

Department of Mechanical and Aerospace Engineering Morgantown, West Virginia

2020

Keywords: Paper-Based Devices; Biosensors; Point-of-Care; Optical Sensing; Whole Blood;

Surface-Enhanced Raman Scattering; Near-Infrared Fluorescence

Copyright 2020 Xuefei Gao 


\section{Abstract \\ Paper-Based Point-of-Care Tools for Blood Testing}

\section{Xuefei Gao}

Early detection of malignant disease is crucial for timely diagnosis and effective medical intervention, which significantly increases survival rates and reduce financial burden on patients. Biomarkers are becoming increasingly important in detection of malignant diseases, because they can be employed for indicating diseases, predicting risks and monitoring the progression of diseases. In addition, biomarkers show up at early stages of diseases in human tissues and fluids (e.g., blood, urine and saliva), which shows great promise for early disease detection. In this dissertation, paper-based lateral flow strips (PLFSs) have been developed for the detection of disease biomarkers, including protein biomarkers and microRNA (miRNA) biomarkers from clinical samples and whole blood samples. Among most of the reported biosensors, PLFSs appear to be an effective tool for providing access to point-of-care (POC) applications, due to low cost, fast response, portability and ease of use. In addition, paper is compatible with biological samples, which allows its application in analyzing various biomolecules. However, conventional PLFSs exhibit insufficient sensitivity and poor interference resistance. In particular whole blood samples, which contain numerous interference biomolecules, substantially affect detection accuracy and specificity.

In this dissertation, several strategies have been employed to solve the problems, including introducing PLFSs with ultrasensitive techniques, meanwhile modifying PLFSs with functional nanomaterials and paper accessory unit to reduce the interference biomolecules from human fluids. In summary, five chapters will be demonstrated:

(1) Surface-enhanced Raman scattering (SERS) technique modified PLFSs for protein biomarker detection in clinical blood plasma samples. In this chapter, silica coated SERS nanoparticles (NPs) have been developed for improving Raman signal and detection sensitivity, at the same time, silica coating of the SERS NPs substantially improve the stability of the NPs in complex human fluids. As a result, the developed SERS-PLFS can realize direct detection of neuron-specific enolase (NSE) from clinical blood plasma samples of traumatic brain injury (TBI) patients. The test results of the SERS-PLFSs were compatible with those from the standard enzyme-linked immunospecific assay (ELISA) method;

(2) Blood plasma separation unit (PSU) integrated PLFS for cancer protein biomarker detection from whole human blood sample. In this chapter, a paper based PSU was fabricated to efficiently retain red blood cells (RBCs) inside the unit to block their migration and meanwhile push the target protein contained plasma to the detection area of the PLFS. As a result, cancer protein biomarker-carcinoembryonic antigen (CEA) was successfully detected by the PSU-PLFS from whole blood samples;

(3) Plasmonic chip and PSU integrated PLFS for protein biomarker detection from whole blood. In order to meet the high sensitivity demand, a gold nanopyramid array functionalized chip was integrated into a PLFS for amplifying Raman signal and ultrasensitive detection of TBI protein biomarker s-100 $\beta$; while the PSU was utilized to reduce the RBCs interference from 
whole blood. As a result, compared with the result of the SERS-PLFS in Chapter 2, an improvement in LOD with two-order of magnitude was obtained;

(4) Near-infrared fluorophores (NIRFs) functionalized PLFS for miRNA detection from blood plasma. In this chapter, NIRFs encapsulated silica nanoparticles were synthesized and incorporated into a PLFS for stroke biomarker miRNA-34 detection. Compared with a single fluorescent dye, the synthesized NIRF NPs encapsulated numerous fluorophores into one single silica nanoparticle, exhibiting amplified luminescent intensity. Moreover, the NIRF nanoparticles minimized the fluorescent background from biological matrices and test strip materials, which elevates signal-to-noise ratio and anti-interference capacity;

(5) Duplex specific nuclease (DSN) based signal amplification strategy modified PLFS for ultrasensitive miRNA detection in blood plasma. In order to meet the high sensitivity demand of miRNA-34 measurement from the clinical blood plasma of stroke patient, the developed NIRFsPLFS in Part 4 was further elevated with DSN modification for amplifying fluorescent signal. As a result, the DSN-PLFS exhibited an improvement in detection sensitivity with two-orders of magnitude in blood plasma. 


\section{ACKNOWLEDGEMENT}

Foremost, I would like to express my sincere gratitude to my supervisor Prof. Nianqiang $\mathrm{Wu}$ for his encouragement, continuous support and guidance for my studies. My sincere thanks also go to my PhD dissertation committee: Prof. Dongling Ma, Prof. Ever J. Barbero, Prof. Kostas Sierros and Prof. Terence Musho for their valuable time, encouragement and insightful advice. I would also like to express my sincere thanks to Prof. Yon Rojanasakul, Prof. James Simpkins and Prof. Yuxin Liu for their support in my research.

My sincere thanks also go to all my labmates I have worked with at West Virginia University: Dr. Hui Yang, Dr. Peng Zheng, Dr. Sujan Kasani, Joseph Bright, Botong Liu, Prof. Haibin Tang, Prof. Zhulin Huang, Prof. Jianhui Yang, Jennifer Boryczka, Kathrine Curtin, Nicholas Winch, Dr. Jiangtian Li, Prof. Scott Cushing, Yang He and Shimeng Hao.

My deepest gratitude and thanks goes to my husband Wei $\mathrm{Li}$, to his highest regards, understanding and the strongest support. Special thanks to my parents for their support and encouragement during the whole period of my studies.

Xuefei Gao

West Virginia University

March 2020 


\section{Table of Contents}

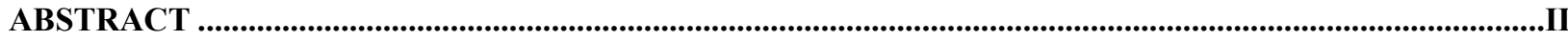

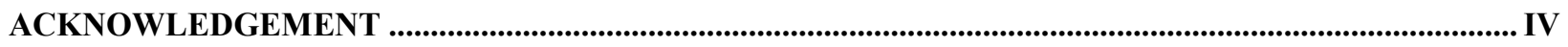

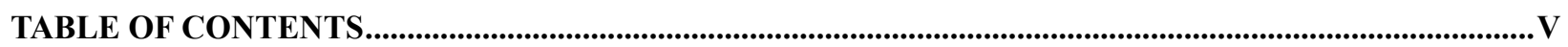

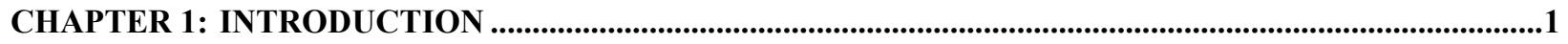

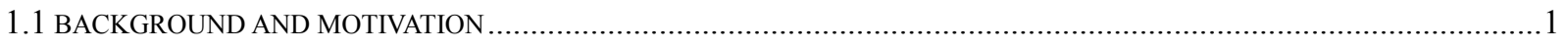

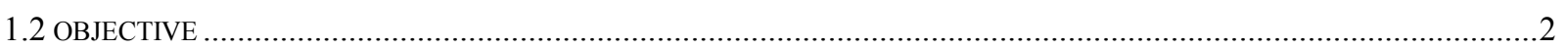

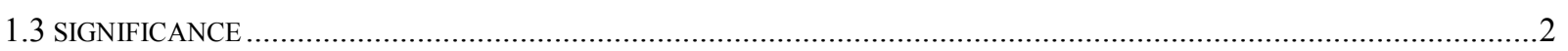

1.4 INNOVATION

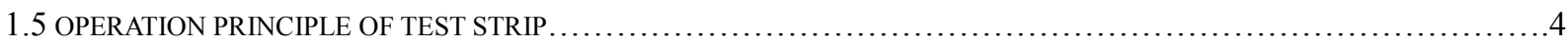

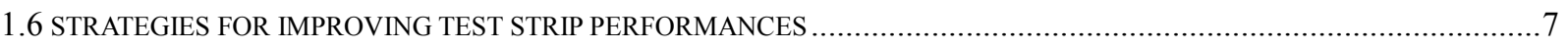

1.7 TECHNIQUE OF SURFACE-ENHANCED RAMAN SCATTERING ...............................................................................

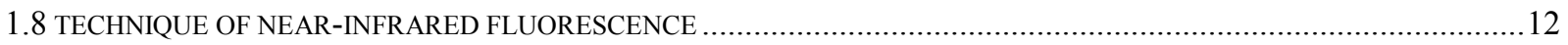

CHAPTER 2: SURFACE ENHANCED RAMAN SACTTERING TEST STRIP FOR PLASMA TESTING .14

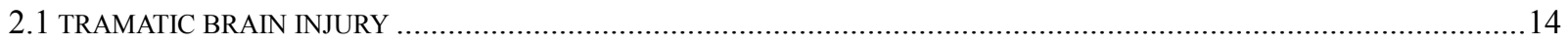

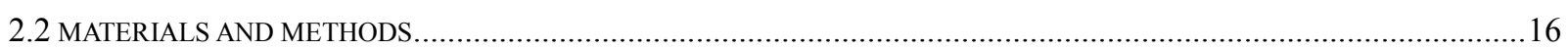

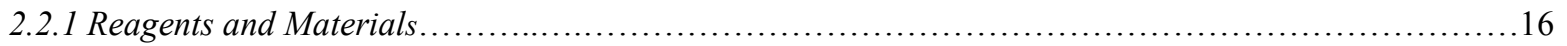

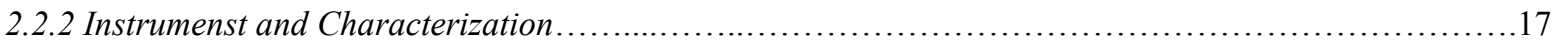

2.2.3 Synthesis of Nanoparticles and Conjugation with Detection Antibodies...............................17

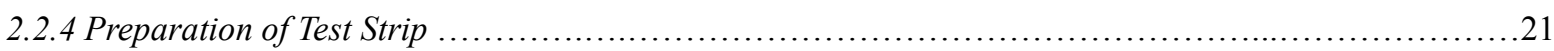

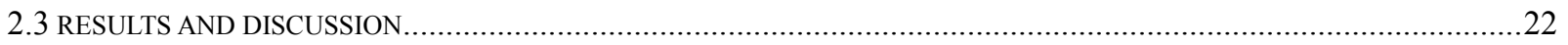

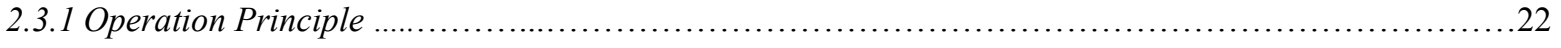

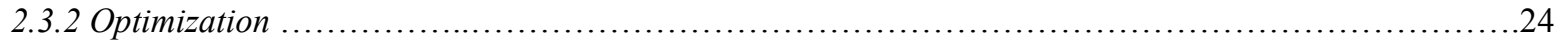

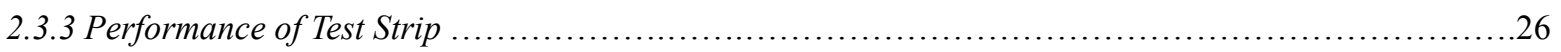

2.3.4 Measurement of Anti-interference Ability in Blood Plasma ............................................27

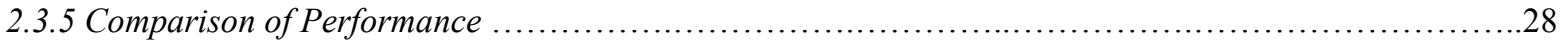

2.3.6 Measurement of Protein Biomarker in Diluted Blood Plasma .....................................29

2.3.7 Measurement of Protein Biomarker in Clinical Blood Plasma Samples ....................................32

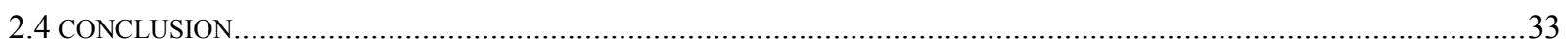

CHAPTER 3: PLASMA SEPARATION UNIT IN WHOLE BLOOD TESTING...................................................34

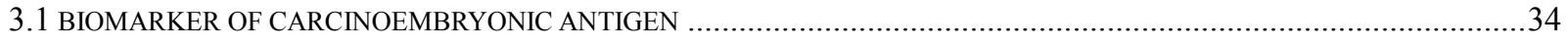

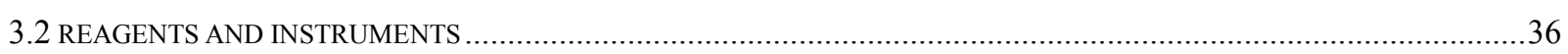

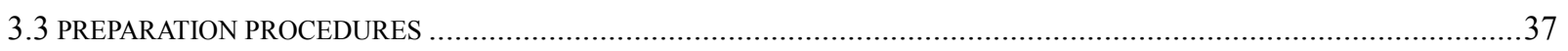

3.3.1 Preparation of of Nanoparticles..................................................................................................................3 


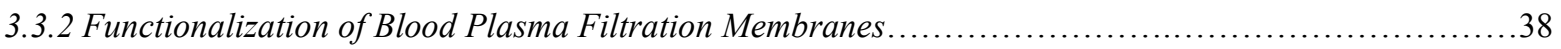

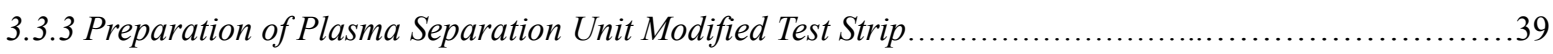

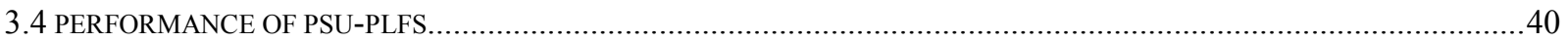

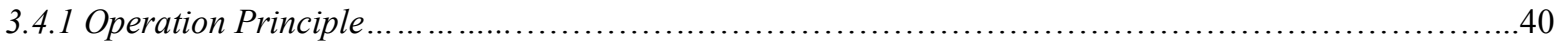

3.4.2 Apparent Separation Efficiency and Yield .......................................................

3.4.3 Analytical Characterization in Whole Human Blood ..................................................46

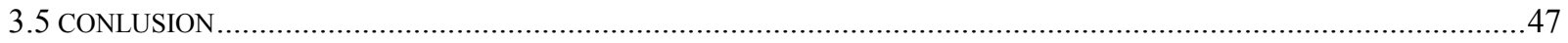

CHAPTER 4: PLASMONIC CHIP-TEST STRIP IN WHOLE BLOOD TESTING ............................................48

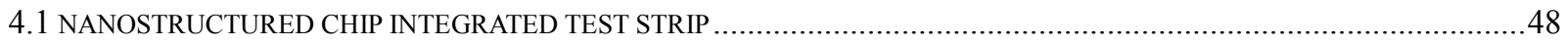

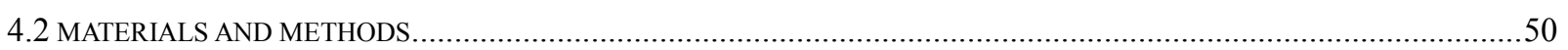

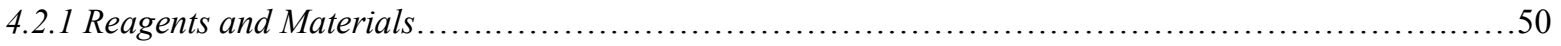

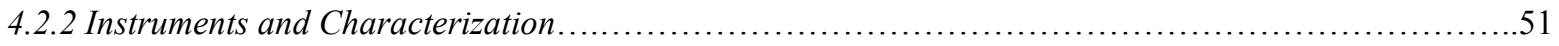

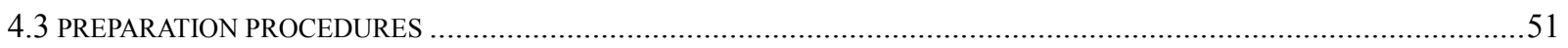

4.3.1 Synthesis of Gold Nanopyramid Array Chip and Functionalization of Capture Antibody on Chip..........51

4.3.2 Functionalization of Surfactant Bridge on Chip Surface ..............................................53

4.3.3 Synthesis of Nanoparticles and Conjugation with Detection Antibodies.................................53

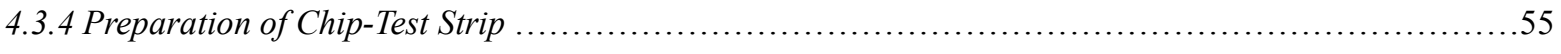

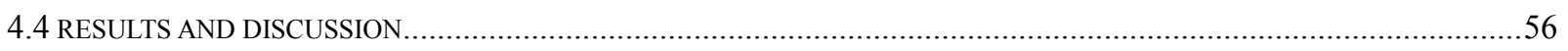

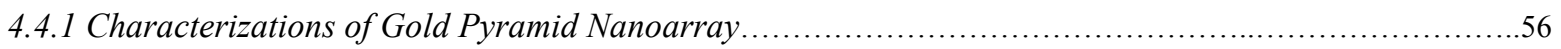

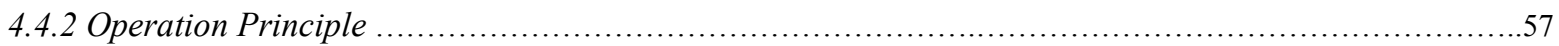

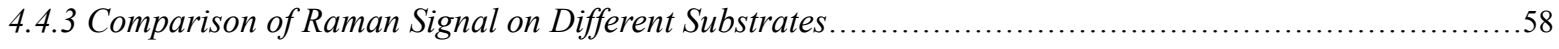

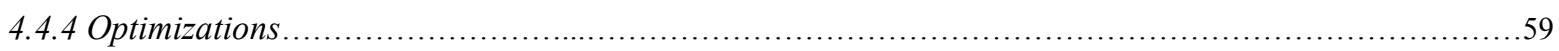

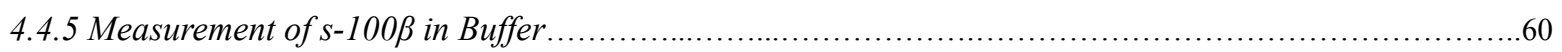

4.4.6 Measurement of s-100ß in Clinical Blood Plasma Samples....................................................61

4.4.7 Measurement of $s-100 \beta$ in Whole Blood Samples...........................................................63

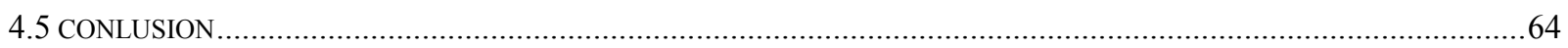

CHAPTER 5: NEAR INFRARED FLUORESCENCE IN BLOOD PLASMA TESTING ................................66

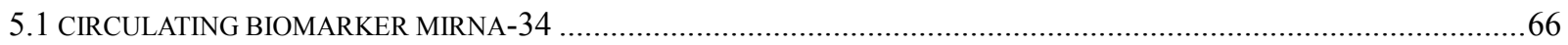

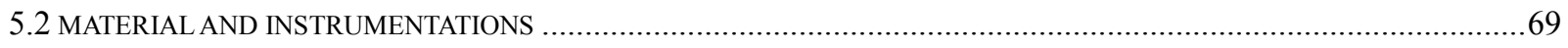

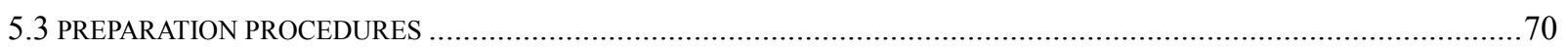

5.3.1 Preparation of Dye Encapsulated Silica Nanoparticles ..................................................... 70

5.3.2 Labeling Detection DNA with Nanoparticles................................................ 71

5.3.3 Preparation of Streptavidin-Biotinylated Capture DNA Conjugates ....................................73

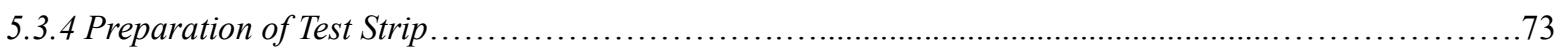

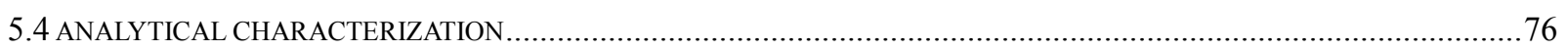

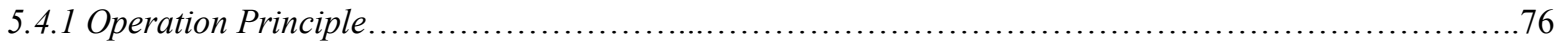




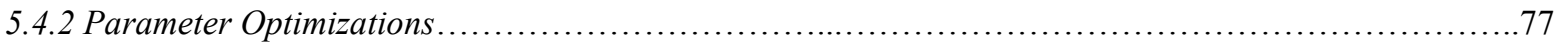

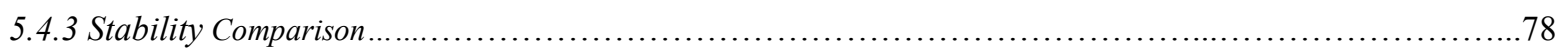

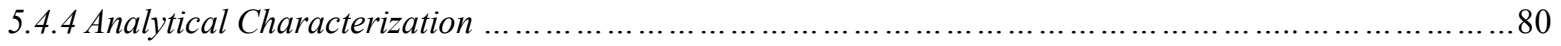

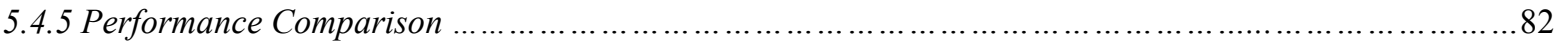

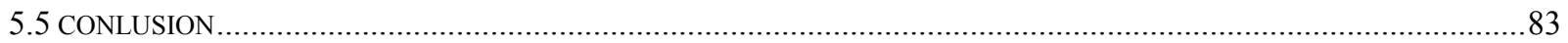

CHAPTER 6: AMPLIFICATION STRATEGY MODIFIED TEST STRIP IN PLASMA TESTING ..............85

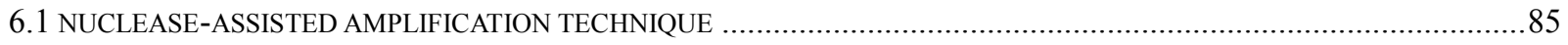

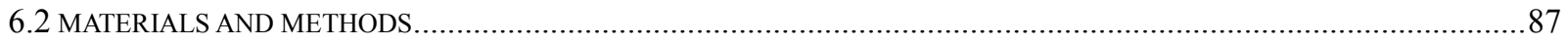

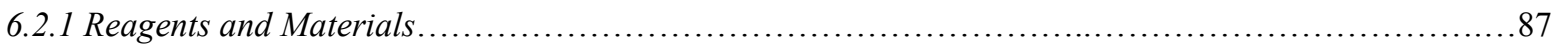

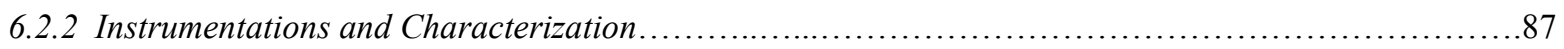

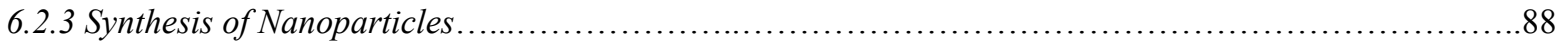

6.2.4 Labeling Detection DNA with Nanoparticless............................................... 89

6.2.5 Labeling Biotinylated Detection DNA with Streptavidin........................................ 91

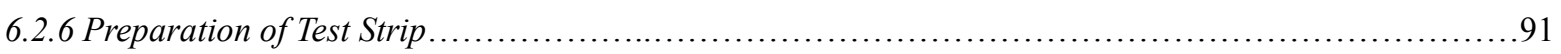

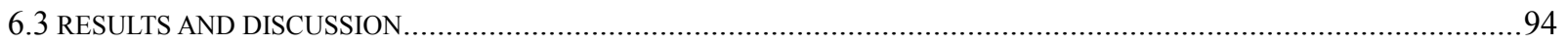

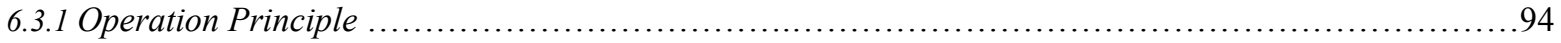

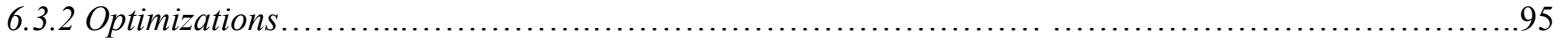

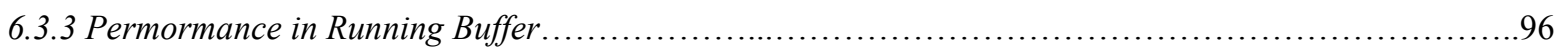

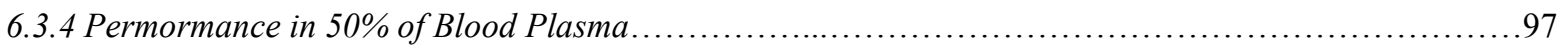

6.3.5 Measurement of miRNA-34 Biomarker in Clinical Blood Plasma Samples..............................99

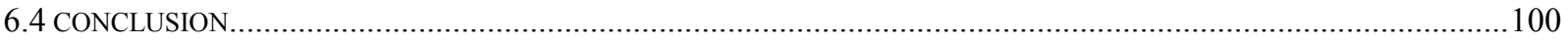

CHAPTER 7: CONCLUSION AND OUTLOOK ....................................................................................101

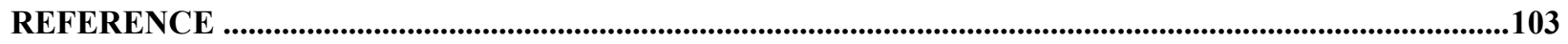

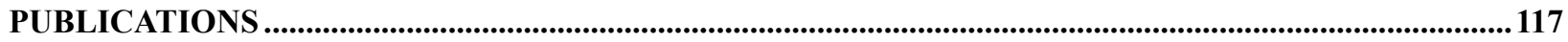




\section{Chapter 1: INTRODUCTION}

\subsection{BACKGROUND AND MOTIVATION}

A critical and challenging future direction in science and engineering is to develop technologies to improve the health of people in the developing and remote regions of the world. ${ }^{1}$ However, advanced diagnostic technologies are always centralized and requiring sophisticated instruments, specialized laboratory personnel and expensive chemicals. Moreover, continuous and long term monitoring add tremendous time and financial burden on patients due to soaring costs and frequent travel to hospitals and institutions. These factors have accelerated the emphasis on the development of low-cost, easy-to-operated and reliable devices to shift diagnostic testing from medical institutes and laboratories to the sites of patient care.

Diagnostic testing conducted at or near the patient's bedside is called point-of-care testing (POCT), which can be operated by patients themselves, caretaker or healthcare professionals outside of the clinical laboratory. In addition, POCTs allow test results to be read out facilely and quickly, which assists in making clinical management decisions in an immediate and timely manner. ${ }^{2}$ There is an increasing need of POC diagnostics, the corresponding expenses are projected to reach USD 38.13 Billion by 2022 from USD 23.71 Billion in 2017. Nowadays, the most widely applied POC device is blood glucose test strip, which was proposed in 1962 and bolstered by a portable pregnancy test strip in $1977 .{ }^{3}$ Since then, numerous paper-based lateral flow strips (PLFSs) has been developed for drugs screening, food pathogens screening and infectious disease testing. Most of the testing samples are human fluids, such as whole blood, serum, plasma, saliva and urine, which are easy to be collected and can provide diverse sensing pathways in a less-invasive mode. The underlying mechanism of a PLFS is based on sensing of a target molecule or biomarker. For example, a pregnancy test strip is manufactured for detecting human chorionic gonadotrophin (hCG) from urine sample, which is a protein biomarker relating with the pregnancy progression. ${ }^{4}$ In a typical pregnancy test, the urine sample is firstly collected into a container. And then the sample is moved from the container to the sample area of the pregnancy test strip through a dropper. By capillary force, the specimen flows from the sample area to the detection area. If $\mathrm{hCG}$ is present in the specimen, it will react with the conjugated NPs, which binds to the hCG-antibody on the detection area to generate a colored band. Presence 
of two colored bands, which are respectively located at the detection area and control area, indicating a positive result; while the absence of the band located at the detection area indicates a negative result. The test procedure can be completed generally within $20 \mathrm{~min}$. However, there are tradeoffs. In order to simplifying and reducing the cost, the sensitivity and accuracy of a PLFS are always sacrificed. As a result, most of the current PLFSs rarely deal with early-stage biomarker detection, owing to the fact that early stage diseases always relate with trace amounts of biomarkers. In addition, a relative large quantity of interference molecules existing in human fluids decreases sensing specificity and reduce signal accuracy of the devices. Therefore, it is of great significant to develop PLFSs with elevated performances to address the unmet need for sensitive and reliable measurement of early-stage biomarker from human fluids.

\subsection{OBJECTIVE}

In this dissertation, the objective is to address the problems and develop a sensitive, portable and low-cost sensor platform towards POC applications for early-stage biomarker detection in human fluids. To meet the demands, the following objectives are of paramount importance:

1) The developed sensor should be portable, easy-operated and low-cost;

2) The proposed device should address the limitations of current PLFSs, in terms of sensitivity, specificity and anti-interference ability;

3) The proposed sensor platform should show promises as a real POC tool for practical applications.

\subsection{SIGNIFICANCE}

There is an increasing need of employing POC tools for early-stage disease monitoring. However, most of the reported biosensors suffer from low sensitivity and poor anti-interference capacity towards non-specific interference biomolecules in complex human fluids, which limit their applications in early stage disease detection. Therefore, my research aims to fill the gaps and overcome those challenges to fabricate viable and portable biosensors with high sensitivity and anti-interference capability.

The proposed research is significant in that:

1) It demonstrates the limitations and gaps between the existing state-of-the-art biosensors 
and the desired POC tools;

2) It proposes innovative approaches and sensing mechanisms to address the challenges of PLFSs;

3) The proposed approaches and sensing mechanisms can be adapted to other applications, exhibiting transformative potential of the work.

As a result, the developed PLFS in this dissertation enables direct disease biomarker detection from clinical plasma samples and whole blood samples. In addition, the obtained data from the developed PLFSs was comparable with that from the clinical standard ELISA method, exhibiting great promises as potential POC tools for practical applications.

\subsection{INNOVATION}

Several innovative strategies have been proposed in this dissertation to address the issues arose from current PLFSs, including:

1) Ultrasensitive detection was realized through employment of surface-enhanced Raman scattering (SERS) technique and fluorescent technique into PLFS;

2) The interference from non-specific biomolecules from human fluids was reduced by tuning optical property of nanomaterials to the biological transparent window;

3) The anti-interference ability of the functional nanomaterials was improved through coating nanomaterials with silica layers, which can serve as a barrier to NPs from the interference biomolecules in human fluids;

4) The interference of red blood cells (RBCs) from whole blood was reduced via modifying PLFS with plasma separation unit (PSU); the separation efficiency and separation rate was substantially elevated through employing functional chemicals;

6) Signal amplification strategy of nuclease modification was introduced into PLFS for improving signal intensity and sensitivity.

The reason for choosing SERS technique and fluorescent technique to modify PLFS is owing to the fact that they can offer LFTS with substantially elevated signal intensity and detection sensitivity. Moreover, compared with SERS technique, fluorescent technique exhibits an additional advantage in simplicity during signal acquisition. 


\subsection{OPERATION PRINCIPLE OF TEST STRIP}

PLFSs have been developed for analyzing target analytes within particular fluids beginning in the late 1970s with the advantages of simplicity, low cost, portability and instantaneous diagnosis in the proximity of the patients. After that, PLFSs have been widely used in biomedical applications, environmental monitoring, food safety, and clinical diagnosis for detecting various biomolecules and chemical contaminants, including infectious agents, nucleic acids, proteins, cells (i.e. cancer cells), veterinary drugs, and toxins. The most well-known applications of PLFSs are glucose test strips and pregnancy test strips.

A general PLFS consists of a series of chromatographic pads containing dry reagents which can be activated by the fluid samples. Each chromatographic pad possesses different capillary properties and capacity in order to transport fluid smoothly and efficiently. A PLFS contains four chromatographic pads, including the sample pad, conjugate pad, nitrocellulose membrane and absorption pad. Details about each component functions as well as the assembly of the PLFS are listed below and illustrated in Figure 1.1.

Sample pad: It is used to promote and distribute the sample fluid to the conjugate pad evenly. In this dissertation, cellulose fiber $(17 \mathrm{~mm} \times 30 \mathrm{~cm}$, Millipore $)$ was employed as the sample pad.

Conjugate pad: It is made from glass fiber. The glass fiber can easily adsorb aptamer, DNA or antibody labeled nanoparticles (signal reporters). In this dissertation, glass fiber of $8 \mathrm{~mm} \times 30$ $\mathrm{cm}$ was utilized.

Nitrocellulose (NC) membrane: The most important component of the PLFS is the NC membrane. The pore size and the material properties of the membrane influence the flow rate (reaction time), the binding stability and the width of the test zone as well as the sensitivity of the entire system. In this dissertation, the $\mathrm{NC}$ membranes with the dimensions of $25 \mathrm{~mm} \times 30 \mathrm{~cm}$ and pore size of $0.22 \mu \mathrm{m}$ and $0.45 \mu \mathrm{m}$ were employed.

Absorption pad: The function of the absorption pad is to adsorb the excess fluid from the device while acting as a sink. Capillary effects draw fluid through the NC membrane from the sample pad into the absorption pad, maintaining constant motion until an equilibrium in capillary pressure is achieved. Therefore, the absorption pad enables more target analyte contained sample fluid to be extracted and detected over an increased time interval, leading to an improved sensitivity. In this dissertation, cellulose fiber with the dimensions of $17 \mathrm{~mm} \times 30 \mathrm{~cm}$ was used as absorption pad. 
Assembly of the PLFS: The sample pad, conjugate pad, NC membrane and absorption pad were laminated to a plastic adhesive backing layer $(60 \mathrm{~mm} \times 30 \mathrm{~cm}$, Millipore $)$. Each component was overlapped by $2 \mathrm{~mm}$ to ensure the migration of liquid throughout the PLFS. The mounted paperboard was cut lengthways and divided into individual PLFS with a 3-mm width by a guillotine cutter and then stored in a dry state. In a typical assay, a certain amount of sample solution is loaded onto the sample pad of a PLFS. Subsequently, the sample solution migrates by capillary action and successively passes the conjugate pad, NC membrane and finally arrived at the absorption pad.

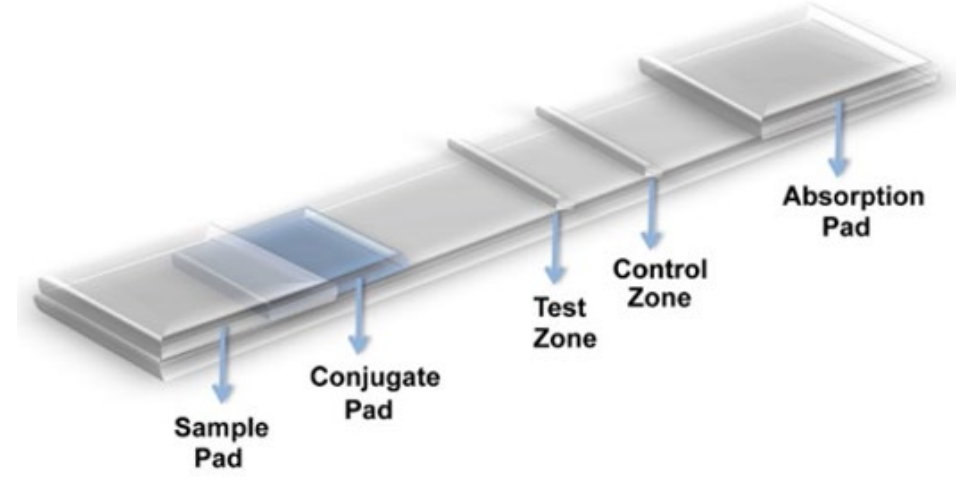

Figure 1.1. Schematic illustration of a blank PLFS.

The mechanism of a PLFS is based on the combination of signal reporting and interfacing biological recognition events between biorecognition molecules, such as the interaction between an antibody with an antigen. On conjugate pad, detection reagents are immobilized, which contains detection biomolecules labeled signal reporters. On test zone and control zone, capture reagents and control reagents are respectively functionalized. There are two most frequently employed interaction formats for capturing target analyte on the detection zone of a PLFS: competitive format assay and sandwich format assay. Their corresponding response is inversely proportional to and proportional to the concentration of the analyte respectively. The competitive format works best for the target molecules with low molecular weight or presenting single specific antigen. The competitive format contains two sensing mechanism: 1) antibody coated format (Figure 1.2b): the competition between target antigen and signal reporter linked antigen. In this format, the specific antibody is firstly immobilized on the test zone. During the detection, the target antigen and signal reporter labeled antigen would compete for binding on the specific antibody on the test zone; 2) antigen coated format: the competition between antibody/signal 
reporter and the complex of antigen-antibody/signal reporter (Figure 1.2c). The target analyte contained sample solution is firstly incubated with the specific antibody/signal reporter to form the antigen-antibody/signal reporter. The resulting complex would compete with antibody/signal reporter for binding on the specific antigen immobilized test zone. Besides the above mentioned competitive format, another format is sandwich structure based format. In this format of assay, detection antibody is firstly functionalized with signal reporters; while the capture antibody is immobilized on the test zone. As shown in Figure 1.2a, during the detection, a sandwich structure of antibody-antigen-detection antibody/signal reporter is formed on the test zone of the PLFS. On the control zone of the PLFS, it is immobilized with secondary antibodies, which is employed for capturing the excess detection reagents from the test zone. The control line is utilized to indicate that the test strip functions properly.

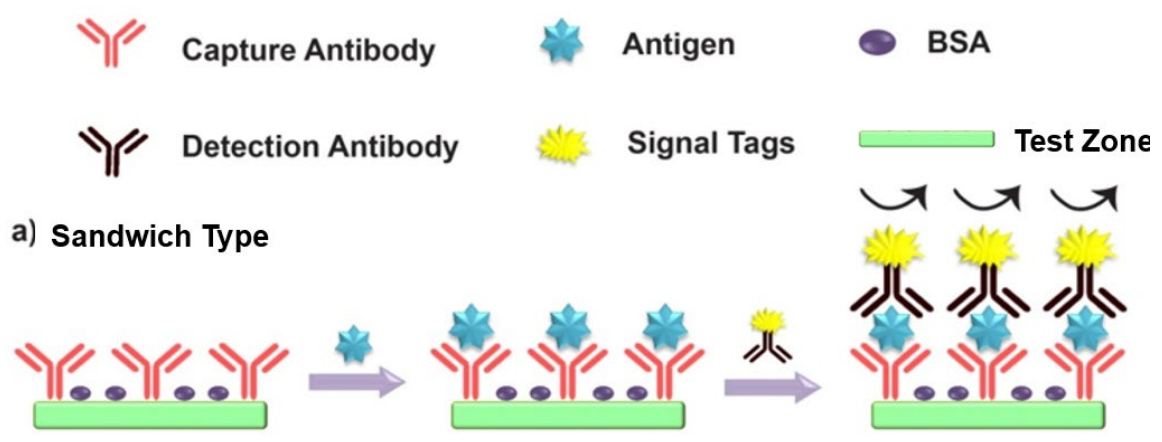

b) Competition Type-antibody coating

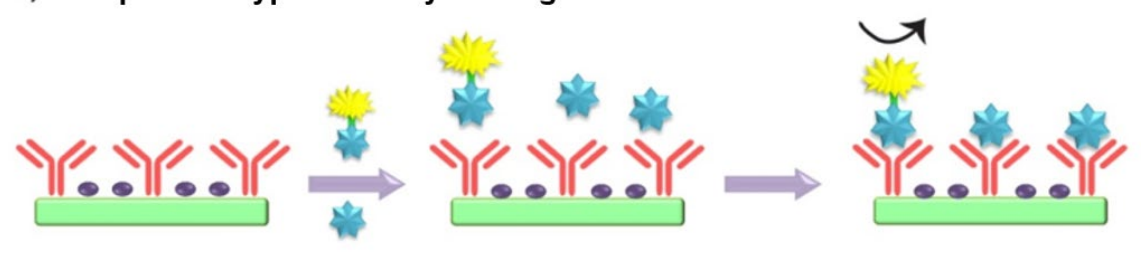

c) Competition Type-antigen coating

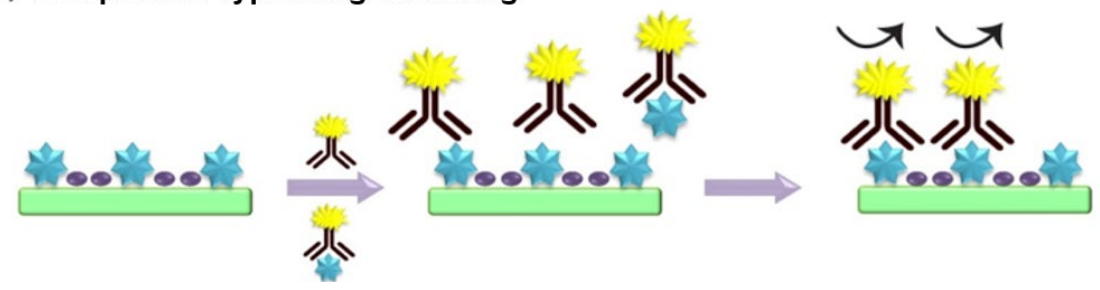

Figure 1.2. Schematic illustration of the operation principle of sandwich format assay (a) and competitive format assay with antibody coating type (b) and with antigen coating type (c). 


\subsection{STRATEGIES FOR IMPROVING TEST STRIP PERFORMANCES}

Nanomaterials possess unique properties, such as large surface area, excellent biocompatibility, outstanding conductivity and catalytic ability. Employment of nanomaterials exhibits great promise in elevating sensor performances. For example, the electron transfer rate can be elevated from 700 to 5,000 when gold nanoparticles (GNPs) were immobilized into a biosensor. ${ }^{5}$ In addition, in an enzyme labeled biosensor, nanomaterial modifications can offer diverse microenvironment and more freedom of orientation for enzymes. ${ }^{6}$ Moreover, the stability of enzymes can be improved when enzymes were linked with GNPs. ${ }^{7}$ The properties of nanoparticles strongly depend on their size and morphology. ${ }^{8}$ For example, in aqueous solution, spherical GNPs with an average diameter of $20 \mathrm{~nm}$ exhibit strong absorption at $\sim 520 \mathrm{~nm}$ in wavelength, which makes the GNPs present red color. While the GNPs start to aggregate, the absorption peak shifts to longer wavelength and the color of the GNPs changes to blue correspondingly. This unique property brings about many developments of binding-induced aggregation sensors. ${ }^{9}$ In a PLFS, GNPs are the most extensively employed signal reporters owing to their inherent advantages, including intense optical properties, vivid color and biocompatibility. In addition, the optical properties, size and morphologies of GNPs can be simply adjusted by changing the ratio between the reductant (sodium citrate) with $\mathrm{HAuCl}_{4}$, offering PLFS with diverse designs and developments for different sensing demands. ${ }^{10}$ The detection sensitivity of GNPs tagged PLFSs can be further elevated through nanoparticlepromoted precipitation of gold or silver around GNP tags. Such precipitation enlarged the size of the NP-tags and enhanced the optical intensity of the test line, which amplifies the events of biorecognition, meanwhile benefits the colorimetric signal readout, leading to an elevation in detection sensitivity. ${ }^{11}$ Another effective approach for improving the GNP-PLFS sensitivity is to co-label enzyme onto GNP surfaces and then introducing enzyme substrates for amplifying signals. The most commonly used enzyme and enzyme substrate in PLFS is horseradish peroxidase (HRP) and TMB/ $\mathrm{H}_{2} \mathrm{O}_{2}$. In a typical assay, after the HRP labeled GNPs are captured on the test area, the substrate $\mathrm{TMB} / \mathrm{H}_{2} \mathrm{O}_{2}$ is introduced to improve the test line intensity. Compared with the conventional PLFS, the enzyme functionalized PLFS can realize one to two orders of magnitude improvement in detection sensitivity. ${ }^{12}$ Besides HRP, glucose oxidase and alkaline phosphatase are also the good choices for enzyme catalytic amplification. Polymerase chain reaction (PCR) is an effective approach for amplifying DNA concentrations with thousands 
to millions of more copies of that particular DNA segment. Modifying and integrating the polymerase reactions into PLFSs can significantly improve the detection sensitivity. A new strategy called strategy isothermal strand-displacement polymerase reactions (ISDPR) was developed and integrated into PLFSs to overcome the disadvantages of PCR. Compared with conventional PCR, no thermal cycling is required in the ISDPR-PLFS. In addition, the amplification procedure was significantly shortened to less than half an hour. ${ }^{13}$

Besides GNPs, magnetic nanoparticles, microparticles, fluorescent dyes and quantum dots have also been employed into PLFSs as signal tags. The use of magnetic beads can offer the PLFSs with improved sensitivity. Since the noise from nonspecific adsorption was reduced by the magnetic separation procedure, while the signal was elevated owing to the large surface area of magnetic beads. Fluorescence techniques have been extensively applied in biological sensing on PLFS. Their performances are largely dependent on the physicochemical properties of the employed fluorophores. Several experimental parameters should be taken into account when choosing a fluorophore, including excitation and emission spectra, fluorescence intensity, lifetime and emission anisotropy. Organic dyes are often used as the fluorophores in biological research owing to their small sizes, which are suitable for conjugation with biomolecules. Additionally, various dyes with characteristic excitation and emission spectra are commercially available and the corresponding protocol for functionalization is well established. However, the photobleaching, narrow excitation spectra and low luminescent restrict their further applications.

The employment of quantum dots (QDs) into fluorescent biosensors can address these challenges and meet the demands of fluorescent research owing to their unique properties, including resistant to photobleaching (fluorescence lifetimes: 1-10 ns vs 10-100 ns), sizedependent emission, narrow emission bandwidths and high levels of brightness. ${ }^{14}$ For example, the values of molar absorption coefficients at the first absorption band of QDs are generally larger than the organic dyes $\left(100,000-1,000,000 \mathrm{M}^{-1} \mathrm{~cm}^{-1}\right.$ vs 25,000-250,000 $\left.\mathrm{M}^{-1} \mathrm{~cm}^{-1}\right)$; while the quantum yields are $0.1-0.8$ (visible), 0.2-0.7 (near-infrared (NIR)) from QDs and 0.5-1.0 (visible), 0.05-0.25 (NIR) from organic dyes. Additionally, with the light illumination, QDs can emit light of specific frequencies. The emission frequencies can be precisely tuned by changing the sizes and components of QDs, which offers QDs the flexibility for multiple detections with different emission peak positions. ${ }^{15}$ Lin's group reported a QDs based fluorescent PLFS for rapid (10 min) detection of protein in both aqueous solutions and spiked human plasma samples. ${ }^{16}$ The 
quantification for target protein was based on recording the fluorescent intensity of QDs that were captured on the test zone of PLFS through the specific biorecognition between antigen and antibody. LODs for target protein of 1 and $8 \mathrm{ng} / \mathrm{mL}$ were obtained from aqueous solutions and spiked human plasma samples respectively. This QDs based fluorescent test strip can be extended for the fast and simple analysis of other protein biomarkers.

Recently, NIR nanomaterials attract increasing attention in bioassays due to that the biological tissues are highly transparent to the NIR light (700 to $1,100 \mathrm{~nm})$. Studies showed that various nanomaterials present optical absorbance in this special NIR spectral window, such as NIR-QDs, SWNTs, ${ }^{17}$ graphene dots, ${ }^{18}$ and upconversion-luminescent phosphors (UCPs). ${ }^{19}$ Among the NIR NPs, QDs exhibit flexibility for multiple detections; while carbon nanomaterials and UCPs show low toxicity to biological samples, which are well suited for biomolecule analysis. As a result, the employment of NIR into PLFS shows an increase in sensitivity and anti-interference capacity in biological fluids compared with that of the conventional GNP-PLFSs.

Signal quantity system is also crucial for improving PLFS performances, owing to the fact that proper signal recording approach can avoid signal loss, meanwhile generate stable signal responses, leading to the elevation of sensitivity and stability of a biosensor. In general, signal recording system is selected according to the employed signal reporters. For example, a colorimetric strip reader is designed for recording the color intensity from the test line of a GNP labeled PLFS; while a fluorescent strip reader is designed for recording the fluorescent signal from the test line of a QDs, fluorescent dye or UCPs functionalized PLFS. Recently, a concept of thermal contrast was proposed into the PLFS as the signal quantity system for improving the signal intensity and detection sensitivity. ${ }^{20}$ A laser/light emitting diode (LED) and an infrared temperature gun were employed instead of the conventional strip reader as the signal quantity system. The light was used to irradiating the GNPs captured on the test line while the infrared temperature gun was employed to record the thermal contract from the heated GNPs. As a result, this thermal contract mechanism endowed the GNP-PLFS with an improvement of 32-fold in detection sensitivity. The performance can be further improved through the employments of higher-absorbing NPs and low-absorbing PLFS backing materials. ${ }^{21}$

The capacity of simultaneous detection of multiple biomarkers is another important factor to elevate the sensor performances, since measurement of multiple biomarkers is considered as the most promising approach to reliable diagnosis or prediction of diseases. For example, the 
detection sensitivity for ovarian cancer was improved from $65 \%$ to $74 \%$ and the detection specificity was elevated significantly from $52 \%$ to $94 \%$ when multiple protein biomarkers were analyzed. ${ }^{22}$ Moreover, compared with single detection, simultaneous detection offers additional advantages, including timesaving, reduced operations, cost and samples. ${ }^{23}$ Simultaneous detection in PLFS can be realized through dispensing capture reagents on different test line respectively; meanwhile functionalizing signal tags with different detection reagents towards specific target biomolecules. In general, four test-lines is the maximum allowable dispensed amount on a PLFS according to the chemical reagent consumptions of a PLFS with general dimensions $(3 \mathrm{~mm} \times 60 \mathrm{~mm})$. The signal tags functionalized detection reagents can be either mixed and then loaded on one conjugate pad of a PLFS, or loaded onto different conjugate pads of the multiple conjugate pads modified PLFS.

In summary, the major advantages of PLFSs are portable, fast, low-cost and easy-operated. However, most of the current state-of-the-art PLFSs can only function in buffers or artificial solutions. It is still a formidable challenge to apply them in real applications owing to the relative low sensitivity, which cannot meet the demands for detecting early stage of disease biomarkers. Additionally, the high interference of nonspecific biomolecules in complex human fluids dramatically affects the sensing specificity and stability. By taking the advantages of explosive development of nanotechnology, many advanced techniques and strategies can be employed to solve the problems.

\subsection{TECHNIQUE OF SURFACE-ENHANCED RAMAN SCATTERING}

Raman spectroscopy has been intensively explored in the applications of biological sensing and medical diagnostics. However, the Raman scattering is much weaker than the other optical techniques, which is considered to be the main hurdle for ultrasensitive measurement for relative low sample concentrations.

Metallic nanomaterials possess unique optical properties that are related to the collective oscillation of free conduction electrons in nanostructures in resonance with the incident electromagnetic radiation. These nanostructured materials are capable of providing surface plasmon resonances (SPRs) and can be utilized in biosensing technologies based on the principle that the intensity and the position of SPR is sensitive to the changes in the dielectric properties (refractive index) in the vicinity of the plasmonic surfaces. SPR occurs in two different forms: 
propagating surface plasmon polaritons (SPPs) and localized SPR (LSPR). SPPs are the charge oscillations associated with an electric field propagating along a metal-dielectric interface. These oscillations are decaying exponentially in the perpendicular direction. LSPR occurs when the dimensions of a metallic nanostructure are much smaller than the incident wavelength, leading to non-propagating local oscillations of the plasmons around the nanoparticles. By utilizing plasmonic materials, numerous nanostructures have been developed as signal amplifiers for sensitive optical sensing. Surface-enhanced Raman scattering (SERS) is a powerful technique that possesses ultra-sensitivity by amplifying the Raman scattering signal by orders of magnitude if a molecule is associated to the surface of plasmonic nanomaterials. ${ }^{24}$ The most widely employed SERS-active metals are $\mathrm{Au}, \mathrm{Ag}$ and $\mathrm{Cu}$. The Raman signal enhancement of SERS is attributed to two mechanisms: chemical enhancement and electromagnetic (EM) enhancement. The chemical enhancement (10-100 enhancement factor) contributes to the electron interaction between the metallic substrate and the molecule absorbed on its surface; while the electromagnetic enhancement $\left(10^{4}\right.$ and $10^{12}$ enhancement factor) induces the dominant contribution and it arises from the light-induced collective oscillation of conduction electrons from the metallic substrate. ${ }^{25}$ During developing of a biosensor, the SERS-active nanomaterials can be integrated as labels or patterned substrates.

"Hot spots" are generated inside the gaps or around the sharp edges, corners or tips of the SERS-active nanomaterials, where the EM field is particularly intense, leading to extraordinary enhancement of SERS signal. Nowadays, a SERS enhancement factor of $10^{14}$ have been achieved through precisely fabricating the nanostructure, which is capable of sensing single molecule. ${ }^{26}$ SERS is also well-suited for multiple detections because of the "fingerprint" feature to produce distinct spectra from components in multiplexing. On the other side, this "fingerprint" ability can also increase the anti-interference resistance to nonspecific molecule in complex sample matrices. Therefore, SERS technique is well-suited to be used for ultrasensitive measurement in biological matrices. 


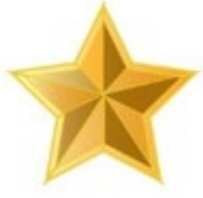

Au Nanostar

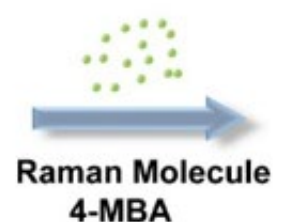

4-MBA
4-MBA Adsorbed

Au Nanostar

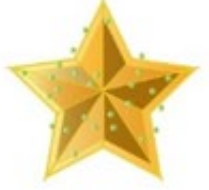

\section{TEOS \\ Ammonium}

\section{SERS Probe Au Nanostar@MBA@Silica}

Figure 1.3. Structure of sandwich typed SERS probe with $\mathrm{SiO}_{2}$ coating.

Conventional SERS biosensors are developed using bare GNPs, which supports localized surface plasmon resonance (LSPR) for the absorbed Raman molecule to generating SERS signals. However, bare GNPs-based SERS probes are unstable in aqueous solutions with high ionic strength or in blood samples, which raises serious concern about reliability and robustness. In addition, the SERS labels are easy to desorb from the nanoparticle surface, leading to reductions of stability and signal intensity during the detection. A solution to this issue is to coat the SERS labels adsorbed nanomaterials with a thin protecting layer, such as polyethylene glycol (PEG) or $\mathrm{SiO}_{2}$ to form a sandwich-typed SERS label. The protective layer has several advantages: (i) it encapsulates numerous Raman reporters into a single particle, which enhances the SERS response; (ii) the protecting layer avoids the leaking out of Raman reporters, which increases the signal stability; (iii) it offers the capability for further surface-functionalize with biomolecules; (vi) it makes the nanoparticles water-soluble and biocompatible. In this dissertation, Au nanostar will be employed as the SERS substrate for the absorbed Raman molecule to generating a strong plasmonic field foe amplifying SERS signal. While the Raman reporter molecules are sandwiched between an $\mathrm{Au}$ nanostar and a thin silica shell. This structure prevents Raman reporter molecules from leaking out. The Au nanostar generates a strong plasmonic field for amplifying SERS signal. The silica shell not only provides a platform for bioconjugation but also makes the SERS probe water-soluble. The structure of the sandwich typed SERS probe is illustrated in Figure 1.3.

\subsection{TECHNIQUE OF NEAR-INFRARED FLUORESCENCE (NIRF)}

Fluorescence techniques have been extensively applied in biological sensing, cell imaging and screening. Their performances are largely dependent on the physicochemical properties of the 
employed fluorophores. Several experimental parameters should be taken into account when choosing a fluorophore, including excitation and emission spectra, fluorescence intensity, lifetime and emission anisotropy. ${ }^{27}$ Organic dyes are often used as the fluorophores in biological research owing to their small sizes, which are suitable for conjugation with biomolecules. Additionally, various dyes with characteristic excitation and emission spectra are commercially available and the corresponding protocol for functionalization is well established. However, the photobleaching, narrow excitation spectra and low luminescent restrict their further applications. $^{28}$

The employment of near-infrared fluorescence (NIRF) attracts increasing attention in biological applications owing to that it can reduce the damage of biological samples and avoid the photobleaching and background interference when visible fluorophores (VisF) are utilized. ${ }^{29}$ This is because that due to reduced absorbance and minimal scattering, the NIR radiation possesses high penetration ability towards tissues and biological matrices. Meanwhile, the NIR wavelengths falls within the biological windows with low background fluorescence signals, which provides elevated signal-to-noise ratio. ${ }^{30}$ In this dissertation, NIRF dye-encapsulated silica nanoparticles have been developed. The superior properties of such nanoparticles include amplified signal, outstanding photostability, water-solubility and viability of surface modification. For example, the silica layer enables a large number of NIRF dye molecules to be encapsulated inside of one single silica nanoparticle, which can dramatically magnify its luminescence signal. In addition, the external silica layer protects the dyes from the surrounding matrix or other environment, which minimizes the photobleaching phenomena and increases its photostability. Moreover, silica possesses flexible routes for surface modifications with various types of functional groups (e.g. $\left.-\mathrm{NH}_{2},-\mathrm{COOH},-\mathrm{SH}\right)$, which allows its conjugation with different biomolecules during bioassays. ${ }^{31}$ 


\section{Chapter 2: SURFACE ENHANCED RAMAN SCATTERING TEST STRIP FOR PLASMA TESTING}

\subsection{TRMATIC BRAIN INJURY}

Traumatic brain injury (TBI) is a leading cause of death and disability in both the United States and the developed world. Approximately 2.8 million TBI-related emergency department (ED) visits, hospitalizations and deaths occurred in the United States in 2013. The estimated direct and indirect medical costs of TBI were estimated to be in excess of \$76 billion in 2010 . The current ED standard of care for the initial assessment of a patient's potential TBI includes employment of the Glasgow Coma Scale (GCS), in which a clinician numerically assesses the brain impairment based on the bedside physical examination, and computed tomography (CT) scans. These screening tools are presently used for initial clinical management and prognosis of TBI. ${ }^{32}$ However, they cannot provide information on pathophysiological processes, and they are not very sensitive or specific, particularly in the case of mild or moderate TBI. On the other hand, some ED patients with traumatic injuries and a poor GCS are subject to brain impairment due to intoxication from alcohol and/or drugs instead of from a true brain injury. Although early CT scans are useful for detection of intracerebral hemorrhage, they are often less effective at early detection of the more common cerebral contusions and diffuse axonal injury. Additionally, there has been a growing concern regarding radiation exposure from the use of screening CT scans in patients with possible TBI. Magnetic resonance imaging (MRI) is much more sensitive for detecting early TBI and does not expose patients to radiation. However, these studies are expensive and time-consuming, often require patient sedation, and are frequently unavailable at the ED. ${ }^{33}$ Early and accurate diagnosis of TBI is clinically important, as currently routine yet potentially unnecessary screening imaging might be avoided. Additionally, many patients with even mild TBI may experience short- or longterm sequelae including changes in attention and memory and emotional disturbances that may benefit from early diagnosis and intervention. Importantly, TBI clinical trials such as those testing potential early therapies aimed at, for example, decreasing secondary injury have been severely hampered by the dilemma of early diagnosis of true TBI as opposed to intoxication, with this "noise" requiring an increase in sample size and expense, and possible dilution of the measurable effect of the potential therapy. 
Measurement of protein biomarkers can provide an alternative route for rapid and accurate disease diagnosis. Protein biomarkers are present in human fluids, such as blood, saliva, or urine, which makes the tests much less invasive, rapid, and inexpensive and avoids the radiation involved with CT scans and time, sedation, and expense of MRIs.

The most common TBI biomarkers are glial fibrillary acidic protein (GFAP), S100- $\beta$, and neuron-specific enolase (NSE), which are released into circulation after the brain damage occurs. ${ }^{34}$ Several methods have been employed to measure the protein biomarkers, including enzyme-linked immunosorbent assay (ELISA), Western blot, and agarose. However, those methods generally involve time-consuming operation processes and large sample volume and require expensive instruments, which cannot be used as the point-of-care (POC) analytical tools for rapid and low-cost clinical diagnosis. Therefore, great efforts have been devoted to developing portable biosensors toward POC testing. Recently, several biosensors have been employed for NSE biomarker detection, including electrochemistry, ${ }^{35}$ chemiluminescence, ${ }^{36}$ and field-effect transistor. ${ }^{37}$ However, most of these biosensors were tested in buffer solutions and did not demonstrate the direct detection of NSE biomarker in human fluidic samples. Moreover, many biosensors still need multiple steps of operation in vials, which are not user-friendly. Hence, it remains a challenge for those sensors to be used in a POC setting.

PLFSs have received increasing attention as POC tools because they are inexpensive, userfriendly, and easy to operate. ${ }^{38}$ Colorimetric, ${ }^{12}$ fluorescent, ${ }^{16}$ and electrochemical ${ }^{39}$ transducers, are commonly used in PLFS. Unfortunately, they usually suffer from poor sensitivity and serious interference from sample matrices when they are used in blood plasma samples. In contrast, surface enhanced Raman scattering (SERS) has strong anti-interference ability in real-world samples because the wavelengths of both the excitation light and the scattering Raman signals can be tailored into the near-infrared biological transparency window, and Raman spectra are characteristic of fingerprint signatures of target molecules. ${ }^{40}$ Several papers have recently reported the incorporation of SERS transducers into PLFS for detection of proteins and nucleic acids in water or buffer solutions. In these reported SERS-PLFS, bare Au nanoparticles were used as the SERS substrates. ${ }^{41}$ Bare Au nanoparticle-based SERS probes are unstable in aqueous solutions with high ionic strength or in blood samples, which raises serious concern about reliability and robustness. ${ }^{42}$ Hence, the SERS-PLFS based on bare Au nanoparticle have not been applied to blood samples yet. To address the instability issue of bare gold nanospherebased 
SERS probes, Au nanostar@Raman Reporter@silica sandwich nanoparticles have been developed as the SERS probes in our research group, in which the Raman reporter molecules are sandwiched between an Au nanostar and a thin silica shell. This structure prevents Raman reporter molecules from leaking out. The Au nanostar generates a strong plasmonic field for amplifying SERS signal. The silica shell not only provides a platform for bioconjugation but also makes the SERS probe water-soluble. Our previous study has shown that these types of sandwich SERS probes are very stable in aqueous solutions with high ionic strength while bare $\mathrm{Au}$ nanoparticles tend to aggregate under the same condition. In the present work, the sandwich SERS probes are incorporated into a PLFS to detect the NSE biomarker in blood plasma. By comparison, a colorimetric PLFS with bare plasmonic Au nanospheres is also used for protein detection. Compared to the colorimetric PLFS, the SERS-based PLFS shows much higher sensitivity in blood plasma samples. To the best of our knowledge, this report for the first time demonstrates the successful detection of protein biomarkers in clinical blood plasma samples using a SERS-based PLFS without any purification or separation procedure. The results show a great potential of SERS-PLFS in the POC setting.

\subsection{MATERIAL AND METHODS}

\subsubsection{Reagents and Materials}

Malachite green isothiocyanate (MGITC) was purchased from Molecular Probes, Inc. 4Mercaptobenzoic acid (technical grade, 90\%) (4-MBA) was purchased from Sigma-Aldrich. 3Triethoxylsilylpropyl succinic anhydride (TEPSA) was obtained from Gelest Inc. Nhydroxysuccinimide (NHS), 1-ethyl-3-(3-(dimethylamino)- propyl) carbodiimide (EDC), and tetraethyl orthosilicate (TEOS) were purchased from Sigma-Aldrich; $\mathrm{HAuCl}_{4}$, sucrose, $\mathrm{Na}_{3} \mathrm{PO}_{4} \cdot 12 \mathrm{H}_{2} \mathrm{O}$, Tween 20, Triton $\mathrm{X}-100$, trisodium citrate, PBS $(\mathrm{pH}=7.4)$, BSA (bovine serum albumin) and sodium chloride were acquired from Sigma-Aldrich and used without further purification. Sucrose was purchased from VWR. Goat antihuman IgG polyclonal antibody, mice

antigoat $\mathrm{IgG}$ polyclonal antibody, IgG from human serum, Neuron-specific enolase from human brain (NSE), and antineuron-specific enolase antibody were procured from Sigma-Aldrich. Neuronspecific Enolase Quantikine ELISA Kits were purchased from R\&D System and Eagle Bio. The blood plasma was purchased from US Biological Life Science (P4252-56 Plasma, Human, IgG Free). Glass fibers (GFCP000800), cellulose fiber sample pads (CFSP001700), 
laminated cards with Hi Flow plus cards (HF090MC100 with RK12312 Membrane $(0.45 \mu \mathrm{m})$ ), and nitrocellulose membrane (RK13464 $(0.22 \mu \mathrm{m})$ ) were purchased from Millipore (Billerica, MA). Clinical blood plasma samples were collected from TBI patients presenting to the ED as part of the Resuscitative Endocrinology: Single-dose Clinical Uses for Estrogen pilot clinical trial for traumatic brain injury (RESCUE-TBI) conducted by researchers at the University of Texas Southwestern Medical Center and Parkland Hospital. As part of this study, the clinical samples were serially collected at various time points over the first 5 days following TBI and stored at $-80{ }^{\circ} \mathrm{C}$ prior to measurement. All work conducted in the pilot clinical trial was approved by the National Heart, Lung, and Blood Institute's Resuscitation Outcomes Consortium Protocol Review Committee and Data Safety Monitoring Board, the U.S. Food and Drug Administration, and the University of Texas Southwestern Medical Center Institutional Review Board. Use of these deidentified human samples at West Virginia University was approved by the West Virginia University Institutional Review Board.

\subsubsection{Instruments and Characterization}

All Raman spectra were acquired with a portable B\&W Tek i-Raman Plus. Fourier transform infrared (FT-IR) spectra were obtained under the attenuated total reflection (ATR) mode with Thermo Nicolet 6700 spectrometer. The nanoparticles were characterized with a transmission electron microscope (TEM, JEOL JEM-2100F) at an acceleration voltage of $200 \mathrm{kV}$. UV-visible absorption spectra were obtained with a Shimadzu UV-2550 spectrometer.

\subsubsection{Synthesis of Nanoparticles and Conjugation with Detection Antibodies}

The Au nanostars, the SERS probes, and the nanoparticles conjugated with detection antibodies were synthesized according to our previous paper. ${ }^{40}$ The preparation procedure is illustrated in Figure 2.1. Briefly, the Au nanostar@Raman molecule@Silica sandwich SERS probes were synthesized by adding $1.0 \mu \mathrm{M}$ Raman molecules into the Au nanostar suspension. Then, $200 \mu \mathrm{L}$ of $1 \%$ TEOS and $15 \mu \mathrm{L}$ of ammonium solution were added into the mixture, successively. After the SERS probes were formed, the resulting solutions were centrifuged and resolved in $500 \mu \mathrm{L}$ of ethanoic solution. The Au nanostars were coated with a 2-3 nm thick silica layer on the outer surface. Figure 2.2 shows the TEM images of the sandwichtyped SERS nanoparticles. 


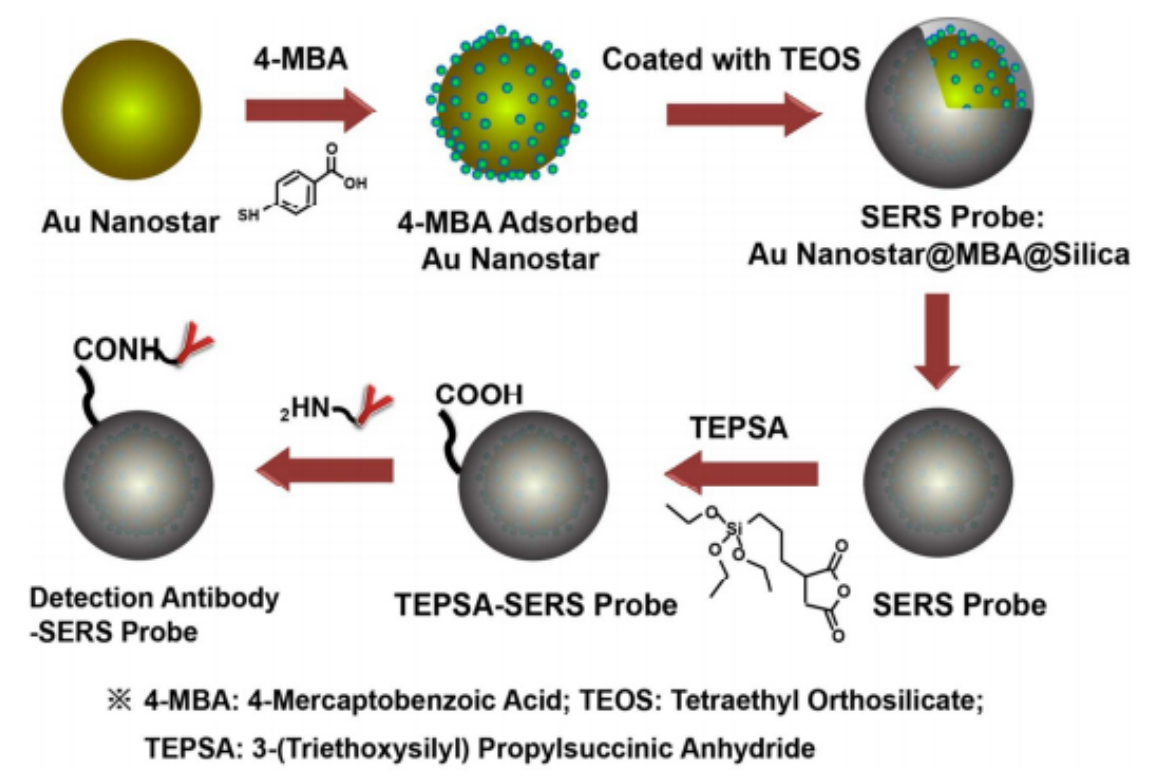

Figure 2.1. Schematic illustration of the preparation process of the SERS probe conjugated with the detection antibody.
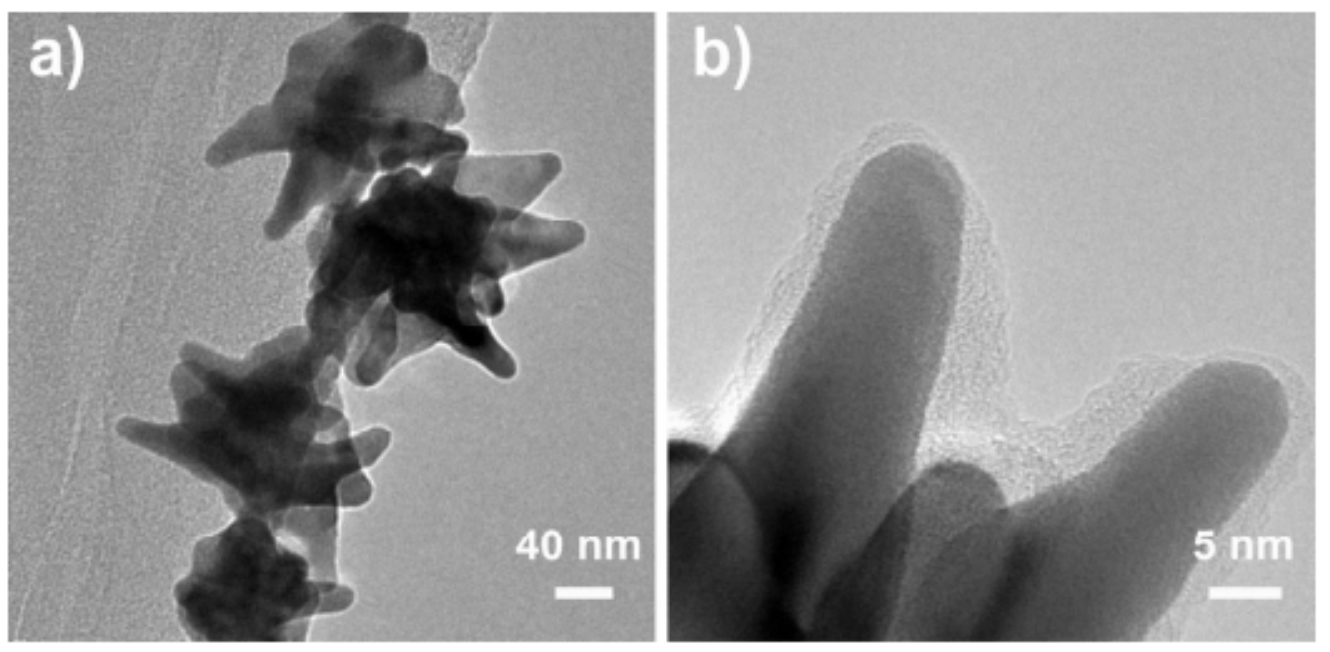

Figure 2.2. TEM images of the Au nanostar@MBA@SiO2 sandwich nanoparticles a) low magnification and b) high magnification. 


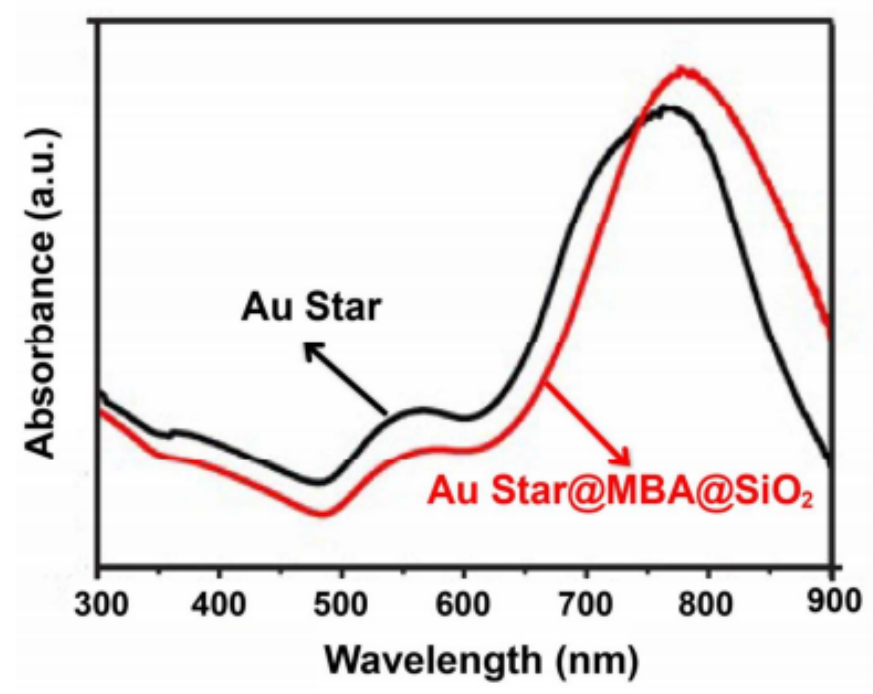

Figure 2.3. UV-visible absorption spectra of the Au nanostar and the Au nanostar@Raman molecule@silica sandwich nanoparticles.

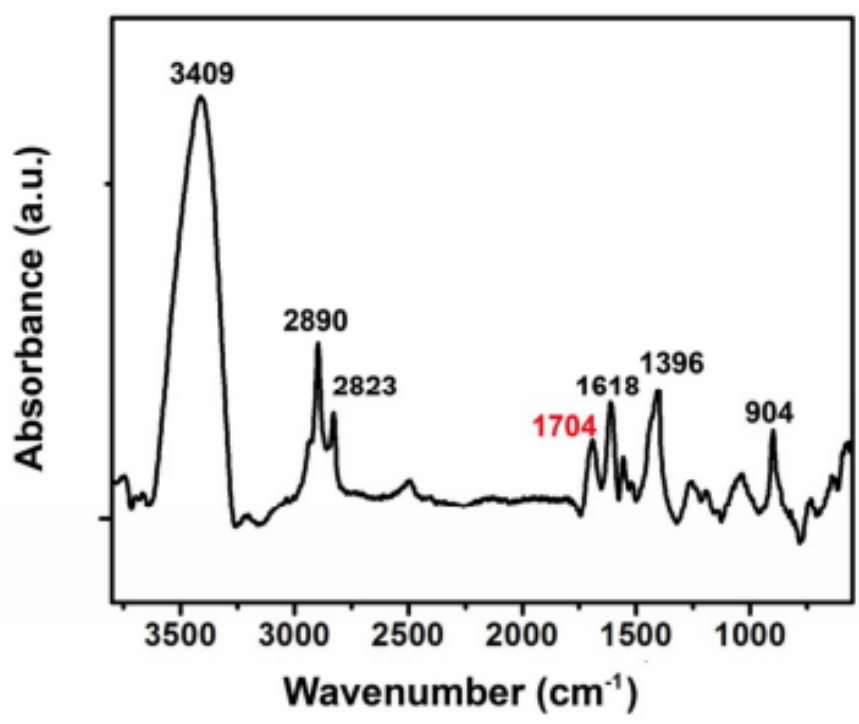

Figure 2.4. FT-IR spectra of the carboxyl-group functionalized SERS probe. The peak at 1704 $\mathrm{cm}^{-1}$ peak is assigned to the $\mathrm{C}=\mathrm{O}$ stretching vibration in $\mathrm{COOH}$ of TEPSA. 


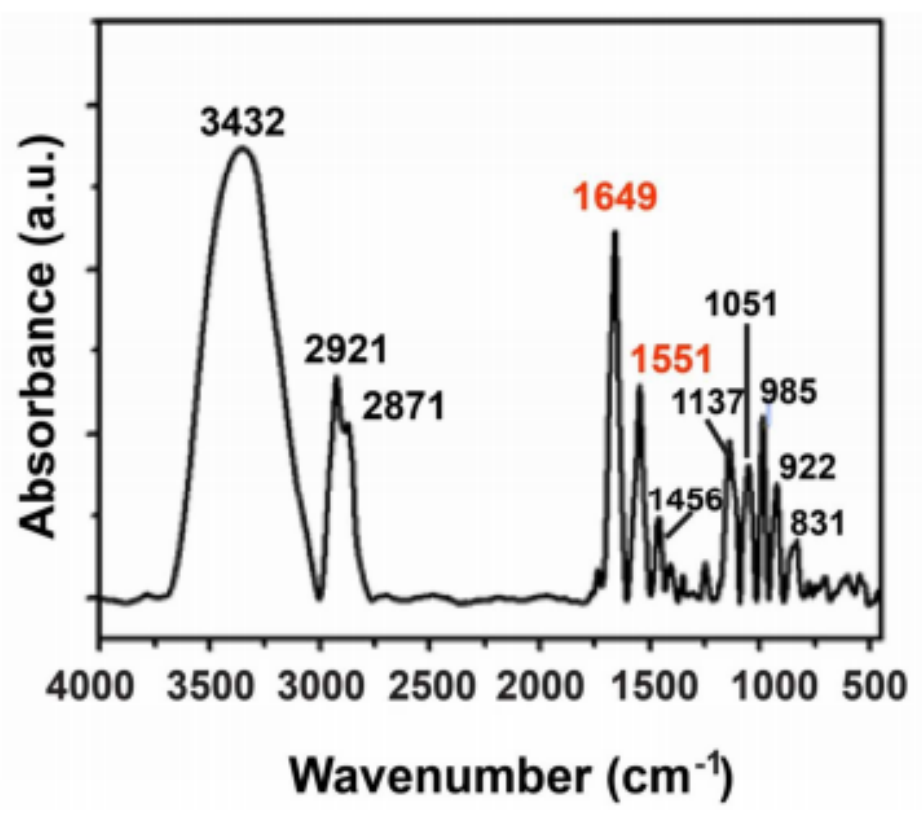

Figure 2.5. FT-IR spectra of the detection antibody functionalized SERS probe. The peaks at 1649 and $1551 \mathrm{~cm}^{-1}$ : correspond to the N-H bending in the antibody.

The coating of the silica layer was further approved by the UV-visible absorption spectra. That is, the localized surface plasmon resonance (LSPR) absorption band of the nanoparticles shifted from 770 to $790 \mathrm{~nm}$ after it was coated with silica (Figure 2.3). Next, $100 \mu \mathrm{L}$ of TEPSA was added to the SERS probe solution and then incubated overnight to achieve the carboxylgroupterminated SERS probes. In the current study, the conjugation of carboxyl-group onto the surface of SERS nanoparticles was confirmed by FT-IR spectra (Figure 2.4). The carboxylgroupterminated sandwich nanoparticles were then activated by adding a PBS buffer solution containing $50 \mathrm{mM}$ NHS and $200 \mathrm{mM}$ EDC and then incubated for $1 \mathrm{~h}$. Subsequently, $100 \mu \mathrm{L}$ of $1.0 \mathrm{mg} / \mathrm{mL}$ detection antibody was added, and the mixture was incubated overnight. After that, the mixture was centrifuged and washed with a PBS buffer three times to remove excessive free antibody. The resulting SERS probe conjugates were stored in $100 \mu \mathrm{L}$ of eluent buffer $(20 \mathrm{nM}$ of $\mathrm{Na} 3 \mathrm{PO} 4 \cdot 12 \mathrm{H} 2 \mathrm{O}$ containing $5 \% \mathrm{BSA}, 0.25 \%$ Tween 20 , and $10 \%$ sucrose) solution for future use. The successful conjugation of detection antibody onto SERS nanoparticles was confirmed by the FT-IR spectra (Figure 2.5). 


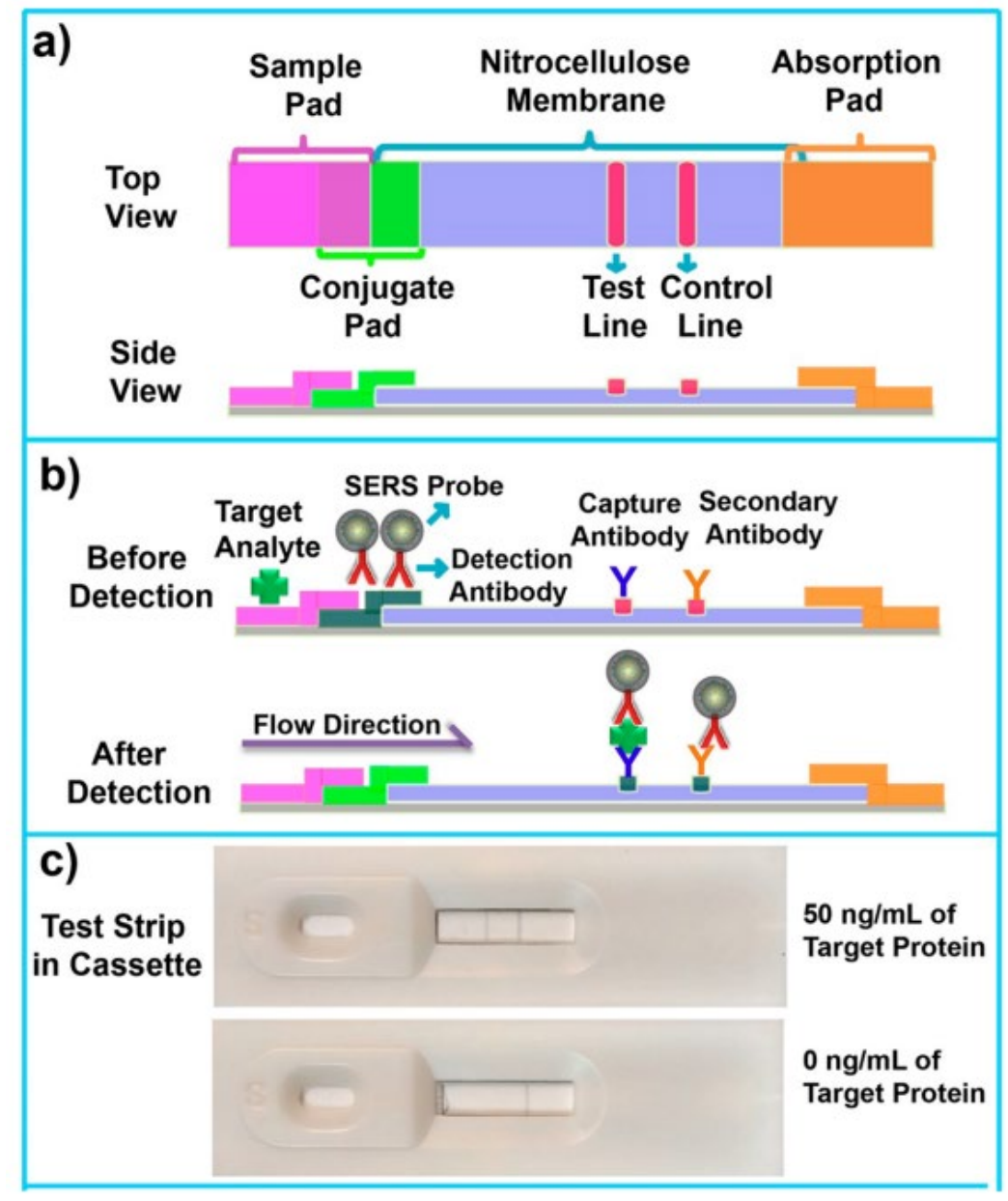

Figure 2.6. Schematic illustration of the operation principle of SERS paper-based lateral flow strip (PLFS). (a) Top and side views; (b) side view before and after detection of protein biomarker; (c) optical photos of PLFS assembled in cassettes in the presence (upper) and absence (bottom) of the target protein.

\subsubsection{Preparation of Test Strip}

Figure 2.6 shows the schematic diagram of the PLFS, which consists of four components: a sample pad, a conjugate pad, a nitrocellulose membrane (NC membrane), and an absorbent (or wick) pad. All the components were mounted on a common backing layer (typically an inert plastic, e.g., polyester). The sample pad was saturated with a Tris-HCl buffer ( $\mathrm{pH} 8.0$ ) containing $0.23 \%$ Triton X-100 and 0.05 and $0.15 \mathrm{M} \mathrm{NaCl}$. The pad was then dried at $37{ }^{\circ} \mathrm{C}$ for $2 \mathrm{~h}$ and stored in a desiccator at room temperature (RT). Then, $200 \mu \mathrm{L}$ of $2.5 \mathrm{mg} / \mathrm{mL}$ capture antibodies and secondary antibodies were dispensed onto the NC membrane by a homemade dispenser to 
form a test line and a control line, respectively. The capture antibody and the secondary antibody were dispensed twice and once, respectively. The distance between the test line and control line was $3 \mathrm{~mm}$. The membrane was then dried at $37^{\circ} \mathrm{C}$ for $1 \mathrm{~h}$ and stored at $4{ }^{\circ} \mathrm{C}$ under a dry condition. Successful conjugation of antibody onto the NC membrane was confirmed by FT-IR spectra (Figure 2.7), in which the peak at $1534 \mathrm{~cm}^{-1}$ indicated the $\mathrm{N}-\mathrm{H}$ bending. The SERS probes conjugated with the detection antibodies were dropped onto the conjugate pad. Finally, all the four processed components were assembled onto a plastic adhesive backing layer. The components were overlapped with $2 \mathrm{~mm}$ to ensure that the solution was able to flow through the whole strip. Each strip was cut to be $3 \mathrm{~mm}$ wide by a paper cutter (Swingline $12 \mathrm{in}$. ClassicCut Lite Trimmer). Before testing, $7 \mu \mathrm{L}$ of SERS probes conjugated with the detection antibody was loaded onto the conjugate pad of PLFS. During testing, $100 \mu \mathrm{L}$ of sample solution containing a certain concentration of analytes was dropped on the sample pad of PLFS.

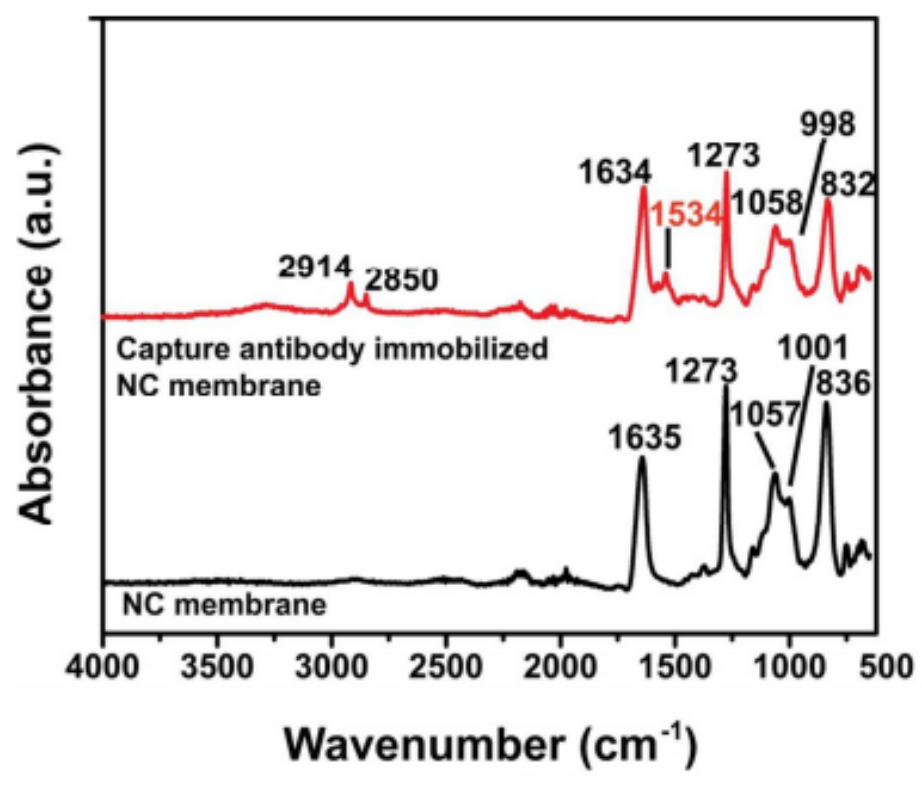

Figure 2.7. FT-IR spectra of the capture antibody functionalized NC membrane. The peak at $1534 \mathrm{~cm}^{-1}$ is assigned to the $\mathrm{N}-\mathrm{H}$ bending.

\subsection{RESULTS AND DISSCUSSION}

\subsubsection{Operation Principle}

The prepared PLFS is a carrier device containing dry reagents that are activated by applying a fluid sample (Figure 2.6b). Once the sample solution containing analyte is dropped onto the 
sample pad of PLFS, the solution will flow to the conjugate pad by the capillary force. On the conjugate pad, the target protein molecules interact with the SERS probes conjugated with detection antibodies to form a complex of target protein-detection antibodies labeled SERS probes. The resulting complex continues to move until it is captured by the capture antibody on the test line. At this moment, the SERS probes are accumulated on the test line through the formation of a sandwich-type configuration of SERS probe (linked with detection antibody)protein-capture antibody. The excessive SERS probes linked with detection antibodies continue to migrate and then are captured by the control line where the secondary antibody is immobilized. As a result, two greyish bands are displayed on the test line and the control line, respectively (Figure 1c). A portable Raman spectrometer is employed to record the SERS signal. Under excitation by a $785 \mathrm{~nm}$ laser, the SERS signal is generated from the MBA (Raman reporter) molecules inside of the SERS probes. As shown in Figure 2.8, the SERS spectra acquired from the test line are characteristic of both the Raman reporter and the NC membrane. The amount of SERS probes captured on the test line is in proportion to the concentration of a target protein, which is employed for quantification. In the absence of the target protein, only the control line shows greyish color, indicating that the test strip functions properly. 


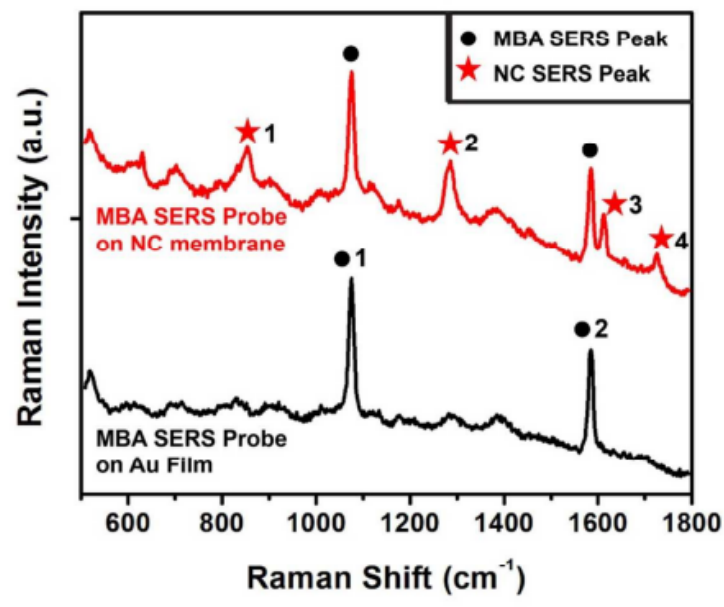

\begin{tabular}{ccc}
\hline & $\begin{array}{c}\text { Peak Position } \\
\left(\mathrm{cm}^{-1}\right)\end{array}$ & Assignment \\
\hline$\star 1$ & 849 & $\mathrm{v}(\mathrm{NO})$ \\
\hline$\star 2$ & 1284 & $\mathrm{v}_{\mathrm{s}}\left(\mathrm{NO}_{2}\right)$ \\
\hline$\star 3$ & 1615 & $\mathrm{v}_{\mathrm{a}}\left(\mathrm{NO}_{2}\right)$ \\
\hline$\star 4$ & 1726 & $\mathrm{v}(\mathrm{CO})$ \\
\hline$\bullet 1$ & 1076 & $\begin{array}{c}\mathrm{v}(\mathrm{CC}) \\
\mathrm{V} 12 \text { Armatic } \\
\text { Ring Vibrations }\end{array}$ \\
\hline$\bullet 2$ & 1585 & $\begin{array}{c}\mathrm{v}(\mathrm{CC}) \\
\mathbf{V}_{\text {sa }} \text { Armatic } \\
\text { Ring Vibrations }\end{array}$ \\
\hline
\end{tabular}

Figure 2.8. SERS spectra of the Au sphere@MBA@silica sandwich SERS probes on the NC membrane and Au film.

\subsubsection{Optimization}

In order to optimize the performance of the PLFS, it was used to detect $1 \mathrm{ng} / \mathrm{mL}$ of human IgG. The human IgG was selected as the model analyte because this protein is well-known, and its testing is much more inexpensive compared with NSE testing. In the optimization study, MIGTC was used as the Raman reporter initially, and the SERS spectra with a characteristic peak at $1173 \mathrm{~cm}^{-1}$ were recorded. The flow rate of the sample solution significantly influences the efficiency of binding between antibodies and antigens, as well as the signal intensity and the signal-to-noise $(\mathrm{S} / \mathrm{N})$ ratio. Generally, the flow rate of a liquid in the PLFS is dependent on the surfactant content and the pore size of the NC membrane. The surfactant in the running buffer is 
an important component, which regulates the binding time between an antibody and its antigen and, meanwhile, mitigates the nonspecific adsorption of SERS probe on the test line. TWEEN 20 was chosen as the surfactant, and its content in the running buffer was optimized. Figure 2.9a reveals that the $\mathrm{S} / \mathrm{N}$ ratio of the SERS peak increased with the growing content of TWEEN 20 content in the running buffer until it reached $0.07 \%$. Further increase in the TWEEN 20 content led to a decrease in the $\mathrm{S} / \mathrm{N}$ ratio, as a high TWEEN 20 content caused a high flow rate of running buffer, leading to a significant decrease in the binding time between the antibody and the antigen. Another parameter governing the flow rate is the pore size of the NC membrane. Reducing the pore size decreases the flow rate and increases the time of binding between the target analyte and the antibody. As shown in Figure 2.9b, the $\mathrm{S} / \mathrm{N}$ ratio for small pores $(0.22 \mu \mathrm{m}$ in a diameter) was higher than that for large pores $(0.45 \mu \mathrm{m})$. In addition, the signal intensity and the detection sensitivity are significantly affected by the detection antibody-linked SERS probe amount on the conjugation pad and the capture antibody immobilized on the test line because both of the factors affect the ability of PLFS to capture the antigen. As shown in Figure 2.9c, d, the optimal detection antibody-linked SERS probe amount was $7 \mu \mathrm{L}$, and two-time dispenses were the best.

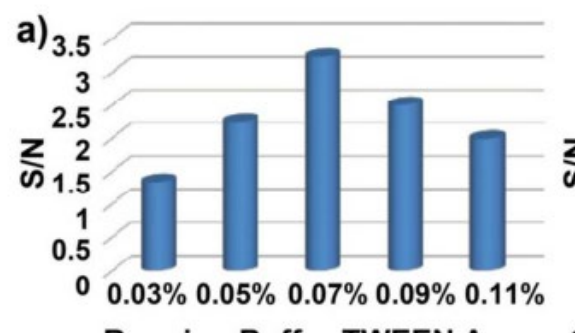

b)

c)
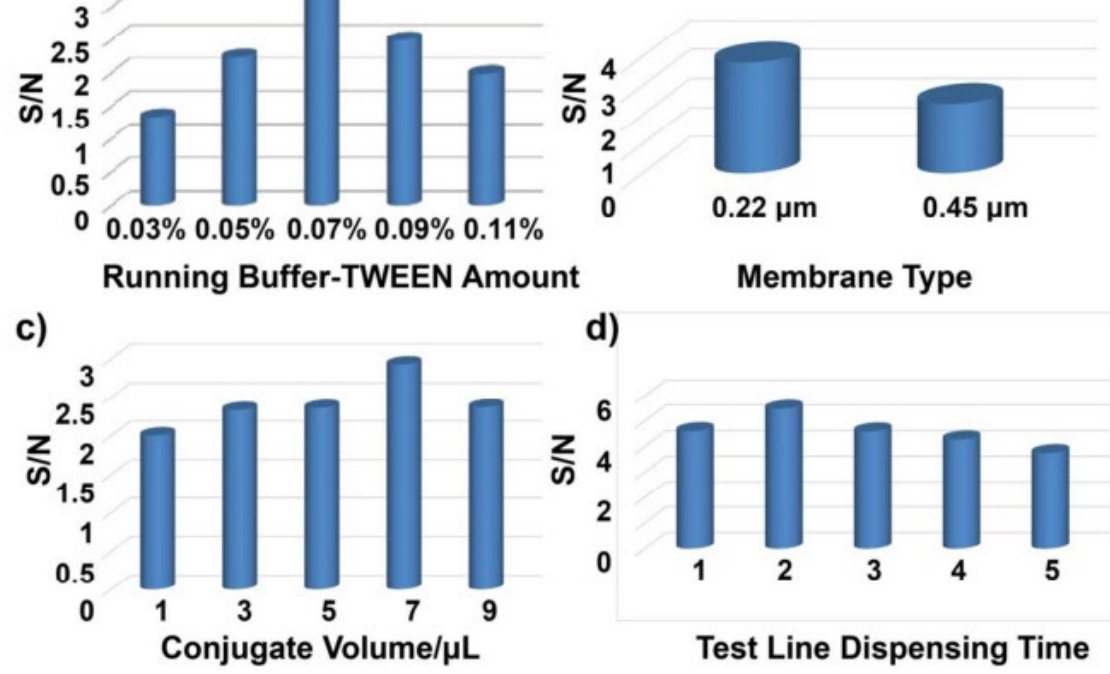

d)

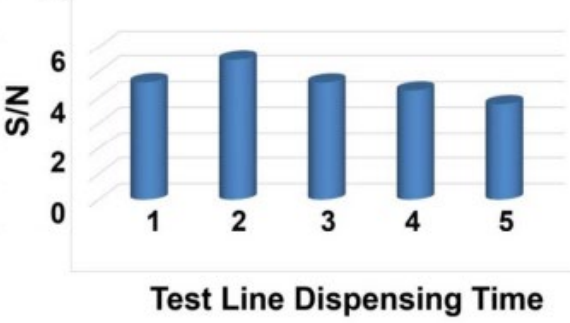

Figure 2.9 Optimization of the experiment conditions for SERS-PLFS. a) Effect of the surfactant TWEEN 20 content in the running buffer on the $\mathrm{S} / \mathrm{N}$ ratio of PLFSs, b) effect of the membrane type (pore size) on the $\mathrm{S} / \mathrm{N}$ ratio, c) effect of the volume of SERS-detection antibody conjugate on the $\mathrm{S} / \mathrm{N}$ ratio, and $\mathrm{d}$ ) effect of the dispensing time of the capture antibody on test line on the 
$\mathrm{S} / \mathrm{N}$ ratio.

\subsubsection{Performance of Test Strip}

A comparative study was performed on the use of two types of Raman reporters (MGITC and MBA). The SERS-PLFS with two different Raman reporters were used to measure various concentrations of human IgG in the PBS buffer. Figure 2.10 shows the MBA-SERS spectra and the corresponding calibration curve (Figure 2.10b). The overall SERS intensity obtained from the MBA-based PLFS (Figure 2.10a) was stronger, and the peaks were much sharper than those from the MGITCbased PLFS (Figure 2.10c). The calibration curve in Figure 2.10b was obtained by plotting the SERS peak intensity at $1076 \mathrm{~cm}^{-1}$ from MBA as a function of the logarithmic concentration of human IgG. The calibration curve in Figure 2.10d was obtained from the PLFS by recording the SERS peak intensity at $1173 \mathrm{~cm}^{-1}$ from MGITC. The slope of calibration curve for the MBA-PLFS was 15 times higher than that for the MGITCPLFS. The limit of detection (LOD) was determined at the signal-to-noise ratio of 3, which was 0.02 and $0.05 \mathrm{ng} / \mathrm{mL}$ for the MBA-PLFS and the MGITC-PLFS, respectively. In short, the MBA-PLFS exhibited higher sensitivity and lower LOD than the MGITC-PLFS. Therefore, MBA was employed for analyte detection instead of MGITC in the following experiments. The reproducibility of the MBA-based PLFS was examined. Human IgG samples at three levels were tested, and five SERS spectra were acquired at each level of human IgG. The SERS peak

intensity of MBA at $1076 \mathrm{~cm}^{-1}$ exhibited good signal reproducibility. The corresponding relative standard deviation (RSD) values of MBA peak intensities were $7.0 \%, 4.1 \%$, and $5.9 \%$ for measuring 10,1 , and $0 \mathrm{ng} / \mathrm{mL}$ of $\mathrm{IgG}$ in PBS, respectively. 

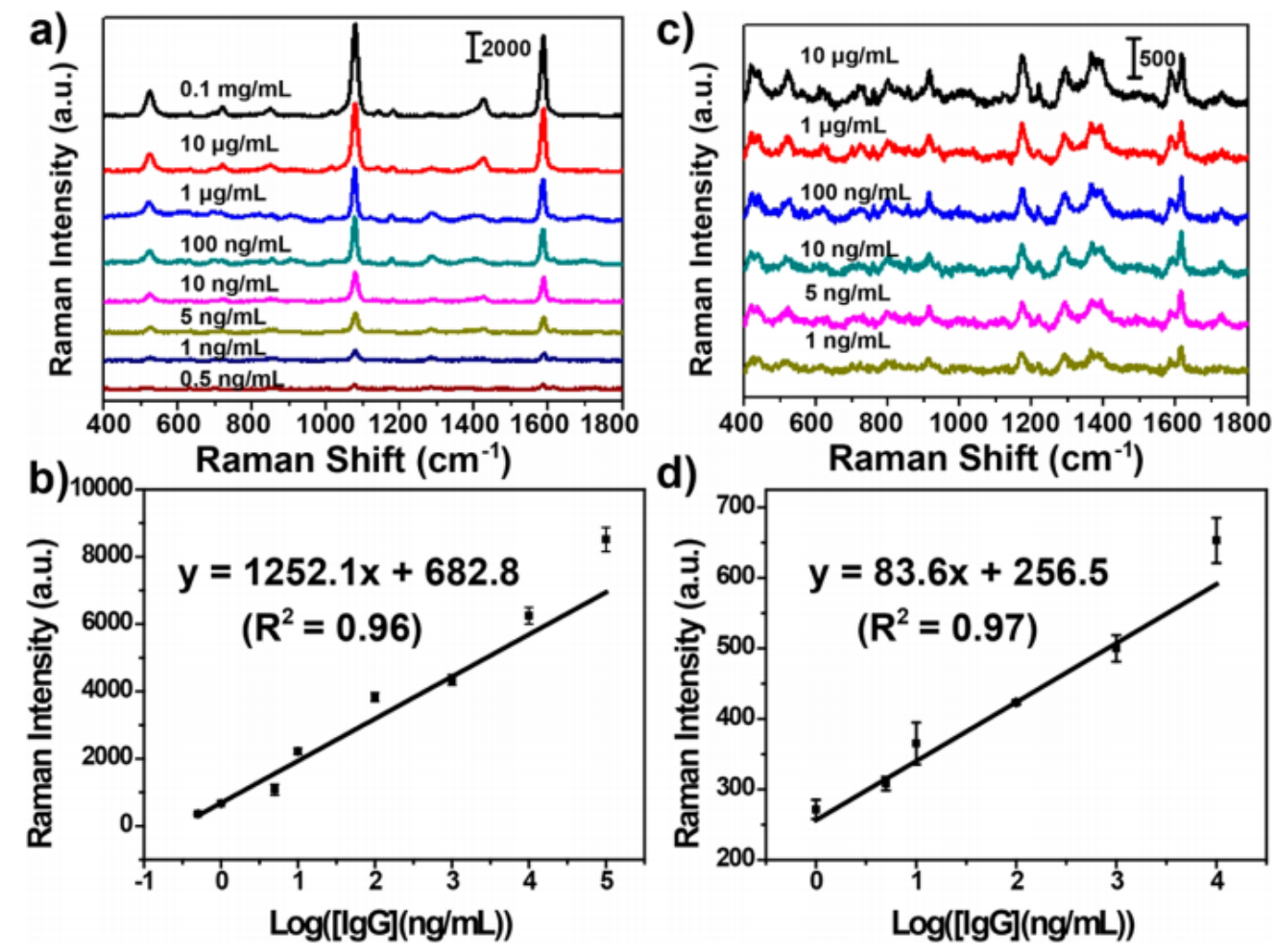

Figure 2.10. SERS spectra obtained from the SERS-PLFS with different Raman report molecules. (a) MBA as Raman reporter and the corresponding calibration curve (b); (c) MGITC as Raman reporter and the corresponding calibration curve $(\mathrm{d})$.

\subsubsection{Measurement of Anti-Interference Ability in Blood Plasma.}

In order to study the anti-interference ability in real-world biological fluids, the developed SERS-PLFS was employed to measure the human IgG spiked in a PBS solution and in a mixture of $90 \%$ PBS and 10\% blood plasma (a specially treated IgG-free blood plasma purchased from US Biological Life Science (P4252-56 Plasma, Human, IgG Free)), respectively. When the SERS-PLFS was used for detection of IgG in 100\% PBS solution, the calibration curved showed a linear detection range from $0.5 \mathrm{ng} / \mathrm{mL}$ to $0.1 \mathrm{mg} / \mathrm{mL}$ (Figure 2.10b), achieving a LOD of 0.02 $\mathrm{ng} / \mathrm{mL}$. When it was used to test a mixture of $90 \%$ PBS and $10 \%$ blood plasma, the calibration curve showed a linear detection range from $1.0 \mathrm{ng} / \mathrm{mL}$ to $0.05 \mathrm{mg} / \mathrm{mL}$ (Figure 2.11), achieving a LOD of $0.08 \mathrm{ng} / \mathrm{mL}$. In addition, the slope of the calibration curve obtained from the mixture of 90\% PBS and 10\% blood plasma (Figure 2.11) was much smaller than that from $100 \%$ PBS (Figure 2.10b). The results indicated that an abundance of biomolecules present in blood plasma 
affected the binding of antibody to the target protein.

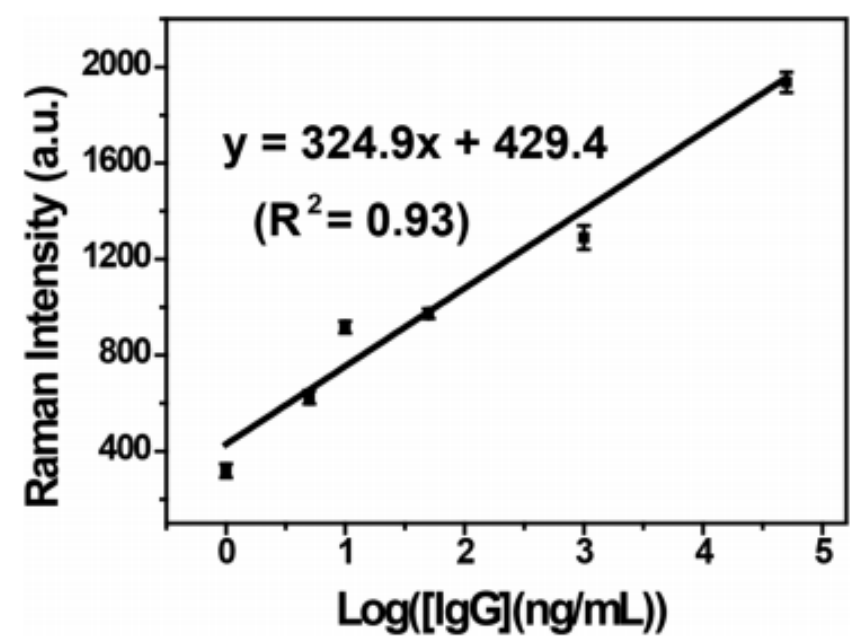

Figure 2.11. Calibration curve of SERS-PLFS with MBA as Raman reporter for detection of IgG in a mixture of $90 \%$ PBS and $10 \%$ blood plasma.

\subsubsection{Comparison of Performance}

Comparative studies were performed on the colorimetric and SERS PLFS. The plasmonic Au nanoparticles acted as the sensing transducer in the colorimetric PLFS while the $\mathrm{Au}$ nanostar@MBA@silica sandwich nanoparticles served as the sensing transducer in the SERSPLFS. Figure 2.12a shows that the colorimetric PLFS worked well when it was used to measure IgG in the PBS solution. The calibration curve showed a linear range from $1 \mathrm{ng} / \mathrm{mL}$ to $50 \mu \mathrm{g} / \mathrm{mL}$ (Figure 2.12c), achieving a LOD of $0.5 \mathrm{ng} / \mathrm{mL}$. However, when the colorimetric PLFS was employed for IgG detection in a mixture of 90\% PBS and 10\% blood plasma, the color intensity of the test line was much weaker than that tested in a PBS buffer (Figure 2.12b), and a high LOD (50 ng/ mL) was observed. When the SERS-PLFS was used for detection of IgG in 100\% PBS solution, a LOD of $0.02 \mathrm{ng} / \mathrm{mL}$ (Figure 2.10b) was achieved, which was 1 order of magnitude lower than that of the LOD $(0.5 \mathrm{ng} / \mathrm{mL}$, Figure 2.12c $)$ of the colorimetric PLFS. In contrast, when they were applied to the sample matrix containing blood plasma, the SERS-PLFS (Figure 2.11) showed much better performance than the colorimetric PLFS (Figure 2.12c). The SERS-PLFS exhibited a LOD of $0.08 \mathrm{ng} / \mathrm{mL}$, which was 3 orders of magnitude lower than that $(50 \mathrm{ng} / \mathrm{mL})$ of the colorimetric counterpart. This result demonstrated that the SERS-PLFS had much better antiinterference ability than the colorimetric counterpart when they were applied to the blood plasma sample matrix. 
a) in buffer

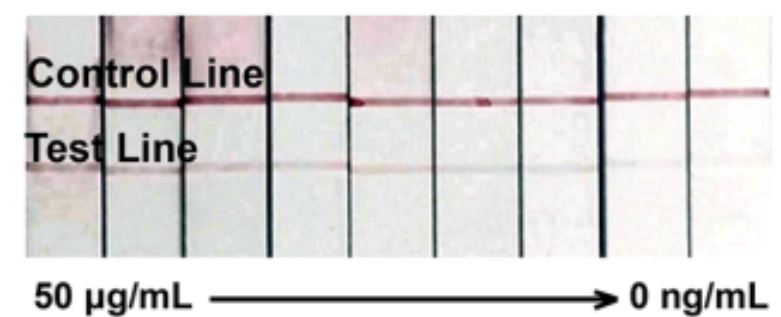

b) in plasma
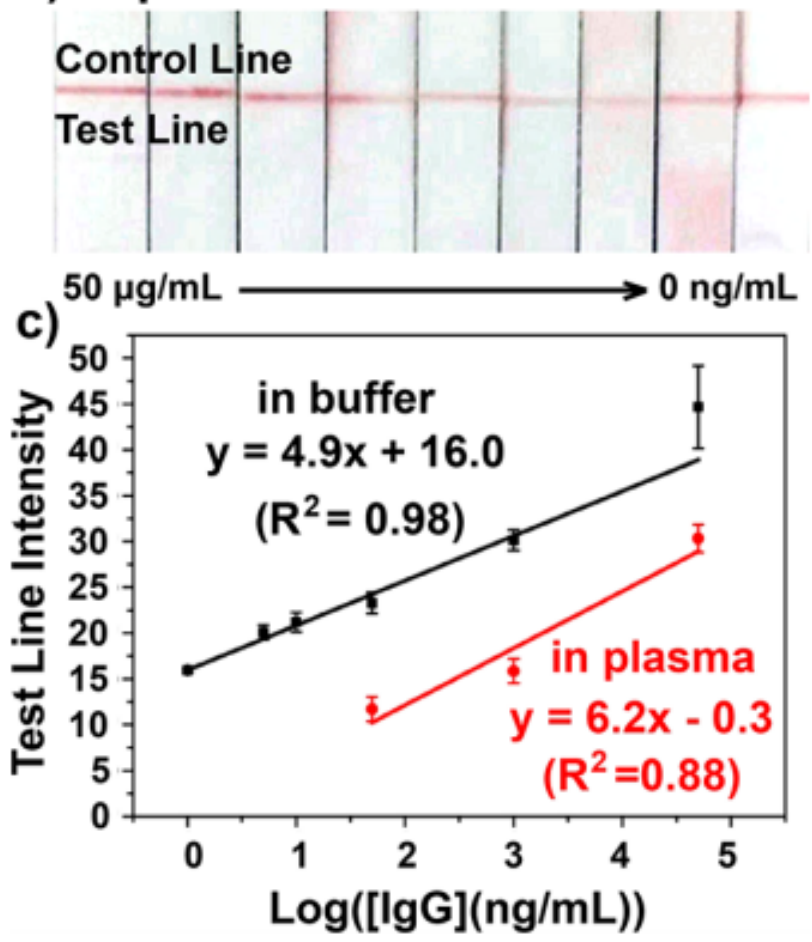

Figure 2.12. Optical photos and calibration curves of colorimetric PLFS for detection of $\operatorname{IgG}$ $(0-50 \mu \mathrm{g} / \mathrm{mL})$ : (a) in PBS buffer and (b) in a mixture of $90 \%$ PBS and $10 \%$ blood plasma; (c) corresponding calibration curves.

\subsubsection{Measurement of Protein Biomarker in Diluted Blood Plasma.}

The SERS probe is the key to the SERS-PLFS. In our sandwich-structured SERS probe, the silica shell is vital to ensure the stability of the SERS probe in the real-world blood plasma that typically exhibits high ionic strength, as shown in our previous paper. Herein, the stability of the bare $\mathrm{Au}$ nanospheres, the silica-coated Au nanospheres, and the silicacoated Au nanostars in the diluted blood plasma was tested (see Figure 2.13). It was found that the color of the $80 \%$ blood plasma sample containing the bare Au nanosphere became darker after $6 \mathrm{~h}$ of incubation, and the 
bare $\mathrm{Au}$ nanospheres were found to be aggregated at the bottom of the vial after overnight. In contrast, the color of the counterpart samples containing the silica-coated Au nanospheres and the silicacoated $\mathrm{Au}$ nanostar was not altered, and no aggregation was observed after overnight. This result indicated that the silica shell prevented the nanoparticles from aggregation in the diluted blood plasma.
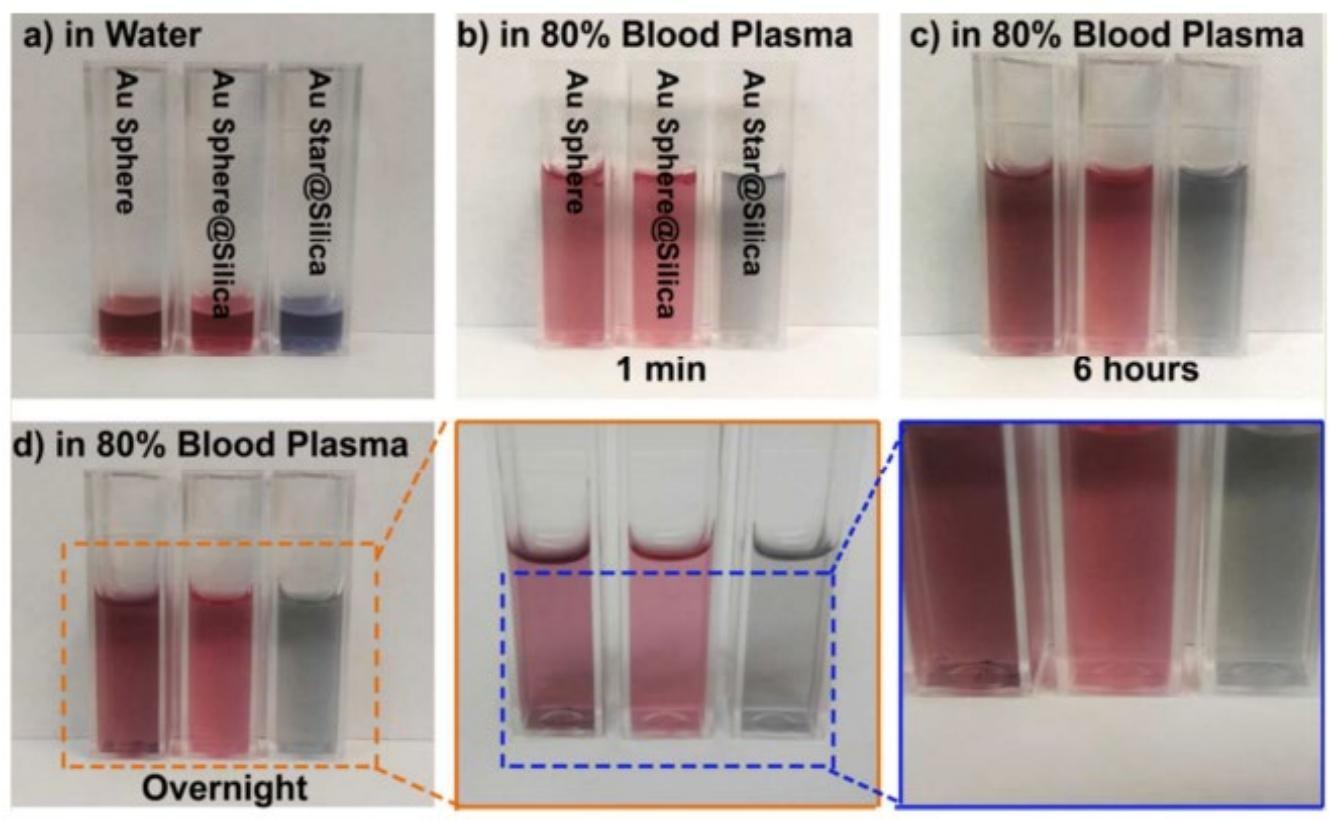

Figure 2.13. Stability test results of the bare Au nanospheres, the silica-coated Au nanospheres and the silica-coated Au nanostars. (a) nanoparticles in water; (b) nanoparticles in a mixture of $80 \%$ of blood plasma and $20 \%$ buffer solution after sitting for 1 minute; (c) nanoparticles in the mixture after 6 hours; and (d) nanoparticles in the mixture after overnight.

In the SERS probe, the $\mathrm{Au}$ nanostar is chosen as the core rather than the $\mathrm{Au}$ nanosphere because the Au nanostar can result in much higher SERS enhancement than the Au nanosphere. ${ }^{42}$ This is due to the fact that the Au nanostar possesses a lot of "hot spots" and shows a LSPR band that is overlapped with the wavelength of excitation laser. Under the optimal experiment condition, the developed SERS-PLFS was employed to detect various concentrations of NSE biomarker spiked into a mixture of $20 \%$ PBS solution and $80 \%$ blood plasma. It is noted that measurement of NSE in a diluted blood plasma can be finished within $30 \mathrm{~min}$. Prior to NSE measurement, the baseline concentration of NSE in the blood plasma was measured by a commercial NSE ELISA kit (R \& D System). Three ELISA measurements were performed. 
None of the tests showed any signal response, which indicated that the NSE content in blood plasma was lower than the LOD $(0.038 \mathrm{ng} / \mathrm{mL})$ of the ELISA Kit. Figure 2.14 shows that the intensity of SERS peak at $1076 \mathrm{~cm}^{-1}$ gradually rises with an increase in the NSE concentration in a mixture of $20 \%$ PBS and $80 \%$ blood plasma. The SERS peak intensity (y) versus the NSE concentration (x) was fitted as $y=6.7 x+275.3$ with the relative coefficient $\left(R^{2}\right)$ of 0.98 , showing a linear detection range from 1.0 to $75.0 \mathrm{ng} / \mathrm{mL}$, achieving a LOD of $0.86 \mathrm{ng} / \mathrm{mL}$. The LOD was estimated with three times the standard deviation of negative control divided by the slope of calibration curve. This LOD was below the cutoff value of NSE concentration (12.4 $\mathrm{ng} / \mathrm{mL}$ ) in blood plasma of TBI patients. The NSE level in TBI patients generally is elevated to $>21.7 \mathrm{ng} / \mathrm{mL}$. Our experimental results show the SERS-PLFS developed in the present work can work for NSE detection in blood plasma.
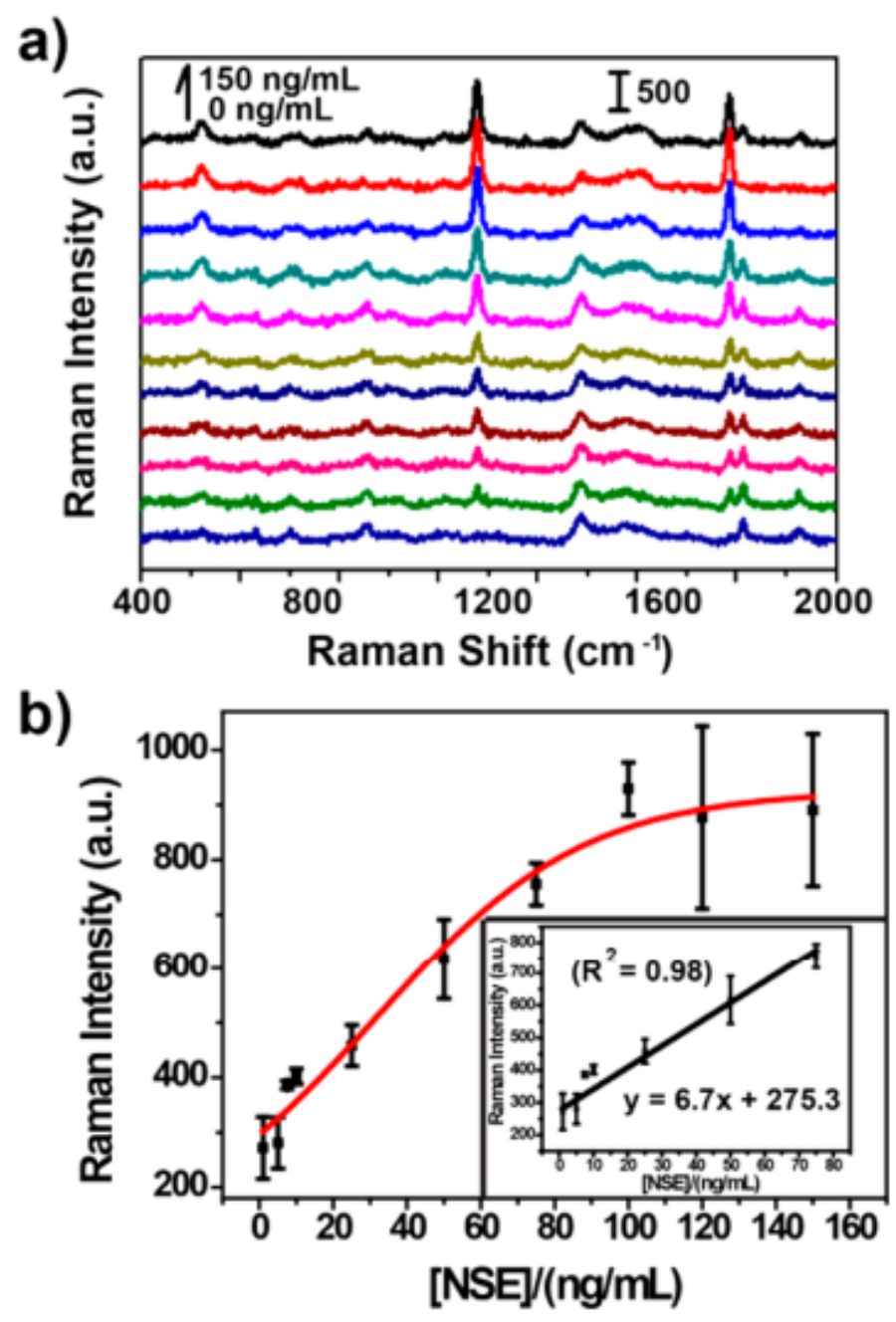

Figure 2.14. SERS spectra (a) and calibration curve (b) of SERS-PLFS with MBA as Raman 
reporter for detection of NSE in a mixture of $20 \%$ PBS and $80 \%$ blood plasma.

\subsubsection{Measurement of Protein Biomarker in Clinical Blood Plasma Samples}

The calibrated SERS-PLFS was employed to measure the NSE biomarker level in clinical blood plasma taken from the TBI patients. The same clinical samples were also measured with the commercial ELISA kits to validate the measurement results obtained from the SERSPLFS. Figure 2.15 shows the NSE concentrations in six clinical blood plasma samples measured by the SERS-PLFS and the NSE ELISA kits (R \& D System and Eagle Bio), respectively. The results reveal that the data obtained by the SERS-PLFS were comparable to those by the ELISA. This result is appealing because the SERS-PLFS has evident advantages over ELISA when applied to clinical blood plasma samples. The SERS-PLFS is easy to operate, which enables clinical blood plasma samples to be measured rapidly with a portable Raman spectrometer. The clinical impact of such an inexpensive and rapid bedside diagnostic tool stands to change practice in the diagnosis of TBI in both the clinical and research arenas through early, easy, and accurate detection of true TBI and will reduce the diagnostic radiation exposure, increase the accuracy of the diagnosis, decrease costs, allow for earlier interventions aimed at mitigating both short- and long-term sequelae, and improve the quality of TBI clinical trials.

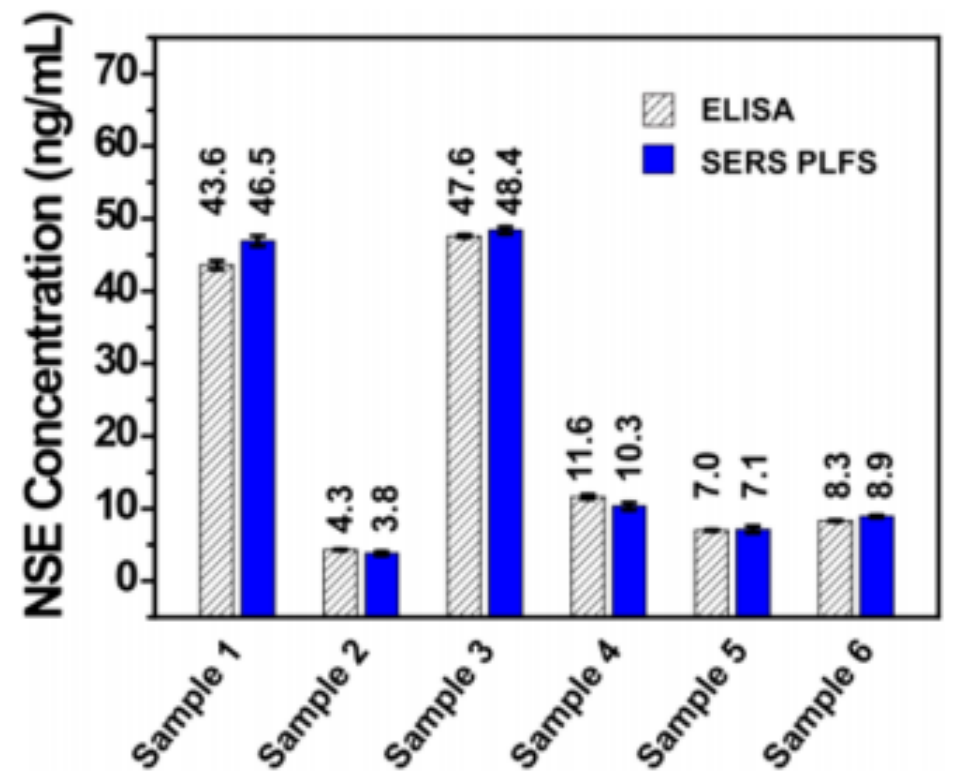

Figure 2.15. NSE concentrations in clinical blood plasma samples, which were measured by ELISA and the SERS-PLFS, respectively. 


\subsection{CONCLUSION}

In summary, a SERS-PLFS was successfully developed in the present work. The LOD of SERS-PLFS was 1 order of magnitude lower than that of the colorimetric PLFS when they were used for detection of proteins in PBS buffer but was 3 orders of magnitude lower in a mixture of PBS and blood plasma. This result proves that the SERS-PLFS had much better anti-interference capability and improved sensitivity in blood plasma-containing sample matrix compared to the colorimetric PLFS. The key to success of SERS-PLFS is the employment of the $\mathrm{Au}$ nanostar@Raman reporter@silica sandwich nanoparticles as the SERS probes. Such SERS probes have strong SERS enhancement capability, excellent stability in complex blood plasma matrix, and good ability of antiinterference. The SERS-PLFS achieved a linear detection range from $1.0 \mathrm{ng} / \mathrm{mL}$ to $75.0 \mathrm{ng} / \mathrm{mL}$ toward NSE in a mixture of $80 \%$ blood plasma and $20 \%$ PBS. The SERS-PLFS was successfully employed for detection of NSE in clinical blood plasma samples. Obviously, the SERS-PLFS has advantages over the ELISA approach, including low cost, portability, short operation time, and simple operation procedure. These merits endow the SERS-PLFS with great potential to be employed as a POC tool for monitoring the protein biomarkers in real-world blood samples. 


\section{Chapter 3: PLASMA SEPARATION UNIT IN WHOLE BLOOD TESTING}

\subsection{BIOMARKER OF CARCINOEMBRYONIC ANTIGEN (CEA)}

A critical and challenging future direction in science and engineering is to develop technologies to improve the health of people in the developing and remote regions of the world. ${ }^{1}$ However, advanced diagnostic technologies are always centralized and requiring sophisticated instruments, specialized laboratory personnel and expensive chemicals. Moreover, continuous and long term monitoring add tremendous time and financial burden on patients due to soaring costs and frequent travel to hospitals and institutions. These factors have accelerated the emphasis on the development of low-cost, easy-to-operated and reliable devices to shift diagnostic testing from medical institutes and laboratories to the sites of patient care.

Paper-based devices appear to be effective tools for providing access to point-of-care (POC) applications, owing to their advantages of low cost, fast response, portability and ease of use. ${ }^{43}$ In addition, paper is compatible with biological samples, which allows its application in analyzing various biomolecules during bioassay. ${ }^{44}$ Moreover, paper-based POC testing allows test results to be read out facilely and quickly, which assists in making clinical management decisions in an immediate and timely manner. The underlying mechanism of a paper-based POC device is mainly based on sensing of a specific target molecule or biomarker from human fluids, such as blood, serum, plasma, saliva and urine, which are easy to be collected and provides diverse sensing pathways in a less-invasive mode. Nevertheless, issues arise from poor sensitivity and the interference biomolecules from the complex human fluids, especially red blood cells (RBCs) from whole blood samples. For example, paper substrates are easy to be visibly contaminated with the strong red color from RBCs, which leads to imprecise testing results, particularly when the tests are carried out in colorimetric based assays. Additionally, the aggregation of erythrocytes in the optical detection zone can substantially decrease signal quality. In order to wash away the aggregates on the test area, extra washing steps are required, which increases sample-to-answer times. Moreover, most of the clinical biochemical tests are normally operated in the liquid portion of blood without cells, such as blood serum or plasma. Thus, separating RBCs from whole blood is necessary and it is also crucial to elevating the performances of sensors. Conventional separation strategies, such as mechanical forces, electroosmotic flow, dielectrophoretic techniques and magnetic interactions, can only be carried out in 
controlled central laboratories or care centers, where are equipped with sophisticated instruments and specialized laboratory personnel. ${ }^{45}$ Thus, significant effort has been devoted into the development of low-cost and easy-to-operated approaches, which has resulted in the rapid development of miniaturized devices. Recently, several research groups have developed aggregation based approaches, which allows the interception of RBCs, meanwhile enables the separation of plasma from whole human blood samples on paper devices. ${ }^{46}$ The principle of this method is to develop RBC aggregations, which size is too large to pass through the pores within the fiber network of paper. For instance, a specific interaction between antibody and antigen was utilized to trigger RBC haemagglutination inside of the fiber network of paper. However, this method is only capable of separating RBCs from blood samples of types A, B, AB and O Rh positive, which requires blood typing before operations. In addition, antibody immobilized papers require critical fabrication and storage conditions, which increases costs of such devices. Another method was developed based on introducing salt solutions to achieve the goal of aggregating RBCs from whole blood samples. The basic mechanism is based on destabilizing RBC suspension through suppressing the electric double layer on the surface of RBCs; meanwhile generating of osmotic pressure, which causes RBCs to change morphologies, leading to close packing and formation of RBC aggregations. As a result, plasma moves forward by itself in the fiber network through capillary force. ${ }^{47}$ However, this method is difficult to be employed into nanoparticle functionalized paper devices, since the high ionic strength from salt solutions also induces the aggregation of nanoparticles, which leads to inaccurate signal responses and decreased sensitivity. Recently, our research group developed Au nanostar@Raman Reporter@silica sandwich nanoparticles, in which Raman molecules are sandwiched between an Au nanostar and a thin silica shell. ${ }^{43,48}$ The silica coating can stabilize nanoparticles in aqueous solutions with high ionic strength and complex human fluids, leading to the improved stability of the device.

In the current study, we developed a blood plasma separation unit (PSU) integrated paper based lateral flow strip (PLFS) for carcinoembryonic antigen (CEA) detection from whole human blood, which is an important tumor associated protein biomarker. ${ }^{49}$ In general, CEA circulates with elevated concentrations in the fluids of patients with malignancies, such as colon and rectal, ovary, breast, lung cancer and pancreas cancers. ${ }^{50}$ High apparent separation efficiency and separation yield of the PSU were achieved through the employment of three layers of blood 
filtration membranes, which are designed specifically to separate plasma from whole blood samples. The membranes in the PSU were respectively functionalized with salt (sodium chloride), surfactant (TWEEN 20) and salt (sodium chloride). The first layer of membrane with salt functionalization was utilized to trapping RBCs in its fiber network; while the second layer with surfactant was used to accelerate the separated plasma to pass through the second layer. The third layer was also immobilized with salt, which was employed to ensure that the escaped RBCs can be trapped inside of the PSU. The three-layer construction guarantees that the PSU is capable of separating blood plasma with high efficiency and separation rate. During CEA detection, SERS nanoparticles (Au nanostar@Raman Reporter@silica sandwich nanoparticles) were utilized as signal reporters, which endows high sensitivity and strong anti-interference ability to the PSU-PLFS in whole blood samples. As a result, a LOD of $1 \mathrm{ng} / \mathrm{mL}$ of CEA was obtained, which was below the cut-off value that presents in the blood samples of cancer patients. Moreover, owing to the advantages of PLFS, the PSU-PLFS was capable of sensing CEA directly from human whole blood without any sample-pretreatment, which shows a great potential in the POC setting.

\subsection{REAGENT AND INSTRUMENTS}

N-hydroxysuccinimide (NHS), 1-ethyl-3-(3-dimethylaminopropyl) carbodiimide (EDC) and tetraethyl orthosilicate (TEOS) were purchased from Sigma-Aldrich; $\mathrm{HAuCl}_{4}, \mathrm{Na}_{3} \mathrm{PO}_{4} \cdot 12 \mathrm{H}_{2} \mathrm{O}$, Tween 20, Triton X-100, trisodium citrate, PBS $(\mathrm{pH}=7.4)$, Bovine serum albumin (BSA), sodium chloride, streptavidin were purchased from Sigma-Aldrich and used without further purification. Sucrose was purchased from VWR. 4-Mercaptobenzoic acid (technical grade, 90\%) (4-MBA) was purchased from Sigma-Aldrich. 3-Triethoxylsilylpropyl succinic anhydride (TEPSA) was obtained from Gelest Inc.

Carcinoembryonic antigen and anti-CEA antibody were procured from Sigma-Aldrich. Glass fibers (GFCP000800), cellulose fiber sample pads (CFSP001700), laminated cards with Hi Flow plus cards (HF090MC100 with RK12312 Membrane $(0.45 \mu \mathrm{m})$ ), and nitrocellulose membrane (RK13464 $(0.22 \mu \mathrm{m}))$ were purchased from Millipore (Billerica, MA). Whole human blood was purchased from Innovation Inc. 


\subsection{PREPARATION PROCEDURES}

\subsubsection{Preparation of Nanoparticles}
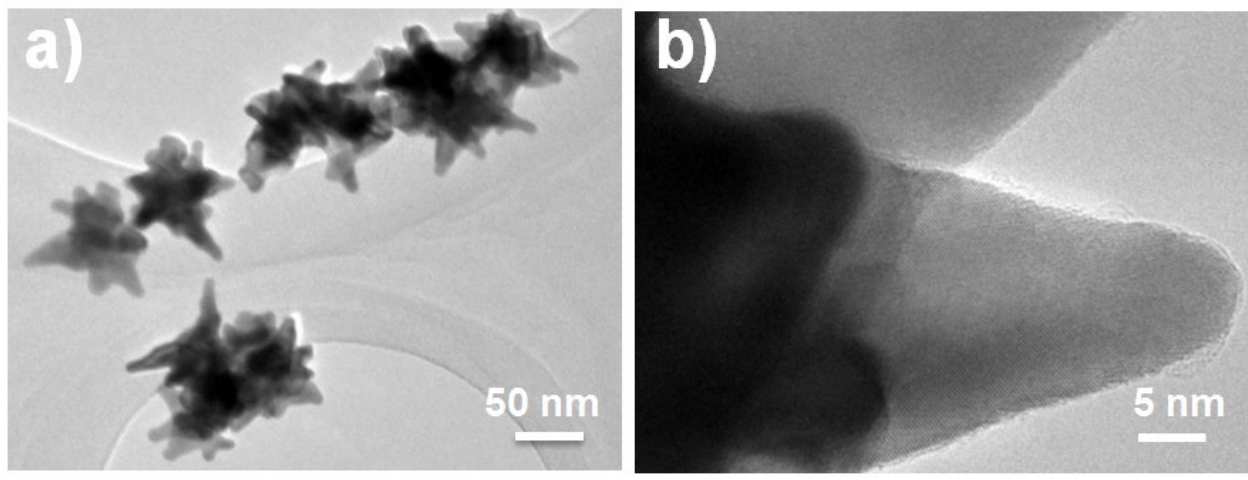

Figure 3.1. TEM images of the Au nanostar@MBA@ $\mathrm{SiO}_{2}$ sandwich nanoparticles a) low magnification and b) high magnification.

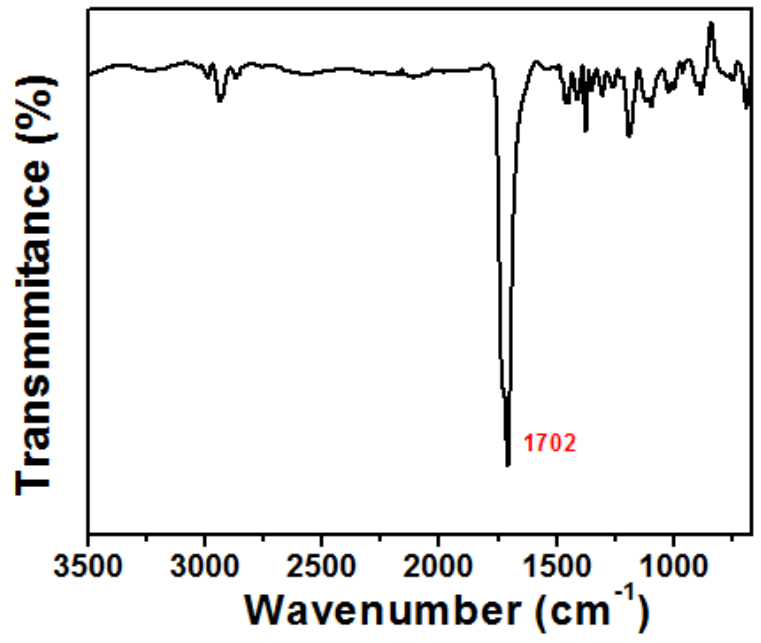

Figure 3.2. FT-IR spectrum of the carboxyl-group labeled SERS NPs. The peak at $1702 \mathrm{~cm}^{-1}$ is assigned to the $\mathrm{C}=\mathrm{O}$ stretching vibration in $\mathrm{COOH}$ of TEPSA.

The Au nanostars, the SERS probes and the detection antibody labelled SERS probes were synthesized according to our previous paper. ${ }^{43}$ The preparation procedure is illustrated in Figure 3.3. Briefly, the sandwich typed SERS probes were synthesized by adding $1.0 \mu \mathrm{M}$ of 4-MBA into the Au nanostar suspension. Then $200 \mu \mathrm{L}$ of $1 \%$ TEOS and $15 \mu \mathrm{L}$ of ammonium solution were added into the mixture with 20 min intervals. After the SERS probes were formed, the resulting solutions were centrifuged and resolved in $500 \mu \mathrm{L}$ of ethanoic solution. $100 \mu \mathrm{L}$ of TEPSA was added to the SERS probe solution and then incubated overnight to achieve the 
carboxyl-groupterminated SERS probes. The successful conjugation of carboxyl-group onto the surface of SERS NPs was confirmed by FT-IR spectra (Figure 3.1). The carboxylgroupterminated sandwich nanoparticles were then activated by adding a PBS buffer solution containing $50 \mathrm{mM}$ NHS and $200 \mathrm{mM}$ EDC and then incubated for $1 \mathrm{~h}$. Subsequently, $100 \mu \mathrm{L}$ of $1.0 \mathrm{mg} / \mathrm{mL}$ detection antibody was added, and the mixture was incubated overnight. After that, the mixture was centrifuged and washed with a PBS buffer three times to remove excessive free antibody. The resulting SERS probe conjugates were stored in $100 \mu \mathrm{L}$ of eluent buffer $(20 \mathrm{nM}$ of $\mathrm{Na}_{3} \mathrm{PO}_{4} \cdot 12 \mathrm{H}_{2} \mathrm{O}$ containing $5 \%$ BSA, $0.25 \%$ Tween 20 , and $10 \%$ sucrose) solution for future use. The morphology and corresponding characterization can be found in our previous published papers. $^{43}$ The successful conjugation of the detection antibody onto the SERS NPs was confirmed by the FT-IR spectrum (Figure 3.2).

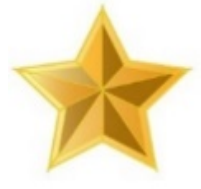

Au Nanostar

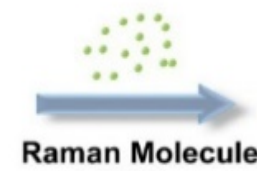
4-MBA

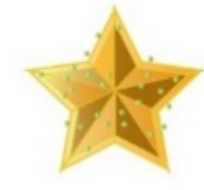

4-MBA Adsorbed Au Nanostar

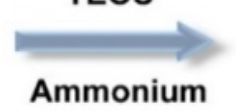

Ammonium

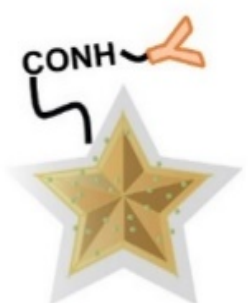

Detection Antibody Linked SERS Probe

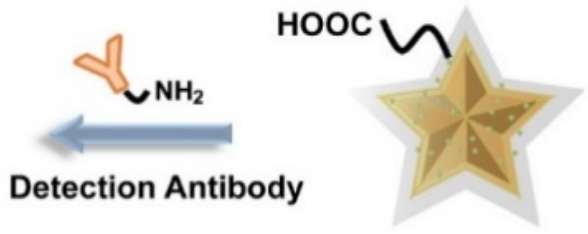

Carboxyl Linked SERS Probe

Figure 3.3. Schematic illustration for synthesis of detection antibody functionalized SERS probes.

\subsubsection{Functionalization of Blood Plasma Filtration Membranes}

A PSU contains three layers of blood filtration membranes (GR membrane). Each membrane was cut with a dimension of $5.5 \mathrm{~mm} \times 3 \mathrm{~mm}$. Two batches of membranes were respectively soaked and incubated in salt solution ( $0.154 \mathrm{M}$ of sodium chloride) and surfactant solution (TWEEN $20(20 \%)$ ) for $1 \mathrm{~h}$. Then the treated membranes were dried at room temperature for $2 \mathrm{~h}$. The resulting membranes were stored in desiccators at room temperature for future use. During 
PSU assembling, each layer of membrane has an overlap of $1.5 \mathrm{~mm}$ with each other (as shown in Figure 3.4).

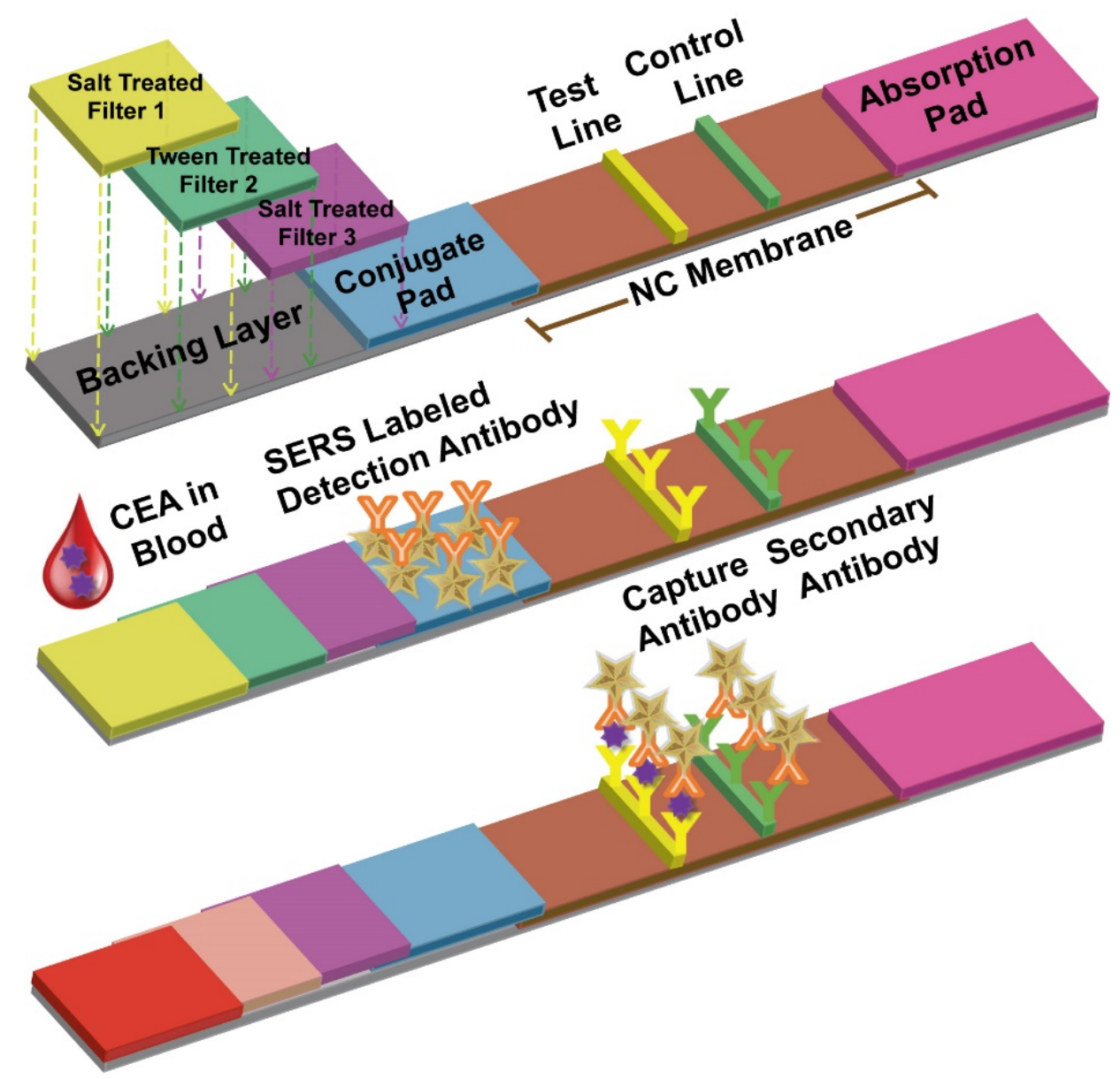

Figure 3.4. Schematic illustration of a PSU-PLFS.

\subsubsection{Preparation of Blood Plasma Separation Unit Modified Test Strip}

A PSU-PLFS contains PSU, conjugate pad, nitrocellulose (NC) membrane and absorption pad. The PSU contains three layers of blood filtration membranes, which were treated with salt, surfactant and salt respectively. Each layer of membrane was laminated on a plastic backing with an overlap of $1.5 \mathrm{~mm}$. As shown in Figure 3.4, the top layer of membrane is modified with salt solution, while the second layer is modified with TWEEN 20. The third layer of membrane is also modified with salt solution, and it has an overlap of $2 \mathrm{~mm}$ with conjugate pad, which ensures that the solution could migrated through PSU to the PLFS. The optical image of the side view of a PSU is illustrated in Figure 3.5. Then the conjugate pad, NC membrane and absorption pad were assembled following the general fabrication procedure of a PLFS. In brief, the test line 
and control line on the NC membrane (Hi Flow plus cards HF090MC100 with RK12312 Membrane $(0.45 \mu \mathrm{m}))$ were prepared by dispensing the solutions of capture antibody and secondary antibody respectively with a home-made dispenser. The distance between the test and control lines was $3 \mathrm{~mm}$. The membrane was then dried at $37^{\circ} \mathrm{C}$ for $1 \mathrm{~h}$ and stored at $4{ }^{\circ} \mathrm{C}$ in a dry state. Finally, the PSU, conjugate pad, NC membrane, and absorption pad were assembled on a plastic adhesive backing $(60 \mathrm{~mm} \times 30 \mathrm{~cm})($ HF000MC100). Each part overlapped $2 \mathrm{~mm}$ to ensure that the solution could migrate through the entire test strip during the assay. Test strips with a 3-mm width were cut using a paper cutter. After a PSU-PLFS was assembled, the solution of detection antibody labelled SERS probes were loaded on the conjugate pad prior to the test.

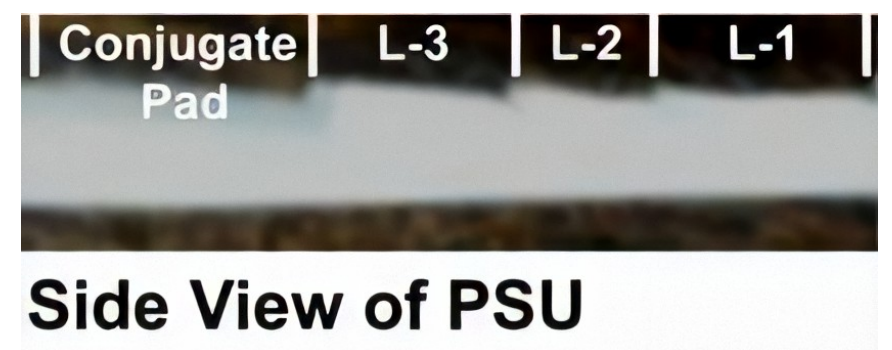

Figure 3.5. Optical image of the side view of a PSU, which contains membrane layer-1, layer-2, layer-3 and conjugate pad.

\subsection{PERFORMANCES}

\subsubsection{Operation Principle}

A PSU-PLFS was comprised of a blood plasma separation unit and a conventional lateral flow test strip. The PSU was utilized to intercept RBCs inside of the unit, while the PLFS was in charge of detecting biomarkers from the separated plasma from the PSU. As shown in Figure 3.4, the PSU contains three layers of blood plasma filtration membranes, which are placed successively in the front edge of a PLFS. During a typical assay, $30 \mu \mathrm{L}$ of blood sample solution containing different concentrations of target antigen was loaded at the first filter layer of the PSU, which was modified with salt. The salt immobilization induced the aggregation of RBCs inside of the fiber network of the membrane; while the separated plasma containing target biomolecules kept migrating along the PSU and then reached the second filter layer. The second layer of filter membrane was immobilized with TWEEN 20, which expedited the separated solution moving forward to the third filter layer. The third filter layer of PSU was also 
functionalized with salt, which guaranteed that the escaped RBCs from the first two filter layers can still be captured inside of the PSU, realizing efficient separation of plasma from whole blood samples. After that, $100 \mu \mathrm{L}$ of buffer was added onto the second layer of filter. Owing to capillary force, the separated plasma containing target antigen with running buffer migrated from PSU to the conjugate pad of the PLFS, where the reaction between target antigens with antibody linked SERS probes occurred. Then the resulted complexes kept moving along with the test strip until they reached the test line and captured by capture antibody through the formation of a sandwich structure (capture antibody-antigen-detection antibody/SERS). The rest of SERS/detection antibody conjugates migrated over the test line and captured by the control line where the secondary antibody was immobilized. During data acquiring, the test strips were analyzed by a portable i-Raman reader. The Raman peak intensity was in proportion to the concentration of target antigen, which was used for quantitative work.

\subsubsection{Apparent Separation Efficiency and Yield}

Separation efficiency and separation yield are the two most critical factors in judging the performances of a blood plasma separation device. In the current study, we utilized the concepts of apparent separation efficiency (ASE) and apparent separation yield (ASY) to evaluate PSU performances for separating RBCs from human blood sample. The processing steps of the ASE of a PSU was illustrated in Figure 3.6. In Step 1, $30 \mu \mathrm{L}$ of human blood sample was firstly loaded on the first layer of filtration membrane on a PSU. After the blood plasma separation was accomplished, a scanner was used to capture the optical images of PSUs. In Step 2, the captured images were converted to grayscale models through the Photoshop software. In Step 3, the images from Step 2 were inverted from black-white model to white-black model. During analysis, the grayscale intensity per unit area of the images in Step 3 were measured and processed using the ImageJ software. Experimental factors, including layers of the blood filtration membranes and the chemical functionalization of the membranes were optimized according to the ASE of a PSU. Since a PSU contains three layers of membrane, therefore, we utilized $\mathrm{ASE}_{21}$ and $\mathrm{ASE}_{31}$ to respectively demonstrate the ASEs between layer 1 and layer 2, and the ASE between layer 1 and layer 3. The $\mathrm{ASE}_{21}$ and $\mathrm{ASE}_{31}$ were calculated according to Equation (1) and (2) below:

$$
\begin{aligned}
& \mathrm{ASE}_{21}=\left(\mathrm{I}_{\text {layer1 }}-\mathrm{I}_{\text {layer2 }}\right) / \mathrm{I}_{\text {layer1 }} \\
& \mathrm{ASE}_{31}=\left(\mathrm{I}_{\text {layer1 }}-\mathrm{I}_{\text {layer3 }}\right) / \mathrm{I}_{\text {layer1 }}
\end{aligned}
$$




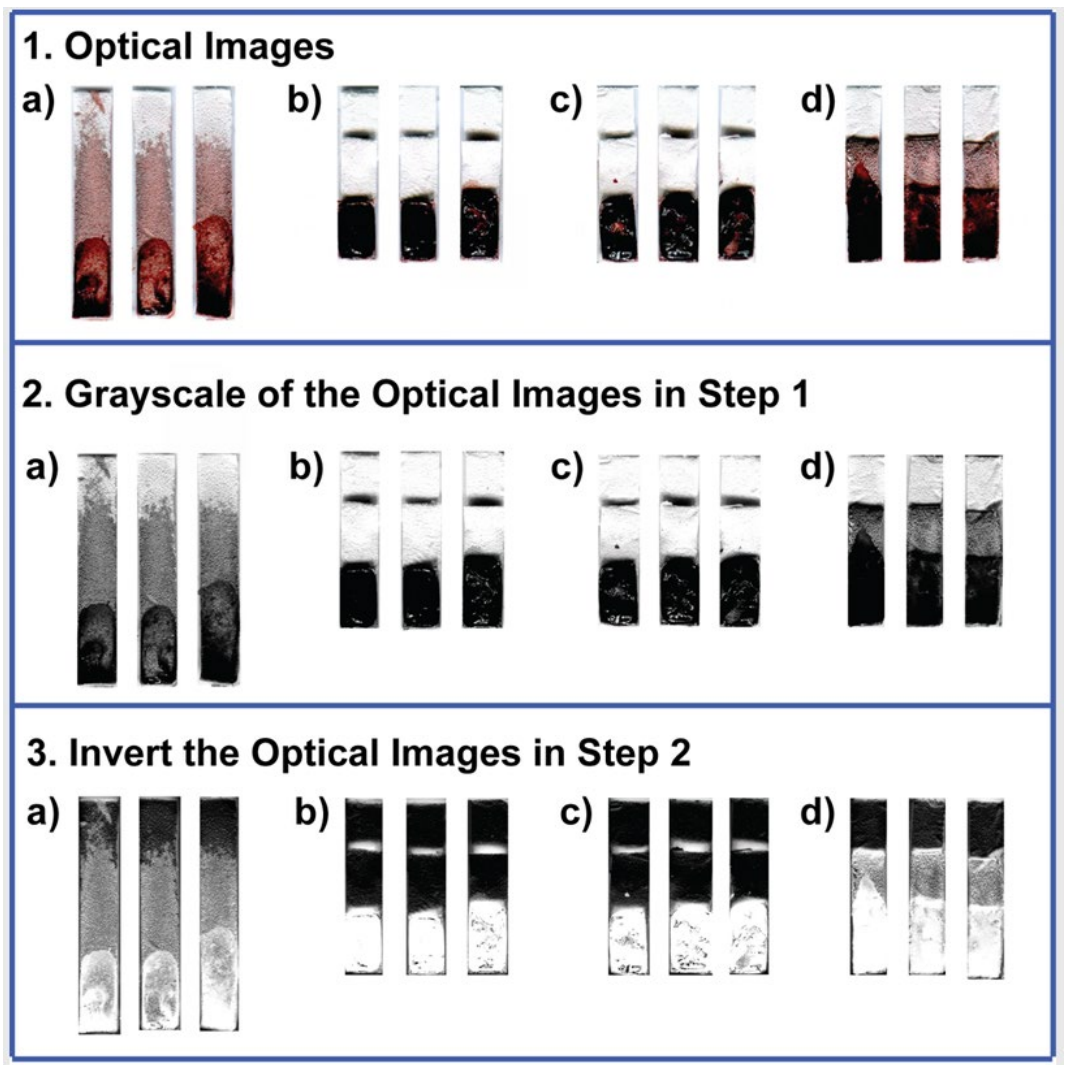

a) 1 layer of filter: treated with salt $(0.154 \mathrm{M})$

b) 3 layers of filters: treated with salt + TWEEN $20+$ salt

c) 3 layers of filters: treated with salt + salt + salt

d) 3 layers of filters: treated with TWEEN $20+$ TWEEN $20+$ TWEEN 20

Figure 3.6. Optimizations and processing steps of ASE of a PSU when human blood sample was loaded. Each blood plasma separation test was repeated three times under the same condition.

Where I represents the intensity of the grayscale in Steps 3 divided by the area of the blood filtration membrane. The PSU was fabricated on the substrates of blood filtration membranes. This kind of membrane was designed specifically for blood filtration through capturing RBCs in the larger pores, while the plasma flows down into the smaller pores on the downstream side of the membrane. Therefore, the optimizations of the filter membranes are crucial for improving the ASE and ASY. Under the same experiment conditions, compared with one layer (Figure 3.7a), three layers of salt treated membranes (Figure 3.7c) exhibited dramatically enhanced $\mathrm{ASE}_{21}$ from $16.1 \%$ to $91.6 \%$, which proved that multiple layers are crucial for elevating the PSU performances. Same conclusion can be drawn when the optical images of PSUs in Figure 3.6a and Figure 3.6c were analyzed. Most of the RBCs can be captured and retained inside of the first membrane layer of the three-layer membrane PSU. On the contrary, only small amount of RBCs 
can be retained at the front edge of the one-layer membrane PSU. The rest RBCs kept migrating along on the membrane surface, which significantly contaminated the unit, leading to poor ASE. The membrane layers from 2 to 5 were also studied through analyzing $\mathrm{ASE}_{12}$ of the PSUs. It can be clearly seen that the $\mathrm{ASE}_{12}$ increases as the layer of membrane increases (Figure 3.8a). Considering the $\mathrm{ASE}_{12}$ performances and cost, 3 layers of filtration membranes was chosen as the optimal layer amount.

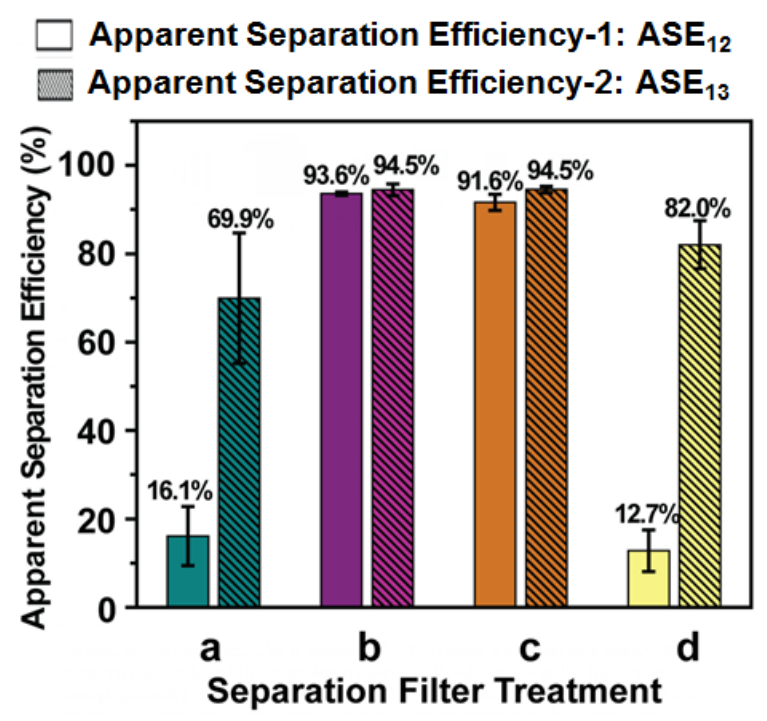

Figure 3.7. $\mathrm{ASE}_{12}$ and $\mathrm{ASE}_{23}$ of a $\mathrm{PSU}$ with different functionalization when human blood sample was loaded. 

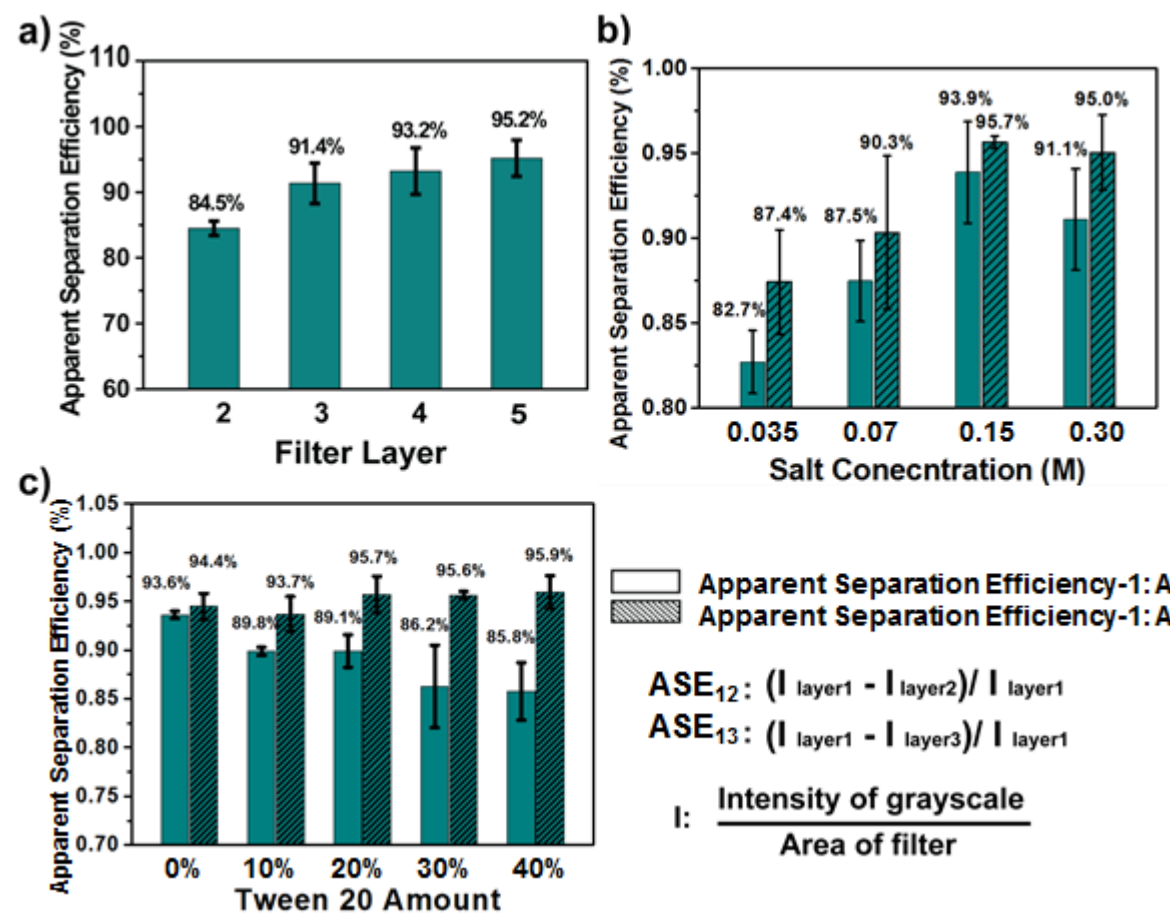

Salt Conecntration (M)

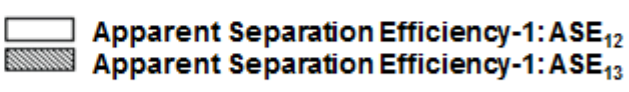

$$
\begin{aligned}
& \mathrm{ASE}_{12}:\left(I_{\text {layer1 }}-\mathrm{I}_{\text {layer2 }}\right) / I_{\text {layer1 }} \\
& \mathrm{ASE}_{13}:\left(\mathrm{I}_{\text {layer1 }}-\mathrm{I}_{\text {layer3 }}\right) / \mathrm{I}_{\text {layer1 }} \\
& \mathrm{I}: \frac{\text { Intensity of grayscale }}{\text { Area of filter }}
\end{aligned}
$$

Figure 3.8. Experimental optimizations of the PSU. The effect of a) different layers of blood filtration membranes; b) $\mathrm{NaCl}$ concentrations; c) TWEEN 20 amount on $\mathrm{ASE}_{12}$ and $\mathrm{ASE}_{13}$.

Chemical treatment is another important factor to improve ASE, especially salt treatment. This is because that RBCs contain a weak negative charge, which contributes to stabilizing RBC suspension in plasma. While salts can destabilize RBC suspension through generating osmotic pressure across the RBC membrane. In addition, salt addition can suppress the electric double layer on the RBC surfaces, which results in the shape change and close packing of RBCs, leading to the aggregation of RBCs. Blood filtration membranes were optimized through employing different concentrations of $\mathrm{NaCl}$ solutions to achieve high ASE. As descripted in Figure 3.8b, with the increase of $\mathrm{NaCl}$ concentration, the $\mathrm{ASE}_{12}$ and $\mathrm{ASE}_{13}$ increase respectively. This is because that with the increase of salt concentration, the degree of aggregation increases, which contributes to elevated ASE. From the figure we can observe that $0.15 \mathrm{M}$ of $\mathrm{NaCl}$ is the optimal salt concentration, which maintained the ASE above 90\%. However, owing to the formation of the $\mathrm{RBC}$ aggregation in the present of salt, the generated $\mathrm{RBC}$ lumps might block the membrane and slow down the rate of plasma separation. Based on this reason, surfactant was introduced to functionalize the second layer of filter membrane for accelerating the separation progress. From the study we found that with the increase of TWEEN 20 amount, the separation rate improved 
accordingly. Although the ASE decreased to some extent, the $\mathrm{ASE}_{13}$ of the PSU was still kept above $90 \%$ (Figure 3.8c). The influence of surfactant was further studied through immobilizing the entire PSU with TWEEN 20. From Figure 3.6d we can clearly see that a large amount of RBCs migrated from layer 1 to layer 2, which indicated severely decreasing in the $\mathrm{ASE}_{12}$ and $\mathrm{ASE}_{13}$ of a PSU (Figure 3.7d). This result showed that excessive amount of surfactant can significant decrease the ASE even under the condition of salt functionalization. As a result, $20 \%$ of TWEEN 20 was the chosen as the optimal amount.

Separation yield describes the percentage of extracted plasma volume from the total volume of blood injected. In the current study, we introduced the concept of ASY for judging the PSU performances. The ASY was calculated according to Equation (3) below:

ASY $=1$-(weight of RBCs absorbed on membrane ( $\mathrm{g}$ )/weight of $30 \mu \mathrm{L}$ of blood sample (g))

The weight of RBCs absorbed on membrane was calculated according to the weight difference of membrane layer-1 before and after blood plasma separation. Figure 3.8 describes the ASYs of six PSUs when $30 \mu \mathrm{L}$ of human blood sample is loaded on each PSU. From the figure we can see that the ASYs of PSUs approximated 30\%, which indicated that the PSU was capable of separating plasma from blood samples with outstanding efficiency and stability, following the following PLFS tests with dramatically reduced interference from RBCs.

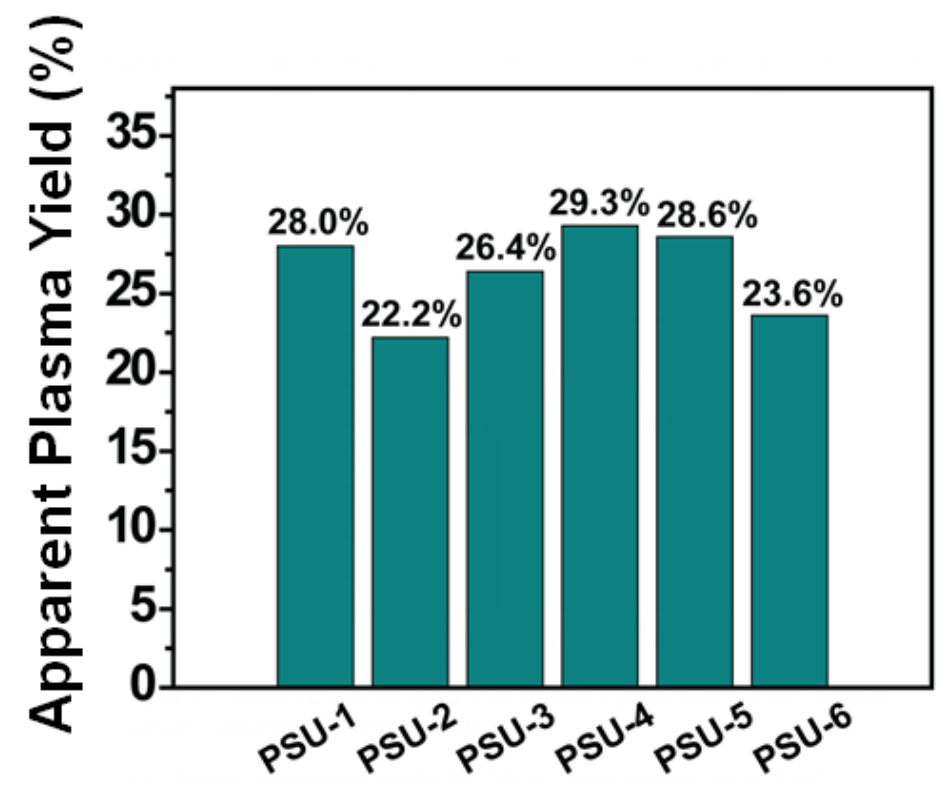

Figure 3.8. ASY of PSUs when $30 \mu \mathrm{L}$ of human blood sample was loaded. The test of blood plasma separation was repeated six times on six PSUs. 


\subsubsection{Analytical Characterization in Whole Human Blood}

In order to evaluate the performances of the PSU-PLFS, whole blood sample solutions containing different concentrations of CEA (ranging from 0 to $100 \mathrm{ng} / \mathrm{mL}$ ) were measured under optimal experimental conditions. During a typical test, $30 \mu \mathrm{L}$ of blood sample was loaded on the first filter layer of the PSU. After blood plasma separation, RBCs were captured inside of the PSU, whereas plasma containing target CEA was wicked by capillary force into the PLFS, which was functionalized with detection and capture reagents for CEA measurement. In order to improve detection sensitivity, $100 \mu \mathrm{L}$ of PBS running buffer was then dropped onto the second filter layer of the PSU, which was used to drive the rest CEA molecules to migrate to the detection zone on the PLFS. In addition, buffer introduction can also reduce non-specific absorption on the detection area. As descripted in Figure 3.9a, the test line intensity increases with the increasing of CEA concentration in blood samples. Moreover, most of the RBCs are intercepted inside of the PSU; while the detection area of the PLFSs are not contaminated by RBCs. This result indicates that the PSU-PLFS is capable of directly sensing target biomolecules from whole blood samples. Figure 3.9b and $\mathbf{c}$ respectively exhibits the SERS spectra obtained from the PLFS and the corresponding calibration curve. The regression equation was $y=1158.5 x$ -239.0 with a correlation coefficient of 0.98 . The detection limit of target CEA was found to be $1 \mathrm{ng} / \mathrm{mL}$, which was estimated based on three times of signal-to-noise ratio. A linear detection range from $5 \mathrm{ng} / \mathrm{mL}$ to $100 \mathrm{ng} / \mathrm{mL}$ was obtained and was suitable for quantitative work. The above results implied that the developed PSU-PLFSs were working sensitively and reliably, which attributes both to the high separation efficiency of the PSU and also to the high stability and sensitivity of the sandwich-typed SERS probes in the complex human fluids. 
a)

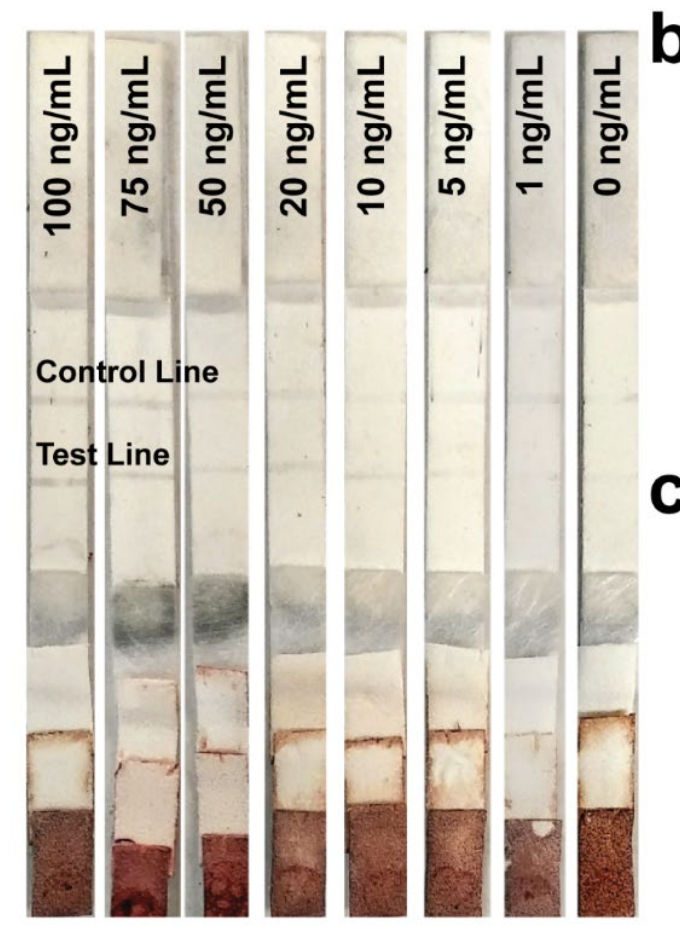

b)
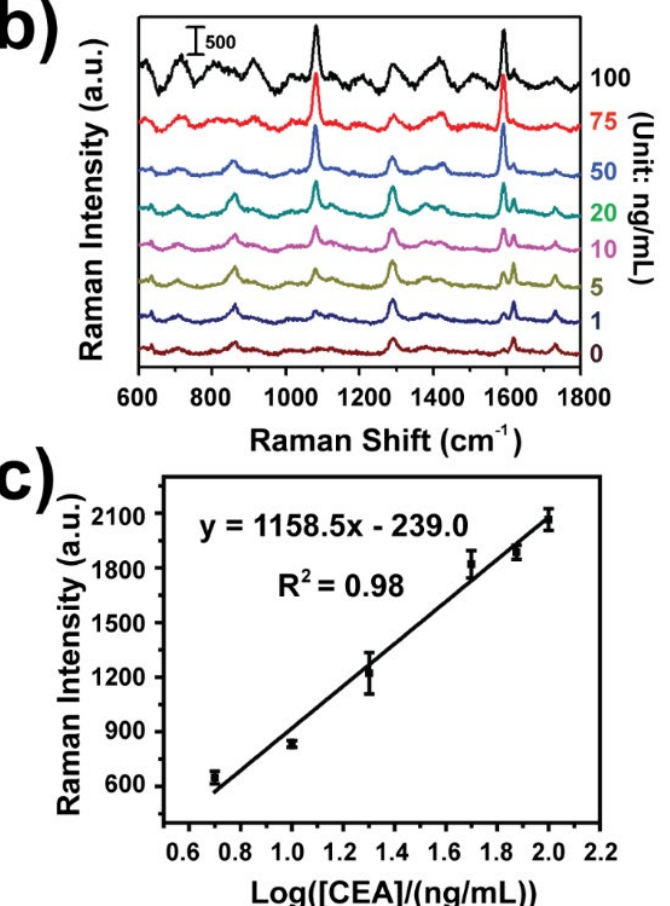

Figure 3.9. Optical images (a), SERS spectra (b) and the corresponding calibration curve of the PSU-PLFSs for CEA detection in whole blood samples.

\section{5. CONCLUSION}

A PSU has been developed for separating blood plasma from whole blood samples with high separation efficiency and separation yield. The outstanding performance of the PSU was achieved through employing $\mathrm{NaCl}$ and TWEEN 20 functionalizations on blood filtration membranes. The treatment of the $\mathrm{NaCl}$ solution to first and third filter layers guaranteed that the RBCs can be firmly intercepted inside of the PSU; while the TWEEN 20 treatment helped accelerate separating plasma from the sticky whole blood sample. The PSU was then integrated into a PLFS for cancer protein biomarker detection. A LOD of $1 \mathrm{ng} / \mathrm{mL}$ of CEA was obtained by the PSU-PLFS from whole blood samples. The high sensitivity was attributed to the reduced interferences from whole blood and the improved stability of the SERS probe in human fluids. Moreover, the fabricated PSU can be employed in various paper devices to reduce the RBCs interferences, showing great potential in the POC settings in remote and developing regions. 


\section{Chapter 4: PLASMONIC CHIP-TEST STRIP IN WHOLE BLOOD TESTING}

\subsection{NANOSTRUCTURED CHIP INTEGRATED TEST STRI P}

The demands of point-of-care testing (POCT) have steadily risen during the past half century. The global cost of POCTs was valued at USD 17.6 billion in 2018 and is expected to reach a value of 36.2 billion by the end of 2025. The features of an ideal POC device are aimed at delivering user-friendly, cost-effective and robust care near patient's home. ${ }^{51}$ Among POC devices, capillary flow devices are the most widely distributed and employed forms. ${ }^{52}$ The two representative examples are pregnancy test strips and glucose test strips. The prevalence is owing to that they are portable and user friendly, which is suitable for timely self-testing without any professional training. Moreover, the components of test strips are made from papers, which are low-cost and facilely storable, enabling multiple-time screenings over a specific period. The underlying mechanism of a test strip is mainly based on sensing of a target molecule or a biomarker. For example, a pregnancy test strip is manufactured for detecting human chorionic gonadotrophin $(\mathrm{hCG})$ in urine samples. If the $\mathrm{hCG}$ level attains the detectable limit of a pregnant test strip, the optical signal from the captured bioprobe linked Gold nanoparticles (GNPs) can be observed and used for signal readout. However, as a POC tool, there are tradeoffs. In order to simplifying test procedures and accelerating sample screening, sensitivity and accuracy of a test strip are sacrificed. As a result, most of the current test strips cannot be applied for early-stage biomarker sensing, owing to that early-stage diseases always relate with trace-amount of biomarkers. Therefore, it is of great significant to develop POC tools with enhanced performances to address the unmet need for sensitive and reliable measurement of early-stage biomarker, which is beneficial to providing timely diagnosis and properly management and finally increasing patient survival rates.

Optical and electrochemical techniques have been widely employed in test strips. Sensing signals are generated through interfacing biological recognition events in test strips and then transmitted through transducers to a signal processor for data analysis. Integrating nanomaterials into test strips is an effective way to improve detection sensitivity. ${ }^{52}$ It is because these nanomaterials possess unique properties, such as inherent large surface area, excellent 
biocompatibility, outstanding conductivity and catalytic ability, which help amplify the transduction of biorecognition events, retain bioactivity of biomolecules and accelerate signal transduction, leading to an elevation in detection sensitivity. GNP is the most representative signal tag that used in test strips, owing to the fact that GNPs are stable, easy to synthesize and can provide strong contrast with the white background of a test strip. Moreover, the optical properties of GNPs can be easily tuned through changing their size and morphologies, which helps amplify response signal. Besides GNPs, silver nanoparticles, ${ }^{53}$ magnetic nanoparticles, ${ }^{54}$ fluorescent based quantum dots and organic dyes and fluorescent nanoparticles ${ }^{55}$ have also been employed as signal reporters to elevate the performances of test strips. However, most of the above mentioned sensors fail in real-world sample applications due to non-specific interferences and strong color interference from sample matrices, especially from whole blood samples. In order to overcome this issue, sample pre-treatment, sample dilutions and introducing signal amplification techniques are always required. As a result, the involved complicate experimental procedures limit their applications in POC uses.

Surface-enhanced Raman scattering (SERS) is a powerful technique that possesses ultrasensitivity by amplifying the Raman scattering signal by orders of magnitude if a molecule is associated to the surface of a rough metal or plasmonic nanomaterial. ${ }^{56}$ This improvement is based on the coupling of EM field enhancement of nearby noble metal nanomaterials, which leads to the formation of "hot spots" for SERS enhancement. The tremendous enhanced Raman responses make SERS technique attractive for ultrasensitive sensing. ${ }^{57}$ In addition, SERS technique owns "fingerprint" feature to elevating the anti-interference resistance to nonspecific molecule, which is well-suited for specifically targeting biomolecules from complex sample matrices. Gold and silver are the most representative SERS-active nanomaterials. They can be integrated into sensors as nanoparticles, patterned nanostructures or sharp metal tips. In order to maximize the effect of enhancement, the size and morphology of SERS-active nanomaterials have been precisely controlled. Recently, our group developed a three-dimensional (3D) plasmonic nano-architecture immunosensor for cancer biomarker sensing. ${ }^{40}$ A $3 \mathrm{D}$ confined plamonic field was created by coupling the gold triangle nanoarray with the sandwich-typed SERS probe, which generated a high density of "hot spot" for ultrasensitive detection. In addition, the gold triangle nanoarray chip can concentrate the plasmonic field and create hot spots near the sharp tips, leading to dramatically improvement in SERS signal and detection 
sensitivity. As a result, this immunosensor was capable of detecting cancer protein biomarker directly in clinical blood plasma samples. Although the immunosensor exhibited advantages of shorter detection time compared with the standard ELISA method, it is difficult to be utilized as a POC tool, since expert personnel is still required to operate the test.

In the current study, we designed a 3D plasmonic nano-architecture functionalized lateral flow test strip, which can sustain the property of a POC tool, at the same time, realize ultrasensitive measurement of biomolecules in complex human fluids. Similar with the above mentioned gold triangle nanoarray chip, the proposed gold nanopyramid array chip in this study also presents sharp tips, which is expected to provide high sensitivity for SERS sensing. During the device fabrication, the capture antibody was immobilized on the chip surface; while the SERS gold nanostar@Raman molecules@silica sandwich nanoparticles were labeled with detection antibody. In the present of target molecule, the detection antibody linked SERS nanoparticles are captured over the gold pyramid nanoarray, forming a confined 3D plasmonic field, leading to the significantly enhanced electromagnetic field in intensity and in 3D space. During the detection, no extra washing operations were required owing to the present of a surfactant bridge proposed in this study. The surfactant bridge was built across the chip surface to reduce the surface tension, which ensures the sample fluid can flow smoothly across the entire device. Compared with the signal from SERS nanoparticles on NC membrane, the SERR signal from the 3D plasmonic nano-architecture was improved significantly. As a result, the developed

plasmonic chip integrated test strip was capable of detecting TBI protein biomarker directly from the plasma samples of clinical patients, showing great promises for detection of biomarkers in POC applications.

\subsection{MATERIALS AND METHODS}

\subsubsection{Reagents and Materials}

4-Mercaptobenzoic acid (technical grade, 90\%) (4-MBA) was purchased from Sigma-Aldrich. 3-Triethoxylsilylpropyl succinic anhydride (TEPSA) was obtained from Gelest Inc. Nhydroxysuccinimide (NHS), 1-ethyl-3-(3-(dimethylamino)-propyl) carbodiimide (EDC), 11mercaptoundecanoic acid (MUA), 11-mercapto-1-undecanol ethanolic (MU) and tetraethyl orthosilicate (TEOS) were purchased from Sigma-Aldrich; $\mathrm{HAuCl}_{4}$, sucrose, $\mathrm{Na}_{3} \mathrm{PO}_{4} \cdot 12 \mathrm{H}_{2} \mathrm{O}$, Tween 20, Triton $\mathrm{X}-100$, trisodium citrate, $\mathrm{PBS}(\mathrm{pH}=7.4), \mathrm{Na}_{3} \mathrm{PO}_{4} \cdot 12 \mathrm{H}_{2} \mathrm{O}$, BSA (bovine serum 
albumin) and sodium chloride were purchased from Sigma-Aldrich and used without further purification. Sucrose was purchased from VWR. S-100 $\beta$ and anti-S-100 $\beta$ antibody were procured from Sigma-Aldrich. S-100 $\beta$ ELISA Kits were purchased from R\&D System. The blood plasma was purchased from US Biological Life Science (P4252-56 Plasma, Human, IgG Free). Glass fibers (GFCP000800), cellulose fiber sample pads (CFSP001700), laminated cards with Hi Flow plus cards (HF090MC100 with RK12312 Membrane $(0.45 \mu \mathrm{m})$ ), and nitrocellulose membrane (RK13464 $(0.22 \mu \mathrm{m})$ ) were purchased from Millipore (Billerica, MA). Quartz slides were purchased from Fisher Scientific Inc. Polystyrene (PS) beads (500nm diameter) were purchased from Thermo Scientific. Clinical blood plasma samples were collected from TBI patients and provided by WVU medical school.

\subsubsection{Instruments and Characterization}

All Raman spectra were acquired with a portable B\&W Tek i-Raman Plus. Fourier transform infrared (FT-IR) spectra were obtained under the attenuated total reflection (ATR) mode with Thermo Nicolet 6700 spectrometer. The nanoparticles were characterized with a transmission electron microscope (TEM, JEOL JEM-2100F) at an acceleration voltage of $200 \mathrm{kV}$. UV-visible absorption spectra were obtained with a Shimadzu UV-2550 spectrometer. Titanium and gold metals were deposited by e-beam evaporator (Kurt J Lesker). A scanning electron microscope (JEOL-JSM-7600F) was used to image the gold nanopyramid array. Optical characterization of gold nanopyramid array was done using an Ocean Optics 4000 spectrometer and DT-MINI-2GS.

\subsection{PREPARATION PROCEDURES}

\subsubsection{Synthesis of Gold Nanopyramid Array Chip and Functionalization of Capture Antibody on} Chip

Nanosphere lithography fabrication technique was used to fabricate the nanopyramid structure. Initially quartz slides were cut in the form of chips with dimensions around $1 \mathrm{~cm} \mathrm{x}$ $1 \mathrm{~cm}$. The chips were cleaned in acetone, methanol and DI water for $5 \mathrm{~min}$ in each respectively. The cleaned quartz chips were immersed in acid piranha at $90{ }^{\circ} \mathrm{C}$ for $1 \mathrm{~h}$, which makes the quartz surface hydrophilic. Further, the chips were rinsed in DI water and 500nm diameter polystyrene (PS) beads were dip-coated to form a Hexagonally Closed Pack (HCP) monolayer. The monolayer is allowed to dry for $10 \mathrm{~min}$. Later, a $5 \mathrm{~nm}$ titanium film was deposited followed by 
$250 \mathrm{~nm}$ gold film using e-beam evaporator. The metal deposits on to the quartz through the interstitials spaces of nanospheres to form pyramid shape. The polystyrene beads were removed by ultrasonication of chips in methanol, leaving the gold nanopyramids on the quartz substrate. Due to the titanium adhesive layer, the gold nanopyramids were intact on the quartz substrate.

The fabricated chip was cleaned by successive immersion in ethanol and D.I. water for 10 min, respectively. The cleaned gold films were incubated overnight in a solution containing 100 $\mathrm{mM}$ MUA/100 $\mathrm{mM}$ MU and then washed using ethanol and D.I. water, respectively. The successful conjugation of MUA/MU onto the gold film was confirmed by FT-IR spectra (Figure 4.1). The resulting MUA/MU modified chip was activated by immersion in a solution containing $50 \mathrm{mM}$ NHS/200 mM EDC in a PBS solution. The chip was incubated overnight in a PBS solution containing $1 \mathrm{mg} / \mathrm{mL}$ of anti-S-100ß. Successful conjugation of capture antibody onto the chip was confirmed by FT-IR spectra (Figure 4.2), in which the peak at $1541 \mathrm{~cm}^{-1}$ indicated the $\mathrm{N}-\mathrm{H}$ bending. After immobilization of the antibody, the chip was successively rinsed with D.I. water and PBS solution to remove free and excess antibody molecules.

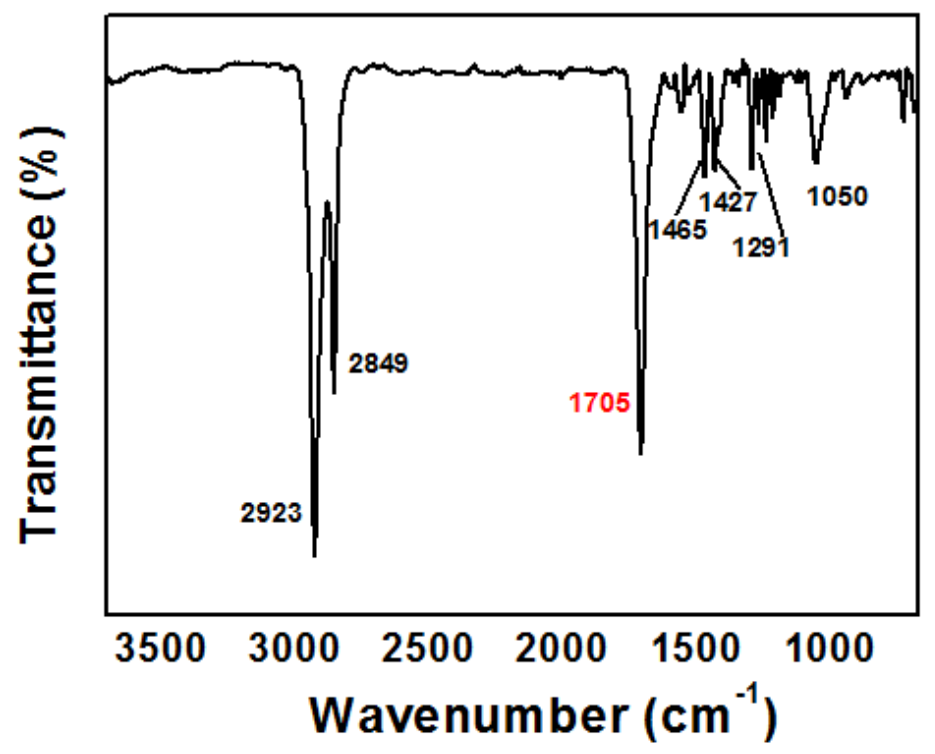

Figure 4.1. FT-IR spectra of MU/MUA functionalized chip. The peak at $1705 \mathrm{~cm}^{-1}$ peak is assigned to the $\mathrm{C}=\mathrm{O}$ stretching vibration. 


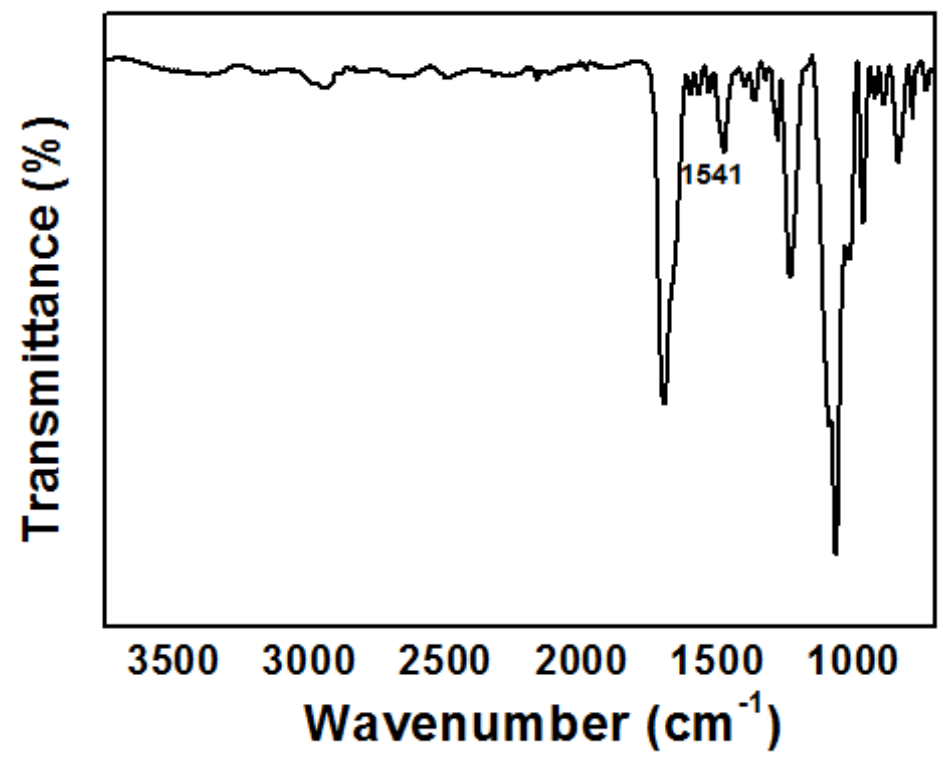

Figure 4.2. FT-IR spectra of the capture antibody functionalized chip. The peak at $1541 \mathrm{~cm}^{-1}$ corresponds to the $\mathrm{N}-\mathrm{H}$ bending in the antibody.

\subsubsection{Functionalization of Surfactant Bridge on Chip Surface}

The surfactant bridge contains two components: TWEEN 20 and glucose. $1 \mu \mathrm{L}$ of TWEEN 20 was dropped on and then spread evenly on the surface of the capture antibody immobilized plasmonic chip. After that $0.01 \mathrm{~g}$ of glucose was sprinkled on the TWEEN 20 covered chip surface. The surfactant bridge functionalized chip was stored in dry ambient for $0.5 \mathrm{~h}$ and then stored at $4^{\circ} \mathrm{C}$ for further use.

\subsubsection{Synthesis of Nanoparticles and Conjugation with Detection Antibodies.}

The Au nanostars, the SERS probes, and the nanoparticles conjugated with detection antibodies were synthesized according to our previous paper. ${ }^{58}$ Briefly, the Au nanostar@Raman molecule@Silica sandwich SERS probes were synthesized by adding 1.0 $\mu \mathrm{M}$ Raman molecules into the $\mathrm{Au}$ nanostar suspension. Then, $200 \mu \mathrm{L}$ of $1 \%$ TEOS and $15 \mu \mathrm{L}$ of ammonium solution were added into the mixture, successively. After the SERS probes were formed, the resulting solutions were centrifuged and resolved in $500 \mu \mathrm{L}$ of ethanoic solution. The Au nanostars were coated with a 2-3 nm thick silica layer on the outer surface. Next, $100 \mu \mathrm{L}$ of TEPSA was added to the SERS probe solution and then incubated overnight to achieve the carboxyl-group terminated SERS probes. The conjugation of carboxyl-group onto the surface of SERS nanoparticles was confirmed by FT-IR spectra (Figure 4.3). The carboxyl-group terminated 
sandwich nanoparticles were then activated by adding a PBS buffer solution containing $50 \mathrm{mM}$ NHS and $200 \mathrm{mM}$ EDC and then incubated for $1 \mathrm{~h}$. Subsequently, $100 \mu \mathrm{L}$ of $1.0 \mathrm{mg} / \mathrm{mL}$ detection antibody was added, and the mixture was incubated overnight. The successful conjugation of detection antibody onto SERS nanoparticles was confirmed by the FT-IR spectra (Figure 4.4). After that, the mixture was centrifuged and washed with a PBS buffer three times to remove excessive free antibody. The resulting SERS probe conjugates were stored in $100 \mu \mathrm{L}$ of eluent buffer $\left(20 \mathrm{nM}\right.$ of $\mathrm{Na}_{3} \mathrm{PO}_{4} \cdot 12 \mathrm{H}_{2} \mathrm{O}$ containing $5 \%$ BSA, $0.25 \%$ Tween 20 , and $10 \%$ sucrose) solution for future use. The corresponding characterizations can be found from Chapter 2 in this dissertation.

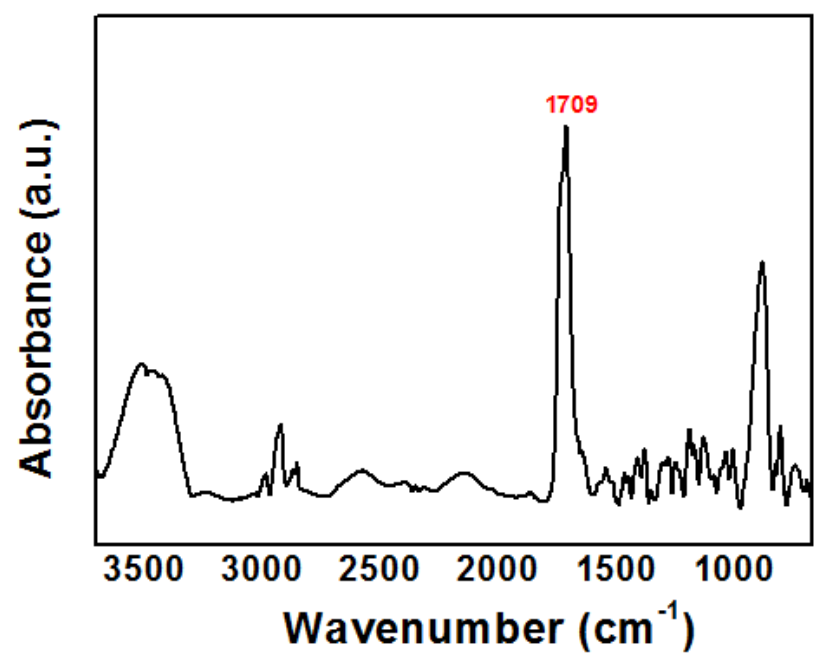

Figure 4.3. FT-IR spectra of the carboxyl-group modified SERS probe. The peak at $1709 \mathrm{~cm}^{-1}$ peak is assigned to the $\mathrm{C}=\mathrm{O}$ stretching vibration in $\mathrm{COOH}$ of TEPSA.

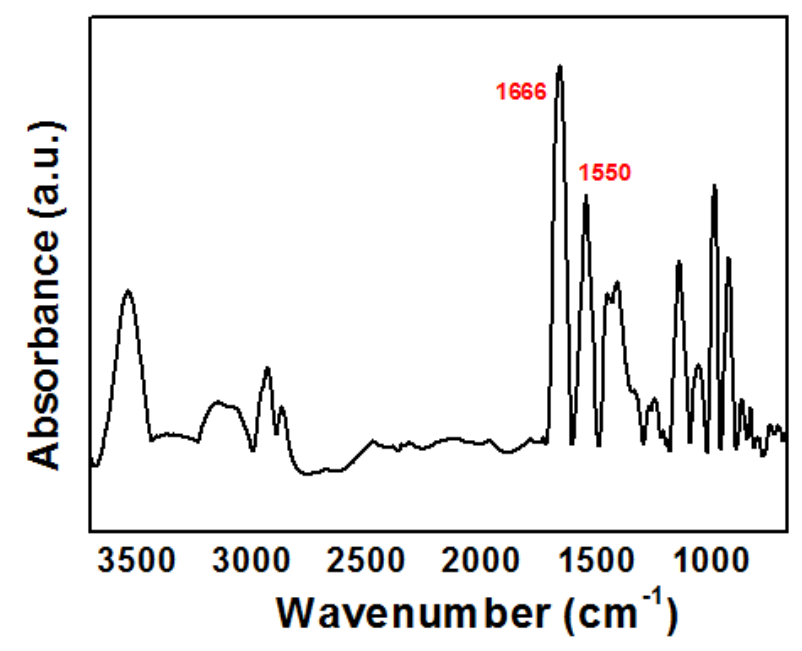


Figure 4.4. FT-IR spectra of the detection antibody functionalized SERS probe. The peaks at 1666 and $1550 \mathrm{~cm}^{-1}$ : correspond to the N-H bending in the antibody.

\subsubsection{Preparation of Chip-Test Strip}

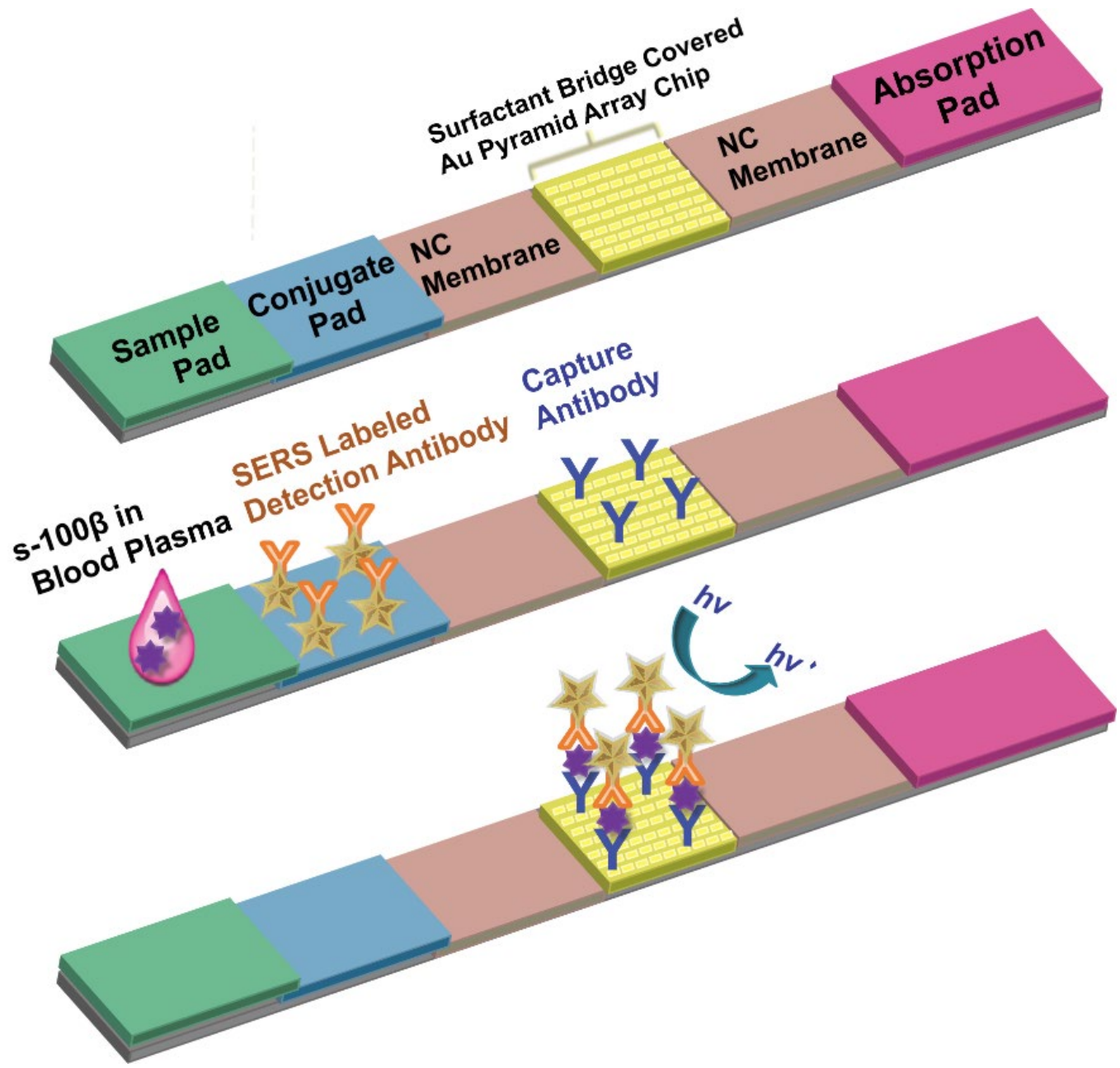

Figure 4.5. Schematic illustration of the operation principle of chip integrated PLFS.

Figure 4.5 shows the schematic diagram of the fabricated chip-PLFS, which consists of five components: a sample pad, a conjugate pad, two nitrocellulose membranes (NC membrane), a gold nanopyramid array chip and an absorption (or wick) pad. All the components were mounted on a common backing layer (typically an inert plastic, e.g., polyester). The sample pad was saturated with a Tris- $\mathrm{HCl}$ buffer $(\mathrm{pH} 8.0)$ containing $0.23 \%$ Triton $\mathrm{X}-100$ and 0.05 and $0.15 \mathrm{M}$ $\mathrm{NaCl}$. The pad was then dried at $37^{\circ} \mathrm{C}$ for $2 \mathrm{~h}$ and stored in a desiccator at room temperature (RT). Then, the surfactant bridge covered capture antibody labeled chip was placed between two 
NC membranes. The dimension of each NC membranes was $1.1 \mathrm{~cm} \times 0.3 \mathrm{~cm}$. The detection antibodies modified SERS probes were dropped onto the conjugate pad. Finally, all the five processed components were assembled onto a plastic adhesive backing layer. The paper components were overlapped with $2 \mathrm{~mm}$ to ensure that the solution was able to flow through the whole strip. Each paper component was cut to be $3 \mathrm{~mm}$ wide by a paper cutter (Swingline $12 \mathrm{in}$. ClassicCut Lite Trimmer). Before testing, $5 \mu \mathrm{L}$ of detection antibody linked SERS probes was loaded onto the conjugate pad. During testing, $100 \mu \mathrm{L}$ of sample solution containing a certain concentration of target biomolecule was dropped on the sample pad.

\subsection{RESULTS AND DISCUSSION}

\subsubsection{Characterizations of Gold Pyramid Nanoarray}

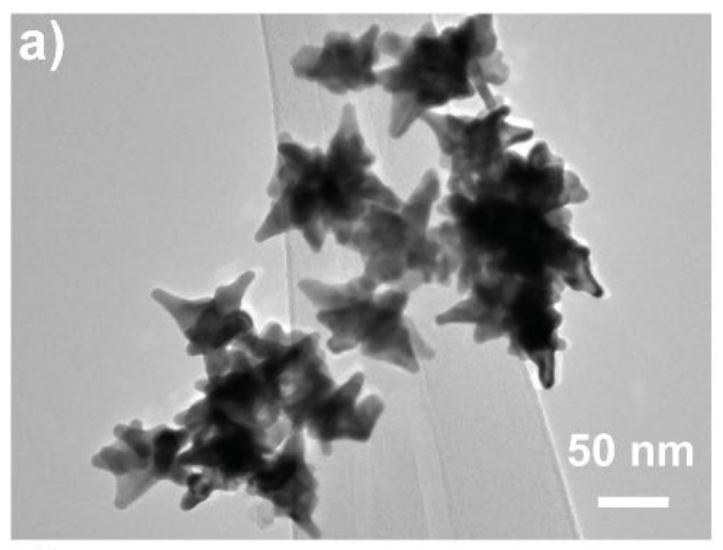

c)

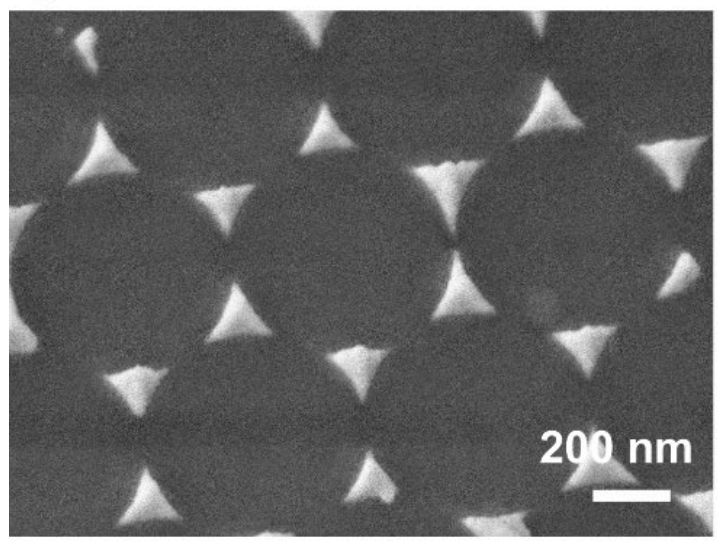

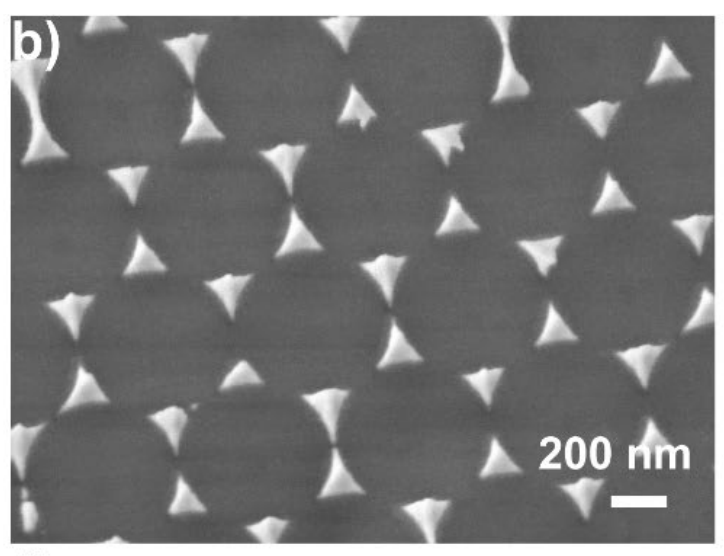

d)

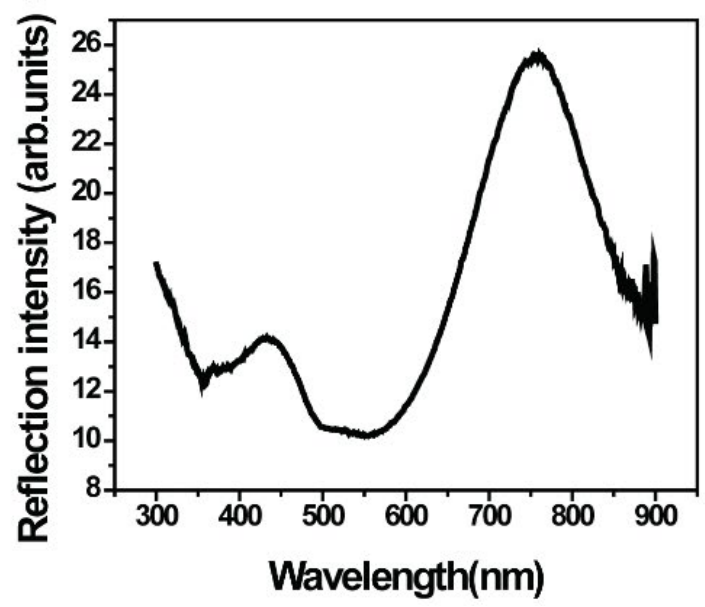

Figure 4.6. a) TEM images of the gold nanostar@MBA@ $\mathrm{SiO}_{2}$ sandwich nanoparticles; SEM images of b) large-area view and c) enlarged view of the gold nanopyramid array, and d) UV-Vis reflection spectra of the gold nanopyramid array. 
The synthesized SERS nanoparticles contain a core of gold nanostar and a silica shell; while the Raman molecules are sandwiched between the gold core and the silica shell. The metal core is able to amplify the SERS signal; while the silica shell can protect the nanoparticles away from the interferences of surrounding environment. In addition, numerous of Raman molecules can be concentrated inside of the silica shell of one single SERS probe, which can significantly improve sensitivity. Figure 4.6a, exhibits the TEM image of the gold nanostar@MBA@ $\mathrm{SiO}_{2}$ sandwich nanoparticles. It can be clearly seen that the gold nanostars exhibit a star shape and possess sharp tips, which contributes to remarkable signal amplification. A 4 5 nm thick $\mathrm{SiO}_{2}$ layer can also be observed from the TEM image, which indicates that the silica shell was successfully coated onto the nanoparticles. Figure 4.6b and $\mathbf{c}$ show the SEM images of the gold nanopyramid arrays. The gap between the adjacent pyramid corners in the gold pyramid nanoarray was about $40 \mathrm{~nm}$, which enables the coupling of LSPR. The UV-Vis reflection spectra from Figure 4.6d further confirms that the gold nanopyramid array is capable of coupling LSPR, generating a high density of hot spots for the SERS enhancement.

\subsubsection{Operation Principle}

The prepared plasmonic chip-PLFS contains dry reagents which can be activated by applying a fluid sample (Figure 4.5). Once the sample solution is dropped onto the sample pad of the chip-PLFS, it will flow automatically from sample pad to conjugate pad by capillary force. On conjugate pad, an interaction between target protein and detection antibody linked SERS probes occurs. The resulting complex continues to move along with the chip-PLFS until it reaches the boundary between the NC membrane and the plasmonic chip. With the help of surfactant bridge, the sample solution can migrate smoothly across the chip surface. At the same time, the surfactant bridge starts to dissolve and the capture antibody labeled chip surface is exposed for capturing the above obtained bio-complex. Figure 4.7 shows that the surfactant bridge is dissolved 2 min after running buffer is added, indicating that the fluid flows smoothly across the device, meanwhile the surfactant bridge doesn't block signal acquisition from chip surface. 


\section{$0 \mathrm{~min}$}

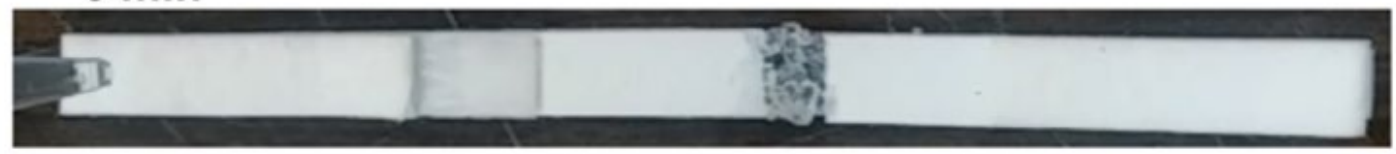

\section{$2 \mathrm{~min}$}

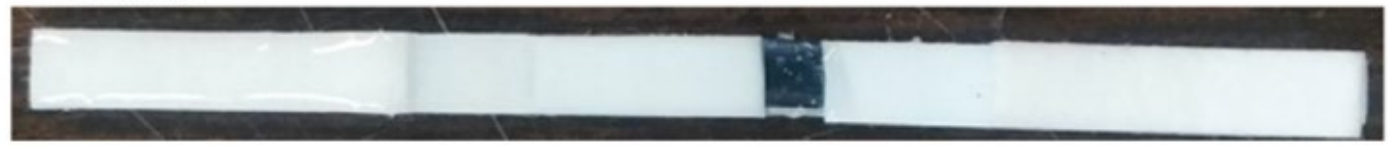

Figure 4.7. Optical images of a chip-PLFS before and after the sample solution is loaded.

As a result, a sandwich-typed structure of SERS probe/detection antibody-protein-capture antibody is formed on the plasmonic surface, leading to the formation of a confined 3D plasmonic field for generating ultrasensitive SERS signal. The operation principle is illustrated in Figure 4.8. A portable Raman spectrometer is employed to record the SERS signal from the plasmonic chip surface. Under excitation by a $785 \mathrm{~nm}$ laser, the SERS signal is generated from the MBA (Raman reporter) molecules inside of the captured SERS probes. The obtained SERS signal is in proportion to the concentration of target protein, which is used for quantitative work.

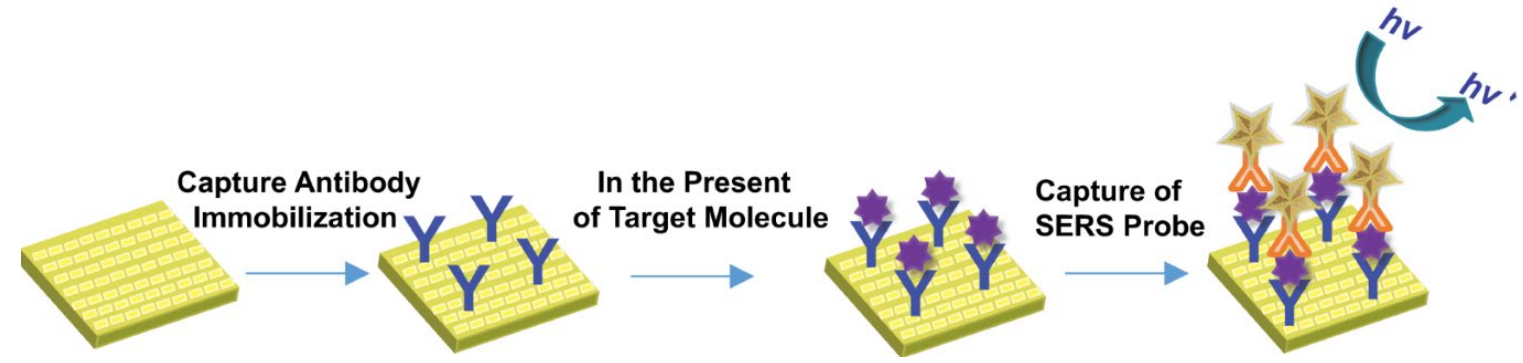

Figure 4.8. Schematic illustration of the reactions on chip integrated PLFS.

\subsubsection{Comparison of Raman Signal on Different Substrate}




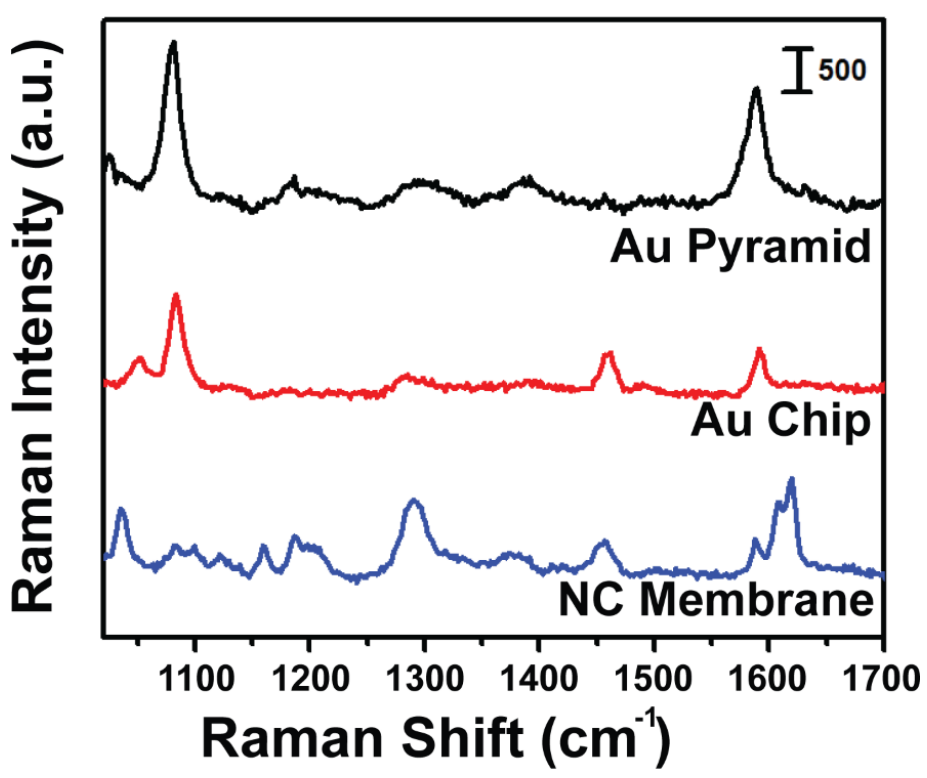

Figure 4.9. Comparison of SERS signal on Au pyramid, planar Au chip and NC membrane respectively.

In order to verify the substantial improvement in SERS signal, a comparison study was conducted. Three configurations of plasmonic hierarchical architecture were employed as SERS substrates, including SERS NPs on Au nanopyramid array chip, SERS NPs on planar Au chip and SERS NPs on NC membrane. From Figure 4.9 we can find that the SERS NPs/Au nanopyramid array chip exhibits the highest SERS signal at the specific Raman peak of $1084 \mathrm{~cm}^{-}$ 1, while the signal from SERS NPs/NC membrane is much less efficient. This signal improvement is endowed from the high density of hot spots in the 3D hierarchical architecture of the SERS NPs/Au nanopyramid array chip. In addition, both of gold nanostars are gold nanopyramid array possess LSPR bands at $\sim 785 \mathrm{~nm}$, which are overlapped with the wavelength of excitation laser, leading to dramatically improvement in sensing performance. This result also allows us to investigate the effects of the plasmonic chip (Au planar chip and Au nanopyramid array chip) and the non-plasmonic substrate (NC membrane) on the performance of the SERSPLFS.

\subsubsection{Optimizations}



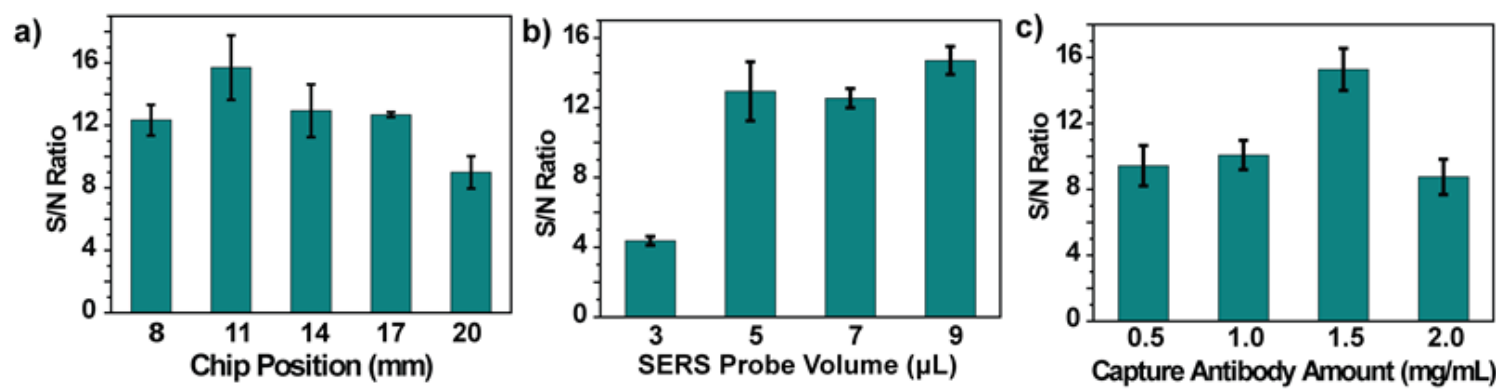

Figure 4.10. Optimization of the experiment conditions for chip-PLFS. a) Effect of the chip displaying position on the S/N ratio of chip-PLFSs, b) effect of SERS probe volume on the $\mathrm{S} / \mathrm{N}$ ratio, c) effect of effect of the amount of capture antibody modified on chip on the $\mathrm{S} / \mathrm{N}$ ratio.

In order to elevate the performance of the chip-PLFS, human IgG and anti-IgG antibody was chosen as models to optimize the device. $10 \mathrm{ng} / \mathrm{mL}$ of human $\mathrm{IgG}$ was used as target molecules in all of the following optimization experiments. The incubation time of detection antibody with target antigen, and capture antibody with target antigen significantly influences the binding efficiency, as well as the sensitivity. Therefore, the binding timing and extent need to adjusted, which can be implemented through optimizing the displaying positions of plasmonic chip in test strip. The displaying chip position means the distance between the front boundary sides of the plasmonic chip with the conjugate pad. Figure 4.10a reveals that the $\mathrm{S} / \mathrm{N}$ ratio of the SERS peak increased with the growing distance of the plasmonic chip away from the conjugate pad until the distance reached $11 \mathrm{~mm}$, which means when the distance is below of $11 \mathrm{~mm}$, the binding time between detection antibody and antigen is not enough. Further increasing the distance led to a decrease in the binding time between the detection antibody/antigen complexes with the capture antibody, since most of the biomolecules were immediately adsorbed by the wicking pad after they arrived at the chip surface. As a result, the optimal displaying distance of the chip in test was $11 \mathrm{~mm}$ away from the conjugate pad. Another two important parameters which affect the capturing ability of signal reporters are the amount of nanoparticle linked detection antibody and the concentration of capture antibody on the chip surface. From the S/N ratios (Figure 4.10b and c), the optimal concentration of SERS probe was $5 \mu \mathrm{L}$; while $1.5 \mathrm{mg} / \mathrm{mL}$ of capture antibody was the best amount.

\subsubsection{Measurement of S-100ß in Buffer}



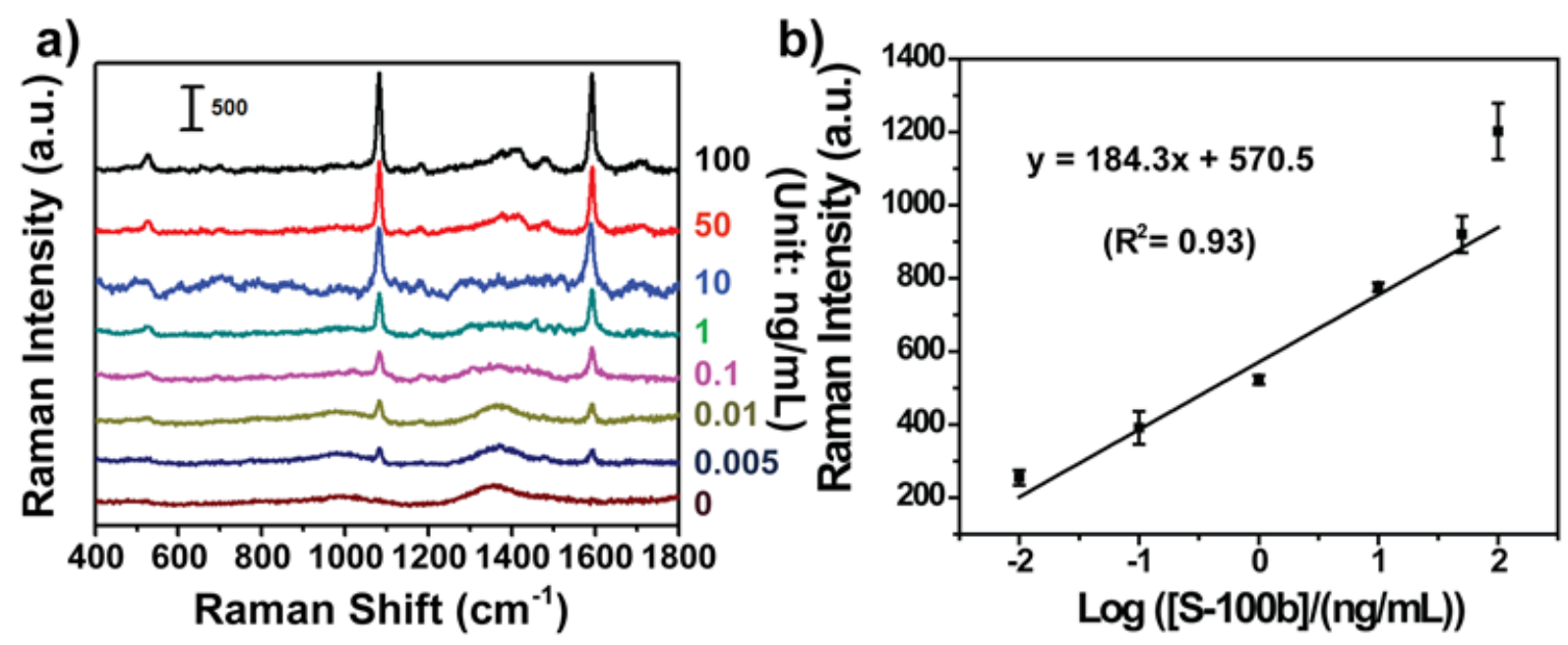

Figure 4.11. SERS spectra (a) and calibration curve (b) of chip-PLFSs with MBA as Raman reporter for detection of s-100 $\beta$ in PBS buffer.

In order to evaluate the performances of the chip-PLFS, buffer solutions containing different concentrations of s-100 $\beta$ (ranging from $0 \mathrm{ng} / \mathrm{mL}$ to $100 \mathrm{ng} / \mathrm{mL}$ ) were measured under optimal experimental conditions. During a typical test, $100 \mu \mathrm{L}$ of sample solution was loaded on the sample pad of the chip-PLFS. After 30 min reaction, the SERS signal of the chip-PLFS was recorded by a portable Raman spectrometry. Figure 4.11a and b respectively exhibits the SERS spectra obtained from the plasmonic chip and the corresponding calibration curve. The calibration curve was obtained by plotting the SERS peak intensity at $1084 \mathrm{~cm}^{-1}$ from MBA as a function of the logarithmic concentration of s-100 $\beta$. The regression equation was $y=184.3 x+$ 570.5 with a correlation coefficient of 0.93 . The detection limit of this device for s-100 $\beta$ in running buffer was found to be $1 \mathrm{pg} / \mathrm{mL}$, which was estimated based on three times of signal-tonoise ratio. Compared with the results from the previously reported SERS-test strips, the proposed chip-PLFSs exhibited a 20 times improvement in detection sensitivity, which is endowed from the high density of hot spots in the 3D hierarchical architecture. In addition, both gold nanostars are gold nanopyramid array possess LSPR bands at $\sim 785 \mathrm{~nm}$, which are overlapped with the wavelength of excitation laser, leading to dramatically improvement in sensing performance. A linear detection range from $10 \mathrm{pg} / \mathrm{mL}$ to $100 \mathrm{ng} / \mathrm{mL}$ was obtained and was suitable for quantitative work.

\subsubsection{Measurement of $s-100 \beta$ in Clinical Blood Plasma Samples}


The above results indicated that the developed chip-PLFSs were working sensitively and properly. In order to further verify the accuracy of the device, chip-PLFSs were employed to examine the $s-100 \beta$ level from plasma samples taken from six TBI patients. Commercial standard ELISA Kit was used as a comparison. Before testing clinical samples, the chip-PLFSs

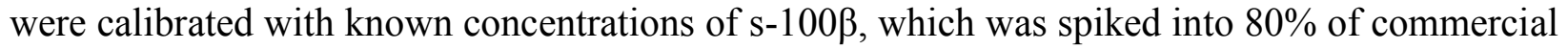
blood plasma respectively (P4252-56 Plasma, Human, IgG Free). The baseline concentration of s- $100 \beta$ in blood plasma matrices was estimated by an s-100 $\beta$ ELISA kit. No obvious signal can be observed from the blood plasma sample, which indicates that the baseline concentration of s$100 \beta$ in blood plasma is below the detection limit of the ELISA kit, showing no interference on s- $100 \beta$ sensing.
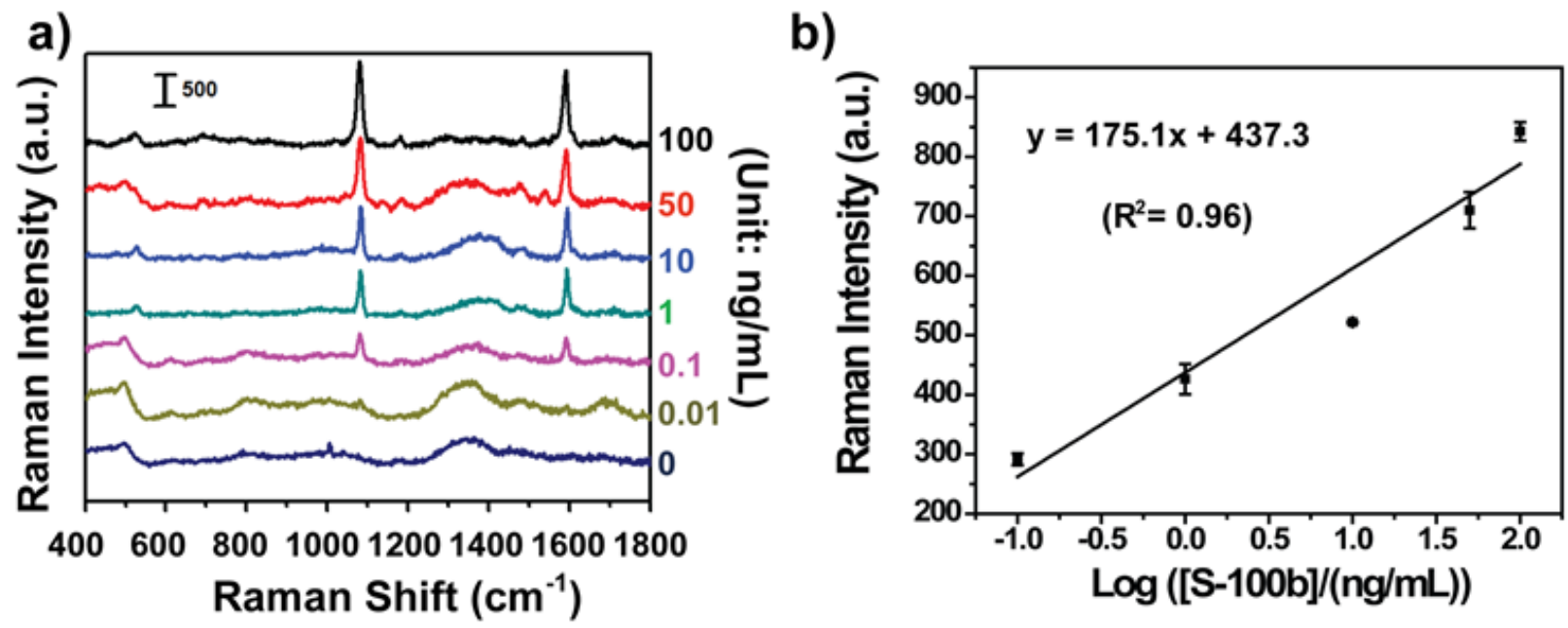

Figure 4.12. SERS spectra (a) and calibration curve (b) of chip-PLFSs for detection of s-100 $\beta$ in $80 \%$ of blood plasma.

Figure 4.12 shows the SERS spectra of the 3D hierarchical architecture integrated test strips in the presence of various concentrations of s-100 $\beta$ in $80 \%$ blood plasma. From the SERS spectra we can see that the intensity of the SERS peak at $1084 \mathrm{~cm}^{-1}$ gradually increased with an increase in the s-100 $\beta$ concentration. The SERS peak intensity (y) versus the s-100 $\beta$ concentration $(\mathrm{x})$ was fitted as $y=175.1 x+437.3$ with the relative coefficient $\left(R^{2}\right)$ of 0.96 , showing a linear detection range from 0.1 to $100 \mathrm{ng} / \mathrm{mL}$, achieving a LOD of $5 \mathrm{pg} / \mathrm{mL}$. The calibration curve indicated that the developed chip-PLFSs can work for s-100 $\beta$ detection in blood plasma. After calibration, the developed chip-PLFSs were employed for detection of s-100 
$\beta$ from clinical samples of patients with TBI. The clinical samples were also examined by the standard ELISA method. Figure 4.13 exhibits the result comparison from the chip-PLFSs and the ELISA kit. The results showed that the results from chip-PLFSs were comparable from the ones from ELISA kits, suggesting that the proposed chip-PLFSs were capable of sensing s-100 $\beta$ in clinical samples. Moreover, compared with ELISA kit, the proposed chip-PLFSs provide significant advantages, in terms of shorter sensing time (30 min vs several hours), simplified test procedures and inexpensive test.

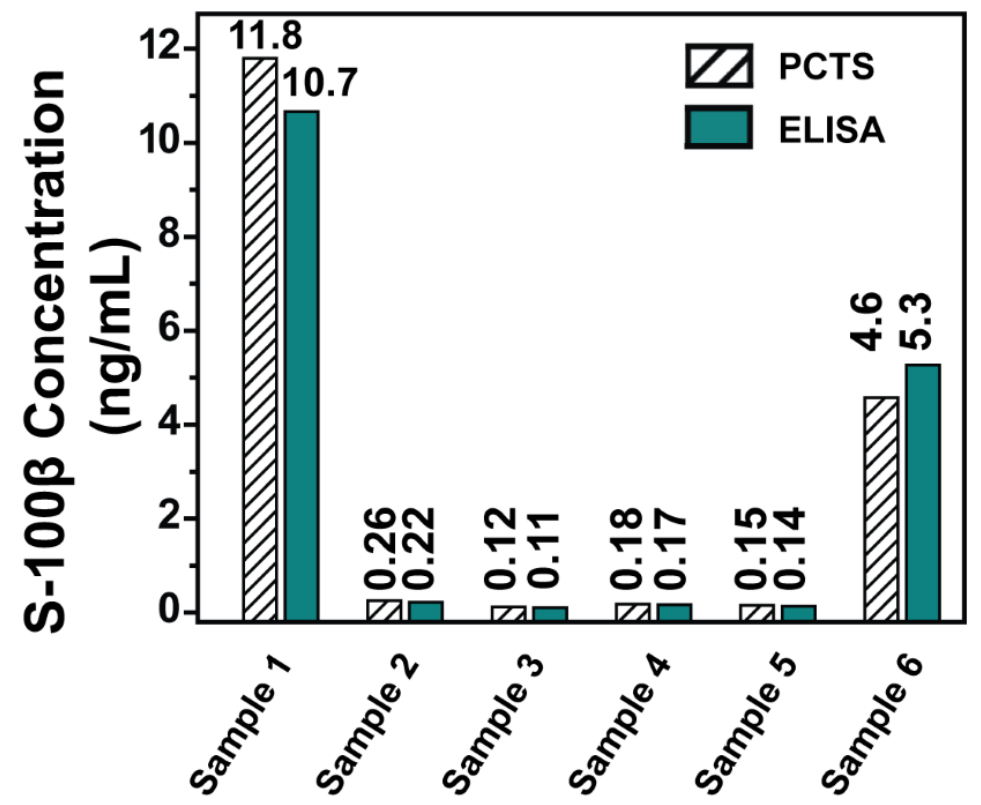

Figure 4.13. S-100 $\beta$ concentrations in clinical blood plasma samples, which were measured by ELISA and the chip-PLFS, respectively.

\subsubsection{Measurement of $s-100 \beta$ in Whole Blood Samples}

In Chapter 3, we successfully detected target protein from whole blood samples by employing a PSU integrated paper test strip. In the current study, in order to further verify the performances of the chip-PLFSs, we challenged to test s-100 $\beta$ from whole blood samples through modifying a PSU into the developed chip-PLFS. During a typical assay, $30 \mu \mathrm{L}$ of blood sample solution containing different concentrations of s-100 $\beta$ (ranging from 0 to $100 \mathrm{ng} / \mathrm{mL}$ ) was loaded on the first filter layer of the PSU. After blood plasma separation, RBCs were blocked inside of the PSU, while the separated plasma containing target s-100 $\beta$ was wicked into the chip-PLFS, which was functionalized with capture and detection reagents for s-100 $\beta$ 
measurement. $100 \mu \mathrm{L}$ of PBS running buffer was then dropped onto the second filter layer of the PSU, which was used to drive the rest s-100 $\beta$ molecules to migrate to the detection zone on the chip-PLFS; meanwhile, buffer introduction can also reduce non-specific absorption on the detection area. As descripted by the SERS spectra in Figure 4.14a, the intensity of the typical Raman peak at $1080 \mathrm{~cm}^{-1}$ increases with the increasing of $\mathrm{s}-100 \beta$ concentration in blood samples, which indicates that the PSU integrated chip-PLFSs are working properly in whole blood samples. Figure 4.14b shows the corresponding calibration curve. The regression equation was $y=20.7 x+578.9$ with a correlation coefficient of 0.95 . The detection limit of this device for target s- $100 \beta$ was found to be $50 \mathrm{pg} / \mathrm{mL}$, which was estimated based on three times of signalto-noise ratio. A linear detection range from $0.1 \mathrm{ng} / \mathrm{mL}$ to $100 \mathrm{ng} / \mathrm{mL}$ was obtained and was suitable for quantitative work. The above results implied that the developed PSU-chip-PLFSs were working sensitively and reliably, which attributes both to the high separation efficiency of the PSU and also to the high sensitivity and anti-interference ability of the developed 3D plasmonic device.
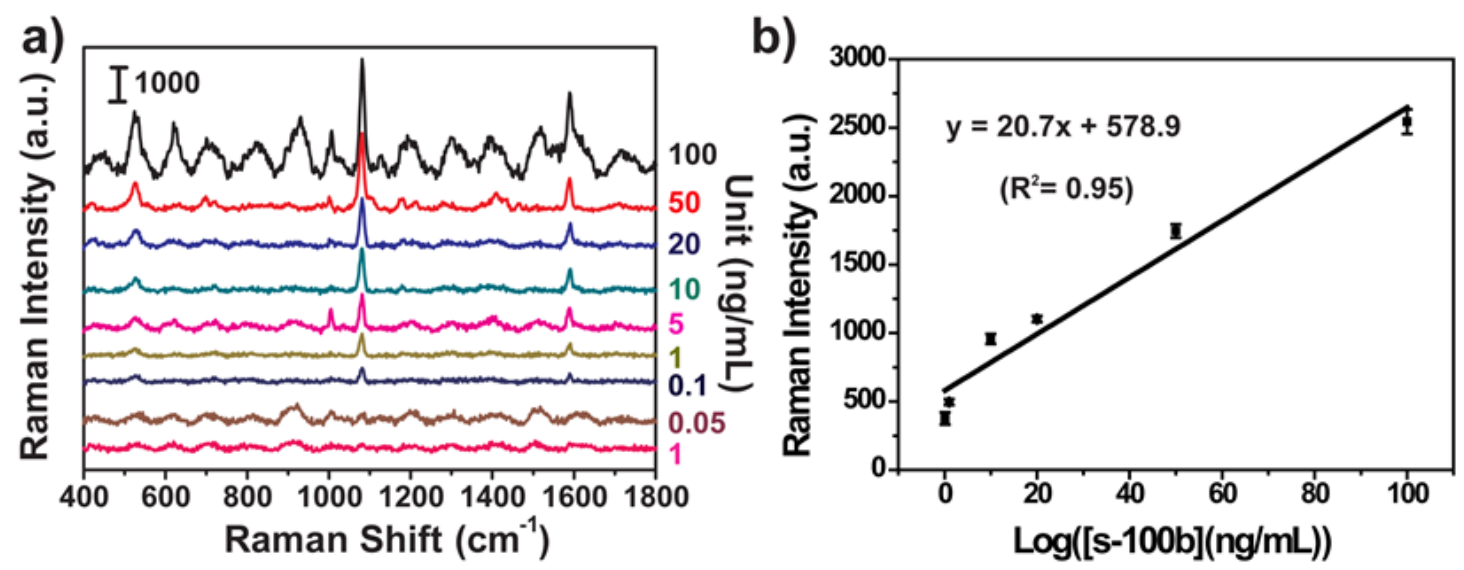

Figure 4.14. SERS spectra a) and calibration curve b) of PSU-Chip-PLFSs with MBA as Raman reporter for detection of s-100 $\beta$ in whole blood samples

\subsection{CONCLUSION}

In summary, an ultrasensitive plasmonic chip integrated test strip was developed for protein biomarker detection. In the presence of target protein, a 3D hierarchical architecture can be formed on sensing area, which forms a 3D confined plasmonic field to generating high densities 
of hot spots, leading to a significant enhancement in SERS signal. As a result, the developed chip-PLFSs showed a 20-time improvement in detection sensitivity for target protein in running buffer compared with the results from the conventional SERS test strip. In addition, the chipPLFSs possessed anti-interference ability towards complex human fluids and were capable of sensing s-100 $\beta$ directly in clinical blood plasma samples and whole blood samples. The test results were comparable with that from the standard ELISA method, showing great promises as a POC tool in real applications. 


\section{Chapter 5: NEAR-INFRARED FLUORESCENCE IN BLOOD PLASMA TESTING}

\subsection{CIRCULATING BIOMARKER: MIRNA-34}

Circulating microRNAs (miRNAs) are a class of cell-free miRNAs, which are stably present in the body fluids of many mammalian including human beings. ${ }^{59}$ Since the first description in the 1940 s, these circulating nucleic acids were found to exhibit disease-related alternations. ${ }^{60}$ For example, increased levels of plasma circulating nucleic acids have been detected in a number of diseases even at a very early stage, such as cancers, stroke and autoimmune disease. ${ }^{61}$ Circulating miRNAs are existing in many human fluids, such as serum, plasma, saliva, urine and bronchoalveolar lavage fluid, which provides diverse sensing pathways in a less-invasive mode. In addition, compared with detecting a low abundant of other biomarkers (e.g. proteins or tumor tissues), high sensitivity can be relatively feasibly achieved through detecting circulating miRNAs by the powerful qRT-PCR and microarray techniques. The unique features of circulating miRNAs, including high sensitivity, specificity and less-invasive manner expand their clinical importance in diseases diagnosis and monitoring. ${ }^{62}$ Up to now, around 100 circulating miRNAs have been identified as biomarkers which can reflect the early existence and the progression status of diseases. ${ }^{63}$ However, in order to detect circulating miRNAs with the current technologies, the extraction and purification procedures of circulating miRNAs from these human fluids are required. Although the demands of high specificity and sensitivity can be fulfilled, the complicated procedures and sophisticated instruments hindered these techniques to be operated only under the controlled laboratory environment.

Fluorescence techniques have been extensively applied in biological sensing, cell screening and clinical diagnosis owing to their low detection of limit and ability of visualization of events related disease progression with short-time data acquisition. ${ }^{27 a, 27 b, 64}$ The performances of fluorescence techniques are largely dependent on the properties of the employed fluorophores. When choosing or designing a fluorophore, several aspects should be taken into consideration, including excitation and emission nature, luminescence intensity, photostability and biocompatibility. The use of near-infrared fluorophores (NIRF) is a promising approach for biomedical sensing in biological specimens. It can reduce the damage of biological samples and 
avoid the photobleaching and background interference when visible fluorophores (VisF) are utilized. This is because that due to reduced absorbance and minimal scattering, the NIR radiation possesses high penetration ability towards tissues and biological matrices. ${ }^{29,} 65$ Meanwhile, the NIR wavelengths falls within the biological windows with low background fluorescence signals, which provides elevated signal-to-noise ratio. ${ }^{30}$ These unique features offer great opportunity to employ NIRFs in biological fields. Nevertheless, in contrast to VisFs, the major shortcoming of NIRFs is limited intense. Additionally, its biocompatibility and photostability should also be concerned during the design of a NIRF probe.

Dye-encapsulated silica nanoparticles appear to be an effective alternative to achieve the goals. The superior properties of such nanoparticles include amplified signal, outstanding photostability, water-solubility and viability of surface modification. ${ }^{31,66}$ For example, the silica layer enables a large number of dye molecules to be encapsulated inside of one single silica nanoparticle, which can dramatically magnify its luminescence signal. In addition, the external silica layer protects the dyes from the surrounding matrix or other environment, which minimizes the photobleaching phenomena and increases its photostability. Moreover, silica possesses flexible routes for surface modifications with various types of functional groups (e.g. $-\mathrm{NH}_{2}$, $\mathrm{COOH},-\mathrm{SH}$ ), which allows its conjugation with different biomolecules during bioassays. To date, many promising NIRF dye-encapsulated silica nanomaterials have been reported with improved stability and detection sensitivity in biological applications. ${ }^{67}$ However, the main challenges of current applications comprise obstacles concerning the tedious operations, lack of sophisticated instrument and trained personnel in less-industrialized areas or in-field measurements. To address this issue, many attempts have been made to develop simple and lowcost point-of-care (POC) devices especially paper-based test strips. ${ }^{68}$ Compared to conventional test devices, paper test strips provide outstanding advantages including fast testing time, low cost, simple design and operation, which is attractive as a POC tool. ${ }^{12,69}$

In this study, we synthesized NIRF (cyanine 7 amine) dye encapsulated silica nanoparticles and integrated the nanomaterials as signal reporters into a lateral flow test strip. Compared with previous published methods for miRNA detection (summarized in Table 5.1), the present method can realize the direct detection of target miRNA in blood plasma samples without any sample pretreatment and complicated operation steps. It is because that the developed NIRF test strips take the advantages both from NIRF dyes and test strips, which avoids the issues of the 
interference problems from visual range, meanwhile holds the promises in POC and in-field applications. In addition, after loading the sample solution onto the NIRF test strips, the test results can be read out directly with a portable fluorescent reader within $40 \mathrm{~min}$. In the current study, circulating miRNA-34a was employed as the target molecule, owing to its potential in clinical applications as prognostic/predictive biomarkers in various forms of cancers, metabolic diseases and neurological diseases. ${ }^{70}$ Moreover, we also synthesized two VisFs encapsulated silica nanoparticles and compared their performances with that from the NIRF test strips. As a result, the NIRF test strips showed substantial improvement in detection sensitivity when target miRNAs were present in blood plasma specimens.

Table 5.1. Comparison of the present study with previous research from publications for miRNA detection.

\begin{tabular}{|c|c|c|c|c|c|}
\hline & Methods & Target miRNA & LOD & Linear Range & \\
\hline \multirow[t]{3}{*}{$\begin{array}{c}\text { Conventional } \\
\text { Method }\end{array}$} & $\begin{array}{l}\text { Northern } \\
\text { Blotting }\end{array}$ & $\begin{array}{l}\text { [1] miRNA-171 and } \\
\text { miRNA-319; }\end{array}$ & $\mathrm{pM}$ & N/A & $\operatorname{Ref}^{71}$ \\
\hline & QT-PCR & $\begin{array}{l}\text { [2] let-7a, miRNA-16, } \\
\text { miRNA-20, miRNA-21, } \\
\text { miRNA-22; } \\
\text { [3] miRNA-39, miRNA-54, } \\
\text { miRNA-238; } \\
\text { [4] hsa-miRNA-21, hsa- } \\
\text { miRNA-181b, hsa-miRNA- } \\
\text { 106a }\end{array}$ & nM-fM & $\mathrm{N} / \mathrm{A}$ & $\operatorname{Ref}^{72}$ \\
\hline & Microarray & $\begin{array}{c}\text { [5] chsa-miR-451 et al; [6] } \\
\text { Let-7d et al; [7] Let-7i }\end{array}$ & pM-fM & N/A & $\operatorname{Ref}^{73}$ \\
\hline \multirow[t]{2}{*}{ Method } & $\begin{array}{l}\text { Colorimetric } \\
\text { Method }\end{array}$ & $\begin{array}{c}\text { [8] miRNA-215; [9] } \\
\text { miRNA-224; [10] 0.1 pM }\end{array}$ & nM-fM & $\begin{array}{c}\text { [8] } 0.075 \text { to } 10 \\
\text { nM; [9] } 8 \mathrm{pM} \text { to } \\
75 \mathrm{nM} ;[10] 0.20 \\
\text { fM to } 10 \mathrm{pM}\end{array}$ & $\begin{array}{c}\operatorname{Ref}^{12} \\
38,74\end{array}$ \\
\hline & $\begin{array}{c}\text { Fluorescence } \\
\text { Method }\end{array}$ & $\begin{array}{l}\text { [11] let-7; [12] let-7; [13] } \\
\text { miRNA-21, miRNA-125b, }\end{array}$ & pM-aM & $\begin{array}{l}\text { [11] } 0.1 \mathrm{nM} \text { to } 0.1 \\
\mathrm{fM} \& 0.1 \mu \mathrm{M} \text { to }\end{array}$ & $\operatorname{Ref}^{75}$ \\
\hline
\end{tabular}




\begin{tabular}{|c|c|c|c|c|c|}
\hline & & miRNA-96 & & $\begin{array}{l}0.1 \mathrm{nM} ;[12] 50- \\
200 \mathrm{pM}[13] \mathrm{N} / \mathrm{A}\end{array}$ & \\
\hline & SPR & $\begin{array}{c}\text { [14] miR-21; [15] miRNA- } \\
21\end{array}$ & $\mathrm{pM}$ & $\begin{array}{c}\text { [14] } 10 \mathrm{pM}-\mu \mathrm{M} \\
\text { [15] } 5 \mathrm{pM} \text { to } \\
100 \mathrm{nM}\end{array}$ & $\operatorname{Ref}^{76}$ \\
\hline \multirow[t]{2}{*}{$\begin{array}{c}\text { Electrochemic } \\
\text { al Method }\end{array}$} & $\begin{array}{c}\text { Amperometric } \\
\text { Method }\end{array}$ & $\begin{array}{c}\text { [16] let-7a; [17] miRNA-21; } \\
\text { [18] miR-21 }\end{array}$ & fM-aM & $\begin{array}{c}\text { [16] } 10 \mathrm{fM}-1 \\
\mathrm{nM} ;[17] 0.1 \mathrm{pM}- \\
1 \mathrm{nM}[18] 10 \mathrm{fM} \\
\text { to } 10 \mathrm{nM}\end{array}$ & $\operatorname{Ref}^{77}$ \\
\hline & Impedimetric & $\begin{array}{c}\text { [19] miRNA-34a; [20] let- } \\
7 \mathrm{~b}\end{array}$ & $\begin{array}{c}{[19]} \\
28.1 \mathrm{pM} \\
{[20] \mathrm{fM}}\end{array}$ & $\begin{array}{c}\text { [19] } 0 \text { to } \sim 147 \\
\mathrm{pM} ;[20] 2.0 \mathrm{fM} \\
\text { and } 2.0 \mathrm{pM}\end{array}$ & $\operatorname{Ref}^{78}$ \\
\hline $\begin{array}{l}\text { Present } \\
\text { method }\end{array}$ & $\begin{array}{l}\text { Fluorescent } \\
\text { Method }\end{array}$ & MiRNA-34a & $\begin{array}{c}50 \mathrm{pM} \text { in } \\
50 \% \text { of } \\
\text { blood } \\
\text { plasma }\end{array}$ & $100 \mathrm{nM}-0.1 \mathrm{nM}$ & \\
\hline
\end{tabular}

\subsection{MATERIAL AND INSTRUMENTATIONS}

N-hydroxysuccinimide (NHS), 1-ethyl-3-(3-dimethylaminopropyl) carbodiimide (EDC) and tetraethyl orthosilicate (TEOS) were purchased from Sigma-Aldrich; $\mathrm{HAuCl}_{4}, \mathrm{Na}_{3} \mathrm{PO}_{4} \cdot 12 \mathrm{H}_{2} \mathrm{O}$, Tween 20, Triton X-100, trisodium citrate, PBS ( $\mathrm{pH}=7.4), \mathrm{Na}_{3} \mathrm{PO}_{4} \cdot 12 \mathrm{H}_{2} \mathrm{O}$, Bovine serum albumin (BSA), sodium chloride, streptavidin were purchased from Sigma-Aldrich and used without further purification. Sucrose was purchased from VWR. 3-Triethoxylsilylpropyl succinic anhydride (TEPSA) was obtained from Gelest Inc.

Silica nanosphere $(60 \mathrm{~nm})$ was purchased from Nanocomposix, Inc. Cyanine 7 amine (NIRF) was purchased from Lumiprobe, Alexa Fluor ${ }^{\mathrm{TM}} 633$ (VisF-1) was purchased from Life technologies, Rhodamine 6G (VisF-2) was purchased from Fluka. Blood plasma was purchased from US Biological Life Science (P4252-56 Plasma, Human, IgG Free).

Target miRNAs were purchased from Integrated DNA Technologies, Inc. (Coralville, IA) and have the following sequences:

Target miRNA-34a: 5'-UGG CAG UGU CUU AGC UGG UUG U -3' 
Detection probe (probe 1): 5'-/ $\mathrm{NH}_{2} /-\mathrm{AAA} A C A$ ACC AGC-3'

Capture probe (probe 2): 5'-ACA CTG CCA-/Biotin/-3'

Control probe (secondary probe): 5'-/Biotin/-GCT GGT TGT TTT AAA/-3'

Glass fibers (GFCP000800), cellulose fiber sample pads (CFSP001700), laminated cards (HF000MC100), and nitrocellulose membranes (HFB18004 and HFB 24004) were purchased from Millipore (Billerica, MA).

E/D cPOC fluorescent readers: (365 nm/615 nm; $632 \mathrm{~nm} / 650 \mathrm{~nm} ; 750 \mathrm{~nm} / 755 \mathrm{~nm})$ were purchased from LRE Medical GmbH.

\subsection{PREPARATION PROCEDURES}

\subsubsection{Preparation of Dye Encapsulated Silica Nanoparticles}
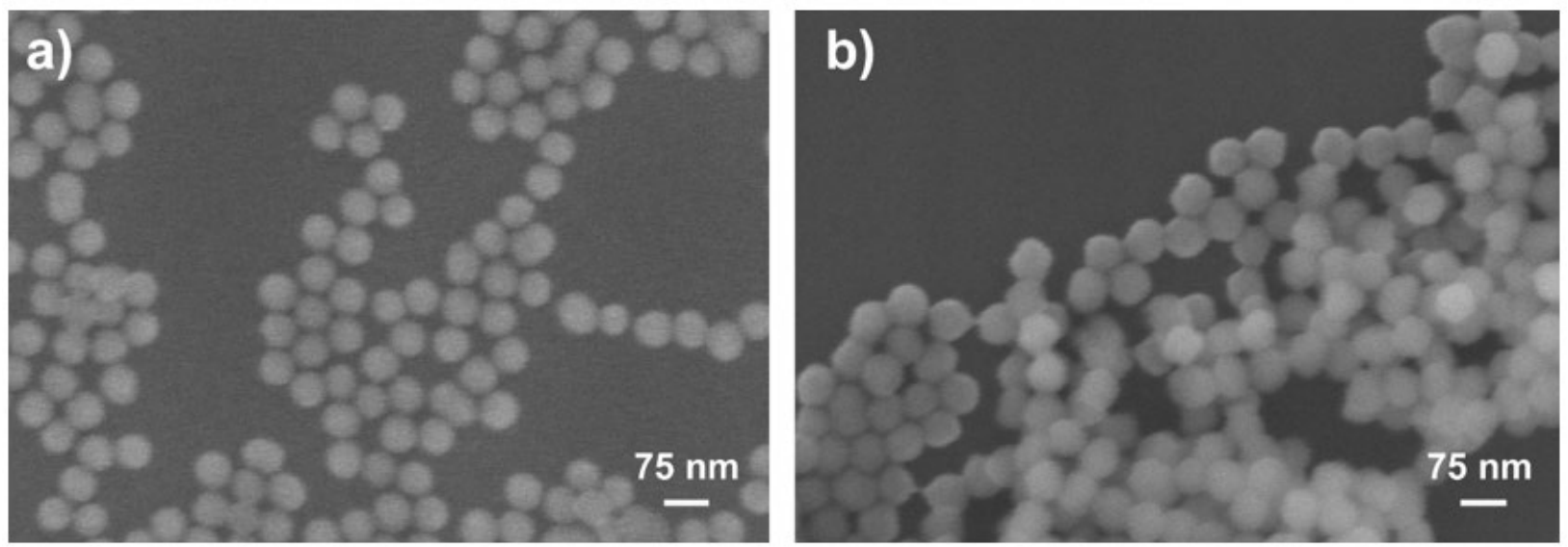

Figure 5.1. SEM images of a) commercial silica beads and b) synthesized NIRF nanoparticles.

The encapsulation of dyes was accomplished by coating of commercial silica nanospheres with an outer layer containing fluorescent dyes. In brief, $50 \mu \mathrm{L}$ of $100 \mu \mathrm{g} / \mathrm{mL}$ of fluorescent dye was mixed with $50 \mu \mathrm{g} / \mathrm{mL}$ of commercial silica nanospheres in $10 \mathrm{~mL}$ of ethanol solution. The obtained mixture was kept stirring for $20 \mathrm{~min}$. Then $10 \mu \mathrm{L}$ of TEOS and $10 \mathrm{uL}$ of $28 \%$ ammonia hydroxide were added into the solution with 20 min intervals. All of the above reactions were carried out under darkness. After overnight incubation, the reaction between TEOS and ammonia hydroxide leads to the formation of a silica shell on the dye-nanoparticle surface. The resulting nanoparticles were collected by washing and re-dispersion steps through centrifuging in ethanol solution for several times. The final nanoparticles were re-suspended in $1 \mathrm{~mL}$ of ethanol solution 
for future use. This encapsulation method guarantee the uniformity of the dye encapsulated silica nanoparticles and has potential to permit the synthesis of dye encapsulated silica nanoparticles with precisely tunable sizes through adjusting the amount of TEOS and ammonia hydroxide. Figure 5.1a and $\mathbf{b}$ show the SEM images of the commercial silica beads and the synthesized NIRF encapsulated silica nanoparticles respectively. From Figure 5.1b we can see that the NIRF nanoparticles were well dispersed and showing spherical morphology, which demonstrated that the silica outer layer was uniformly coated onto the silica core. Particle sizes were determined by Nano Measurer. The average particle sizes of the commercial silica beads and the NIRF nanoparticles were found to be $59.7 \pm 2.0 \mathrm{~nm}$ and $62.5 \pm 2.6$ respectively. The increase in size reveals that the thickness of the silica outer layer was at $\sim 5 \mathrm{~nm}$.

\subsubsection{Labeling Detection DNA with Nanoparticles}

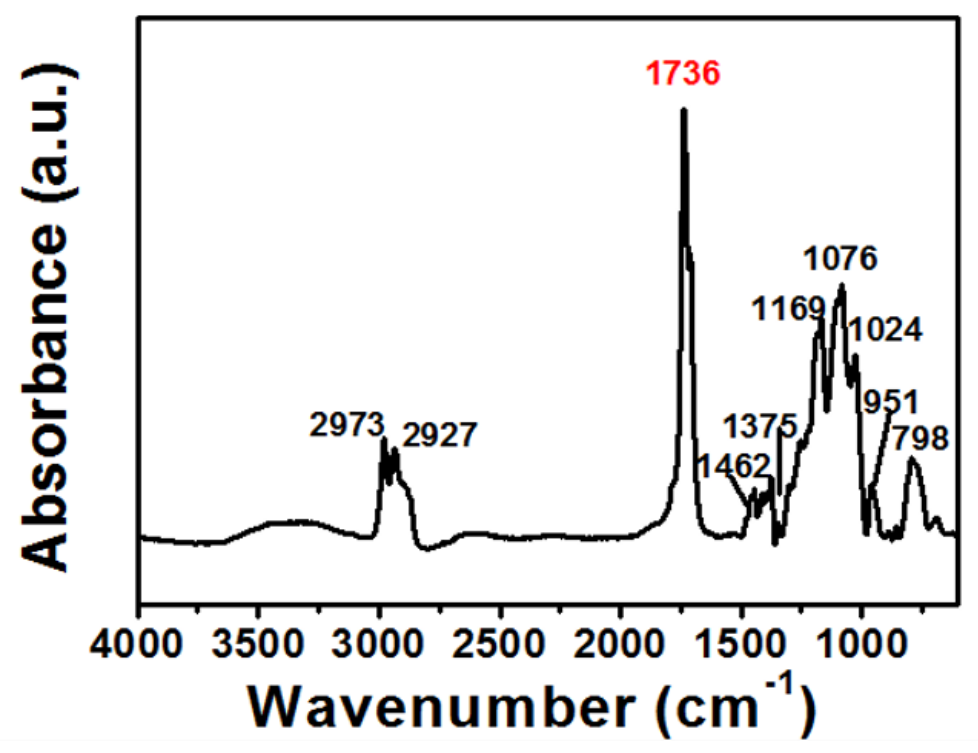

\begin{tabular}{|c|c|}
\hline $\begin{array}{c}\text { Peak Position } \\
\left(\mathrm{cm}^{-1}\right)\end{array}$ & Assignment \\
\hline 2973 & C-H stretch \\
\hline 2927 & C-H stretch \\
\hline 1736 & C=O stretch \\
\hline 1462 & C-H scissoring \\
\hline
\end{tabular}

Figure 5.2, FT-IR spectra of the carboxyl-group functionalized silica nanoparticles. The peak at $1736 \mathrm{~cm}^{-1}$ peak is assigned to the $\mathrm{C}=\mathrm{O}$ stretching vibration in $\mathrm{COOH}$ of TEPSA. 
The preparation of labeling DNA- $\mathrm{NH}_{2}$ with NIRF encapsulated nanoparticles followed the previously reported method. ${ }^{79}$ The obtained $1 \mathrm{~mL}$ of silica coated NIRF nanoparticles were mixed with $20 \mu \mathrm{L}$ of TEPSA. The mixture solution was incubated overnight to achieve carboxyl group-terminated NIRF nanoparticles. The immobilization of carboxyl group onto the surface of silica was verified through FTIR spectra (Figure 5.2), in which the peak at $1736 \mathrm{~cm}^{-1}$ indicated its successful linking. After washing with ethanol and D.I. water for several times, the resulting nanoparticles were re-suspended in $0.5 \mathrm{~mL}$ of the solution containing $50 \mathrm{mM}$ NHS and $200 \mathrm{mM}$ EDC. After incubation for $2 \mathrm{~h}$, the $\mathrm{COOH}$ group was activated. $50 \mu \mathrm{L}$ of $20 \mu \mathrm{M}$ ssDNA (amine group labeled detection DNA) solution was then added into the mixture. After overnight incubation, the solution was washed with a buffer solution (PBS containing $0.1 \%$ of BSA) for three times. The resulting nanoparticles were finally suspended in $200 \mu \mathrm{L}$ of Eluent buffer (20 $\mathrm{nM}$ of $\mathrm{Na}_{3} \mathrm{PO}_{4} \cdot 12 \mathrm{H}_{2} \mathrm{O}$ containing $5 \%$ BSA, $0.25 \%$ Tween 20 , and $10 \%$ sucrose) and stored under $4{ }^{\circ} \mathrm{C}$ for future use. The schematic representation of the synthesis procedure of the NIRF nanoparticle is shown in Figure 5.3. The successful labeling of amine group linked detection DNA onto silica was examined and proved by FTIR spectra. As shown in Figure 5.4, the peaks at $1655 \mathrm{~cm}^{-1}$ and $1550 \mathrm{~cm}^{-1}$ were assigned to the N-H bending from the amine group linked detection DNA.

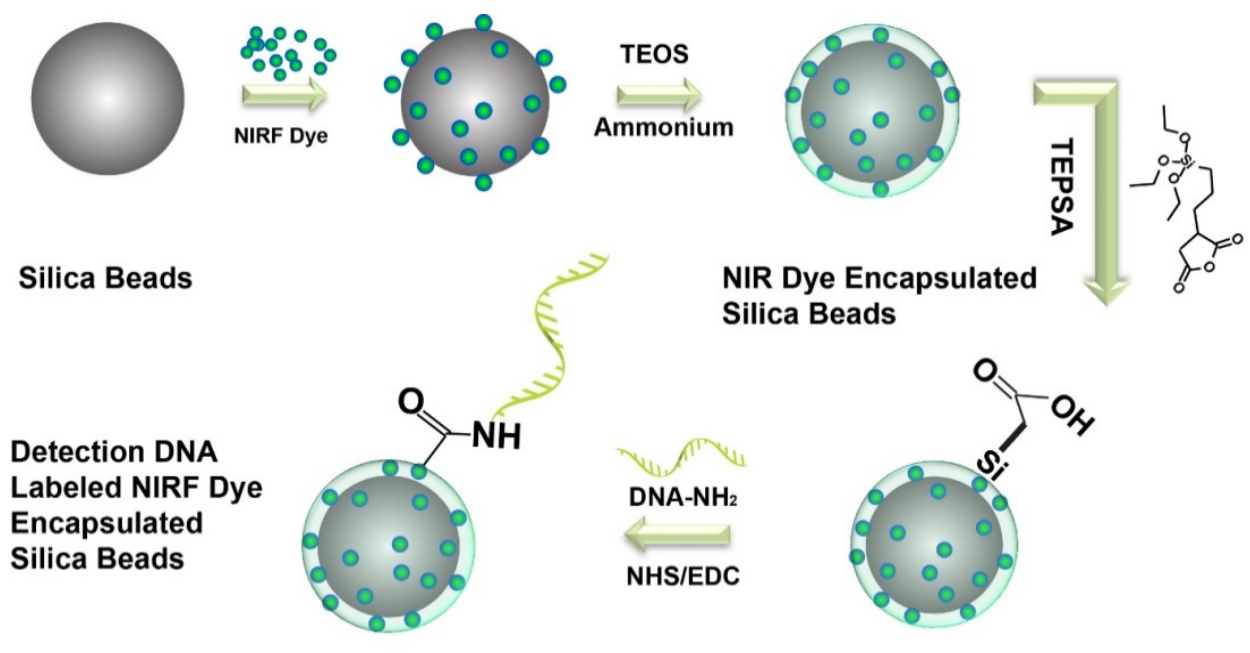

Figure 5.3. Schematic illustrations of NIRF encapsulated silica nanoparticles synthesis and labeling detection DNA probe with the NIRF encapsulated silica nanoparticles. 


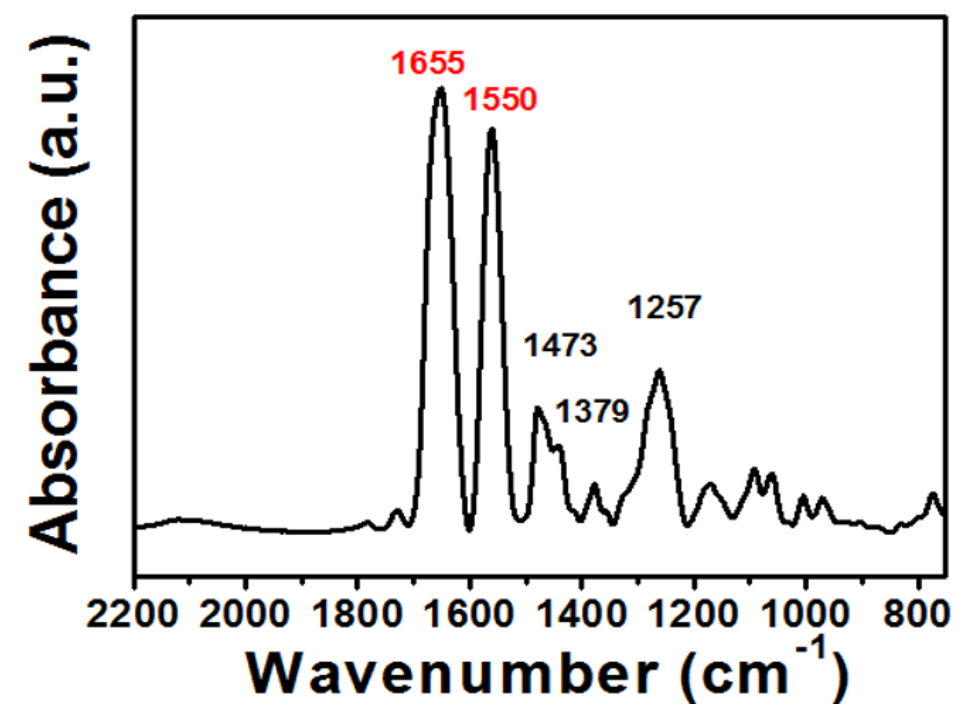

\begin{tabular}{|c|c|}
\hline $\begin{array}{c}\text { Peak Position } \\
\left(\mathrm{cm}^{-1}\right)\end{array}$ & Assignment \\
\hline 1655 & N-H bend \\
\hline 1550 & N-H bend \\
\hline
\end{tabular}

Figure 5.4, FT-IR spectra of the $\mathrm{NH}_{2}$ functionalized silica nanoparticles. The peak at $1655 \mathrm{~cm}^{-1}$ and $1550 \mathrm{~cm}^{-1}$ were assigned to the N-H bending from the amine group linked detection DNA.

\subsubsection{Preparation of Streptavidin-Biotinylated Capture DNA Conjugates}

The preparation of streptavidin-biotinylated DNA conjugates followed the previously reported methods. $200 \mu \mathrm{L}$ of $2.5 \mathrm{mg} / \mathrm{ml}$ of streptavidin was mixed with $50 \mathrm{mM}$ of biotinylated DNA probes (capture probe/control probe). The mixture was incubated on a shaker for $1 \mathrm{~h}$. After adding $500 \mu \mathrm{L}$ PBS into the mixture, the solution was centrifuged in dialysis tube for 20 minutes at $6000 \mathrm{rpm}$ under $4^{\circ} \mathrm{C}$. The above steps were repeated for 3 times to remove the unbound DNA. The remaining solution in filter was diluted to $600 \mu \mathrm{L}$ with PBS.

\subsubsection{Preparation of Test Strip}

The test strip consisted of four components: sample application pad, conjugate pad, nitrocellulose (NC) membrane, and absorption pad. All components were made from paper materials and they were laminated into a sheet of plastic pad orderly. The sample pad was made from cellulose fiber (GFCP000800) and saturated with a Tris- $\mathrm{HCl}$ buffer ( $\mathrm{pH}$ 8.0) containing 
$0.23 \%$ of Triton $\mathrm{X}-100,0.05 \mathrm{M}$ of Tris- $\mathrm{HCl}$ and $0.15 \mathrm{M}$ of $\mathrm{NaCl}$. Then, the pad was dried at $37^{\circ} \mathrm{C}$ for $2 \mathrm{~h}$ and stored in desiccators at room temperature (RT). The test line and control line on the NC membrane (HFB18004 and HFB 24004) were prepared by dispensing streptavidinbiotinylated capture probe and streptavidin-biotinylated control probe solutions respectively with a home-made dispenser. The distance between the test and control lines was $3 \mathrm{~mm}$. The membrane was then dried at $37^{\circ} \mathrm{C}$ for $1 \mathrm{~h}$ and stored at $4^{\circ} \mathrm{C}$ in a dry state. The successful functionalization of streptavidin-biotinylated DNA on the test line was verified through the peaks at $1575 \mathrm{~cm}^{-1}$ and $1542 \mathrm{~cm}^{-1}$ of FT-IR spectra in Figure 5.5, which presents the N-H bending of the biotin group. Finally, the sample pad, conjugate pad, NC membrane, and absorption pad were assembled on a plastic adhesive backing $(60 \mathrm{~mm} \times 30 \mathrm{~cm})(\mathrm{HF} 000 \mathrm{MC} 100)$. Each part overlapped $2 \mathrm{~mm}$ to ensure that the solution could migrate through the test strip during the assay. Test strips with a 3-mm width were cut using a paper cutter. The detection DNA probe linked NIRF nanoparticles were dropped on the conjugate pad prior to the test. The formation of the NIRF test strip was exhibited in Figure 5.6a.

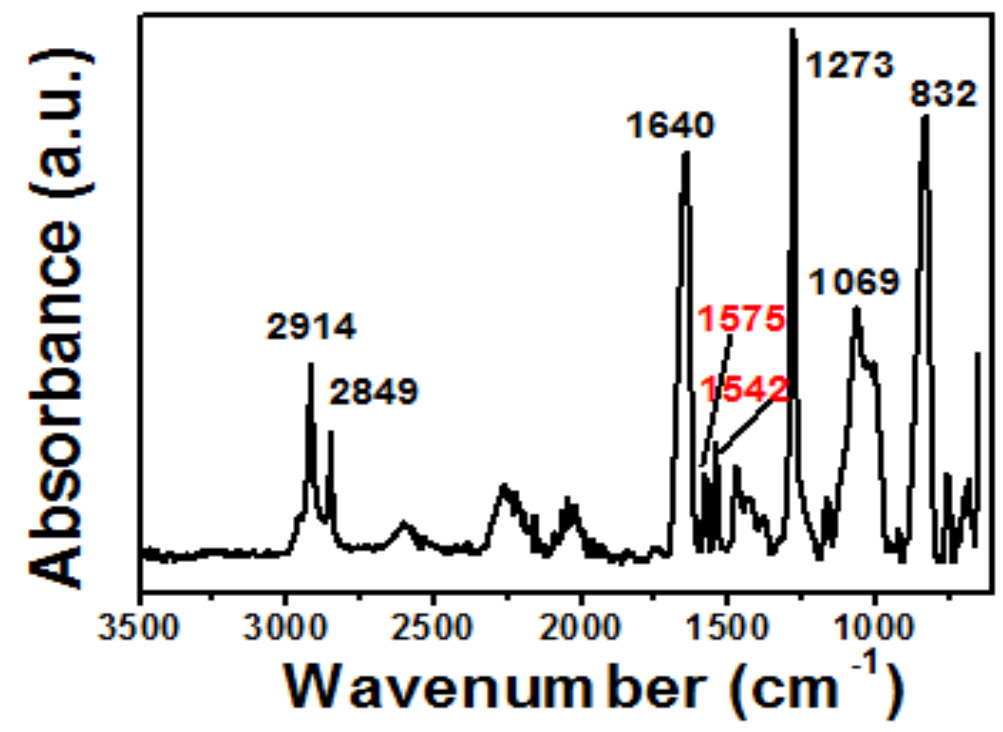

\begin{tabular}{|c|c|}
\hline $\begin{array}{c}\text { Peak Position } \\
\left(\mathrm{cm}^{-1}\right)\end{array}$ & Assignment \\
\hline 2914 & C-H stretch \\
\hline 2849 & C-H stretch \\
\hline 1640 & $\mathrm{NO}_{2}$ stretch \\
\hline
\end{tabular}




\begin{tabular}{|c|c|}
\hline 1575 & N-H bend \\
\hline 1542 & $\mathrm{~N}-\mathrm{H}$ bend \\
\hline 1273 & $\mathrm{NO}_{2}$ stretch \\
\hline 1069 & $\mathrm{C}-\mathrm{O}$ stretch \\
\hline 832 & $\mathrm{NO}_{2}$ stretch \\
\hline
\end{tabular}

Figure 5.5. FT-IR spectra of the capture DNA functionalized NC membrane. The peak at 1575 $\mathrm{cm}^{-1}$ and $1542 \mathrm{~cm}^{-1}$ were assigned to the N-H bending from the biotin group linked capture DNA probe.

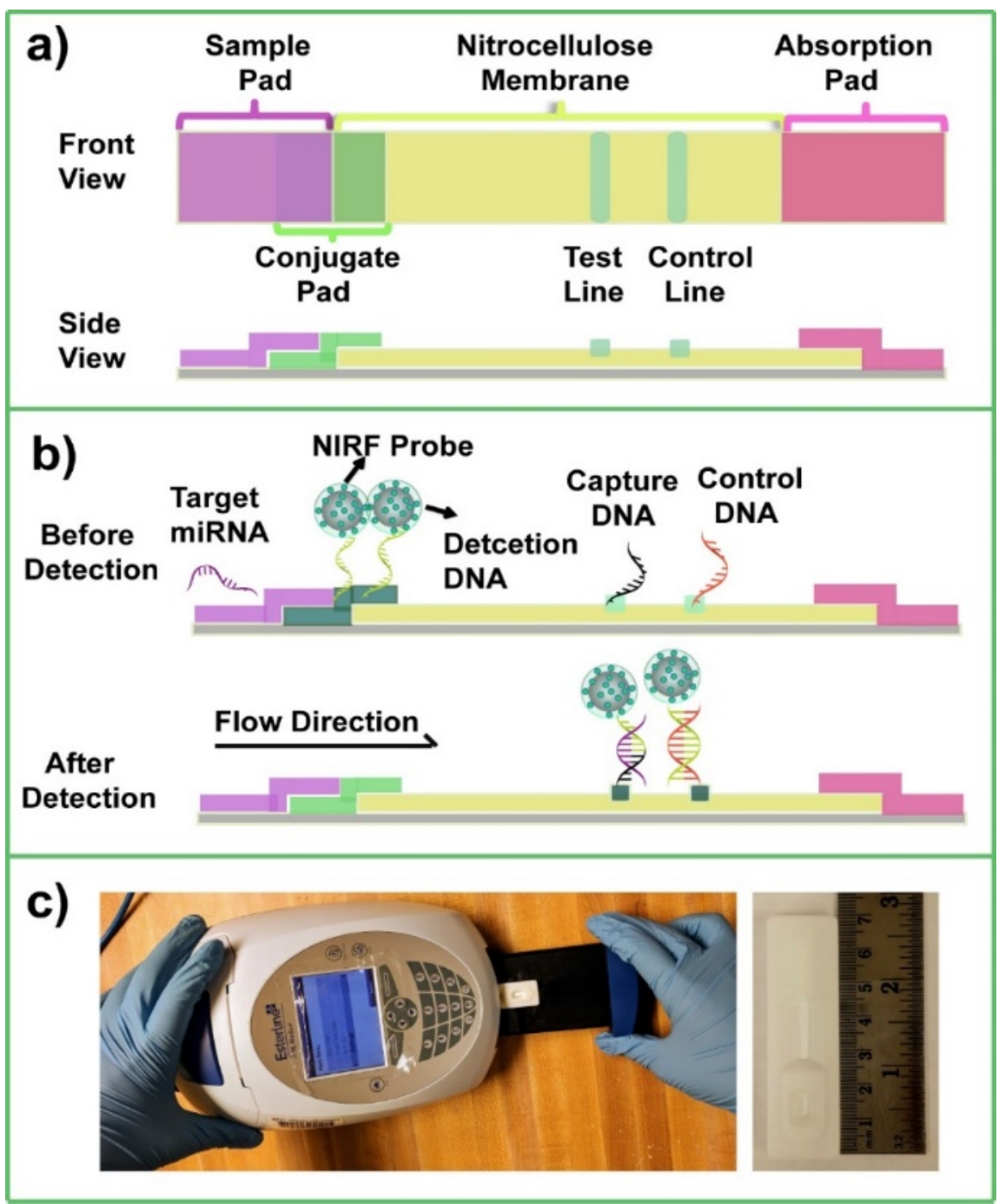

Figure 5.6. Schematic illustration of a) the formation of the paper-based NIRF test strip and b) 
miRNA-34 detection with the NIRF test strip; c) digital images of the portable fluorescent reader and NIRF test strip in cassette.

\subsection{ANALYTICAL CHARACTERIZATION}

\subsubsection{Operation Principle}

The operation principle of the NIRF-PLFS for target circulating miRNA detection is shown in Figure 5.6b. The overall mechanism of the detection process involves the reaction of (a) the formation of NIRF linked detection DNA-miRNA complex on the conjugate pad; (b) the complex from step (a) was captured on to the test line via the formation of a sandwiched structure of NIRF/detection DNA-miRNA-capture DNA; (c) the formation of a complex of NIRF/detection DNA-secondary DNA on control line. The schematic illustration of the above mentioned steps are respectively shown in Figure 5.7. Since the presence of target miRNA induces the formation of a fluorescent dye linked sandwich-structured complex onto the NIRF test strip, consequently, the fluorescent signal is directly proportional to the quantity of target miRNA. During a typical assay, a NIRF test strip was firstly loaded into a cassette (Figure 5.6c), then $100 \mu \mathrm{L}$ of sample solution was dropped into the sample window of the cassette. After that, owing to capillary force, the solution migrated along with the test strip from sample pad to conjugate pad, where the reaction between miRNA with detection DNA probe linked NIRF nanoparticles occurred. Then the resulted complexes kept moving along with the test strip until they reached the test line and captured by capture DNA through the formation of a sandwich structure (NIRF/detection DNA-miRNA-capture DNA). The rest of NIRF/detection DNA conjugates migrated over the test line and captured by the control line where the secondary DNA probe was immobilized. After reaction, the test strip was analyzed by a cPoc fluorescent strip reader, which is a portable handheld device (Figure 5.6c). During the data acquiring, the fluorescent peak intensity was used for quantitative work since the peak intensity is in proportion to the concentration of target miRNA. 


\section{Reaction on test line}

\section{Detection DNA Probe 3'-CG ACC AAC A AAA-/ $\mathrm{NH}_{2} /-5$ ' \\ 5'-UGG CAG UGU CUU AGC' ÚG'G UUUG Ú-3' Target miRNA I I I II I I}

3'-/Biotin/-ACC GTC ACA-5' Capture DNA Probe

\section{Reaction on control line}

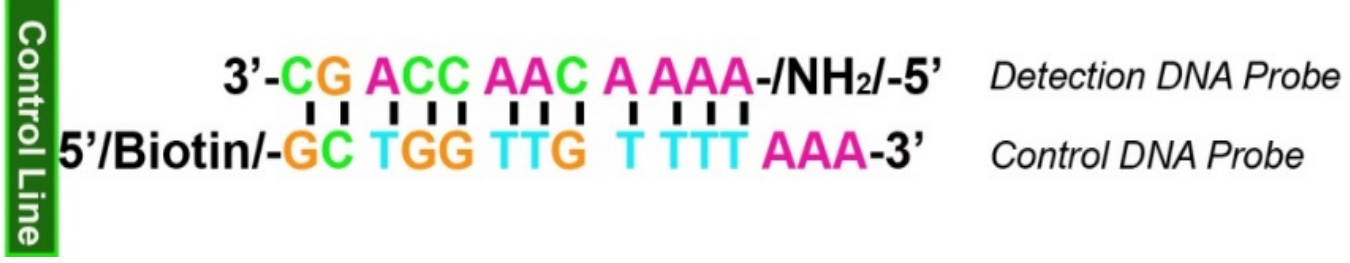

Figure 5.7. Formations of complex of (NIRF/detection DNA-miRNA-capture DNA) and (NIRF/detection DNA-control DNA) on test line and control line respectively.

\subsubsection{Parameter Optimizations}

The running buffer is a critical experimental component affecting the signal readout, which plays a crucial role in increasing detection signal and reducing nonspecific adsorption of fluorescent probes on test line. In the current study, TWEEN 20 was used as surfactant in running buffer to control the flow rate and reduce the background fluorescent signal. As shown in Figure 5.8a, with the increasing amount of TWEEN 20, the fluorescent $\mathrm{S} / \mathrm{N}$ ratio rises gradually and then tends to a constant value at $0.3 \%$, which is chosen as the optimized TWEEN 20 amount. The reaction time is another important parameter of the NIRF test strip, which improves the bounding stability of detection reagent and target miRNA with the capture DNA immobilized test line. Meanwhile, increasing reaction time can help reduce background signal as the nonspecific adsorbed fluorescent probe on test line can be washed away by the running buffer. The reaction time was optimized from 1 to $50 \mathrm{~min}$, and as shown in Figure $\mathbf{5 . 8 b}$, the optimal $\mathrm{S} / \mathrm{N}$ is obtained from the reaction at $40 \mathrm{~min}$. 

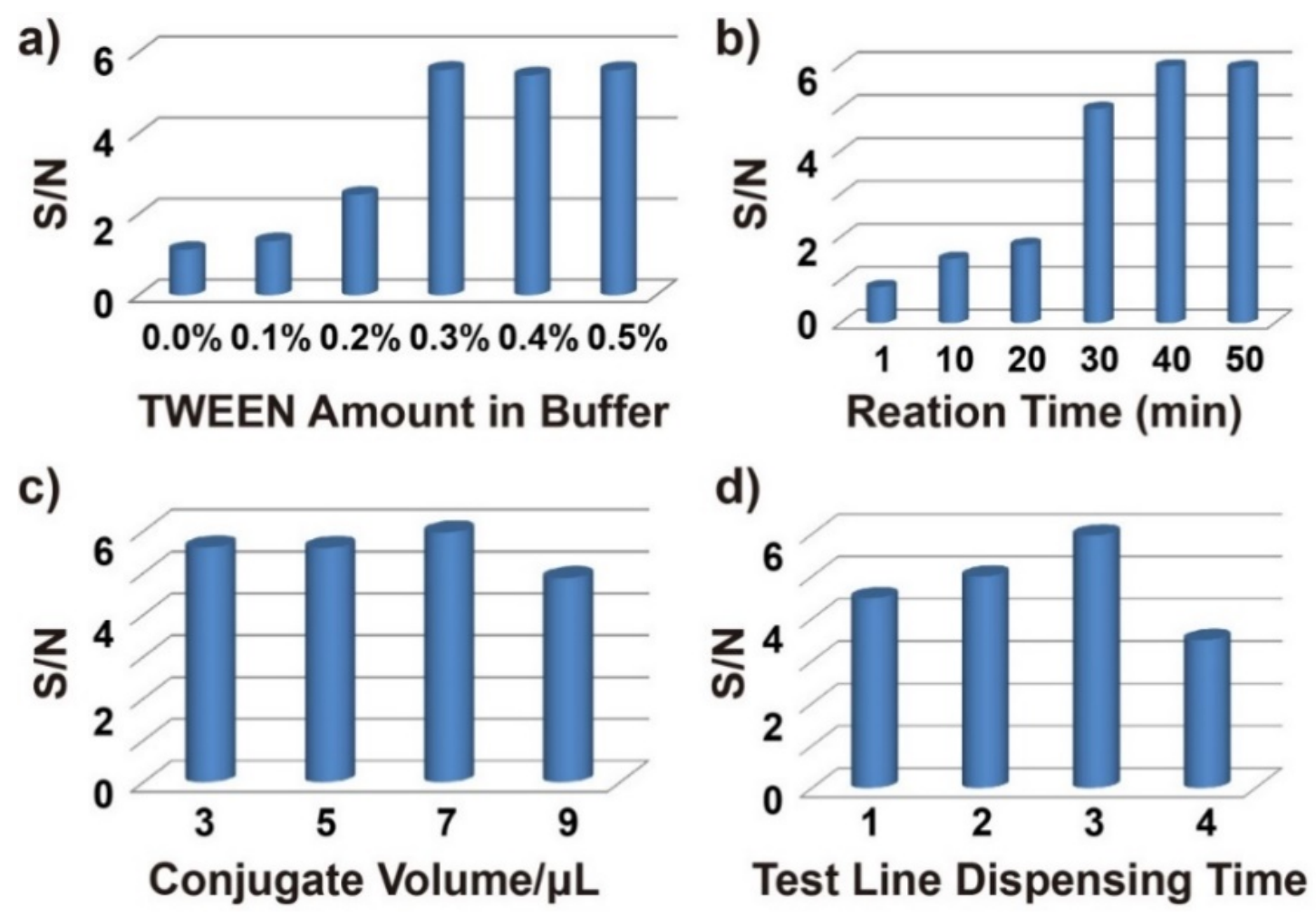

Figure 5.8. Experiment optimizations with NIRF test strips for miRNA measurements. a) Effect of surfactant of TWEEN 20 amount in running buffer; B) effect of the reaction time; C) effect of the volume of NIRF/detection DNA conjugate; D) effect of the dispensing times of the capture DNA on test line on the $\mathrm{S} / \mathrm{N}$ ratio of the NIRF based test strip.

The amounts of detection DNA-NIRF conjugate and capture DNA on test line can substantially affect the signal intensity. From Figure 5.8c we can see that with the conjugate volume increase, the $\mathrm{S} / \mathrm{N}$ ratio increases because more NIRF nanoparticles are captured by test line in the present of miRNA. When the volume of conjugate exceeds $7 \mu \mathrm{L}$, the $\mathrm{S} / \mathrm{N}$ ratio decreases owing to that the increased non-specific adsorption of NIRF nanoparticles on test line. Therefore, $7 \mu \mathrm{L}$ of NIRF conjugate is optimal. The capturing capacity of NIRF nanoparticles onto test line is depended on the capturing reagent amount. In the current study, 3 times of dispensing of capturing reagent onto test line was found to be the optimal and used in the following experiment (Figure 5.8d).

\subsubsection{Stability Comparison}



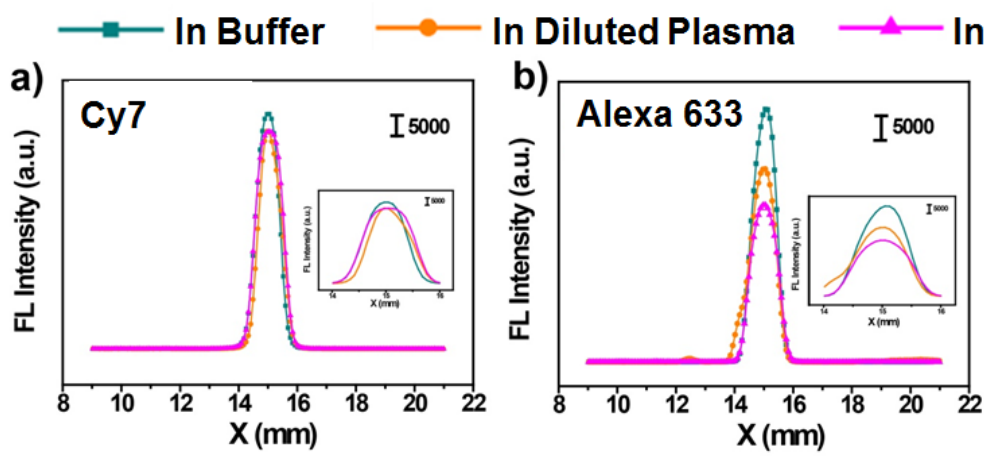

In Plasma

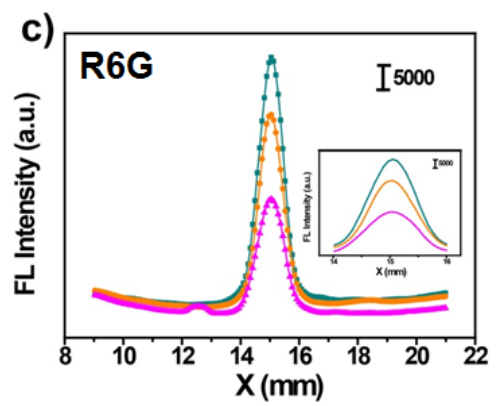

Figure 5.9. Stability comparison of different dyes: a) Cy7, b) Alexa 633 and c) Rhodamine 6G encapsulated silica nanoparticles in buffer, $50 \%$ of plasma and $100 \%$ of plasma.

In order to demonstrate the advantages of NIRF in increasing the photostability and antiinterference capacity, the effect of biological matrices on the fluorescent intensity of the NIRF and VisF dyes encapsulated silica nanoparticles was investigated. The results are exhibited in Figure 5.9. The $X$ axis of the figures presents the position of the nanoparticles on the test strip. Figure 5.9a, b and $\mathbf{c}$ respectively describes the fluorescent spectra of NIRF (Cy7), VisF-1 (Alexa 633) and VisF-2 (Rhodamine 6G) encapsulated silica nanoparticles in the present of buffer (green line with squares), 50\% of blood plasma (orange line with spheres) and 100\% of blood plasma (pink line with triangles). The corresponding excitation/emission spectra of the employed dyes are shown in Figure 5.10.

a) NIRF-Cyanine 7 amine

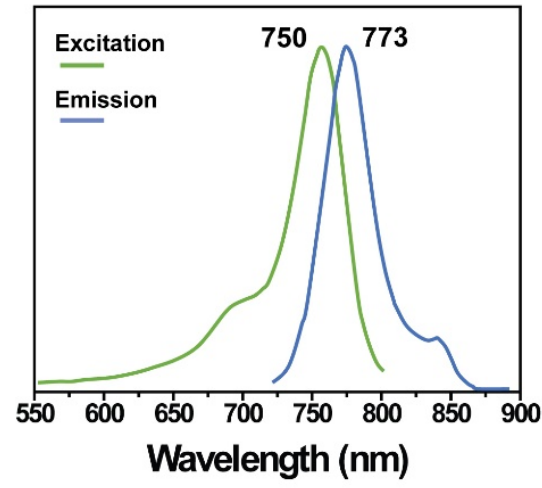

b) VisF-1-Alexa 633

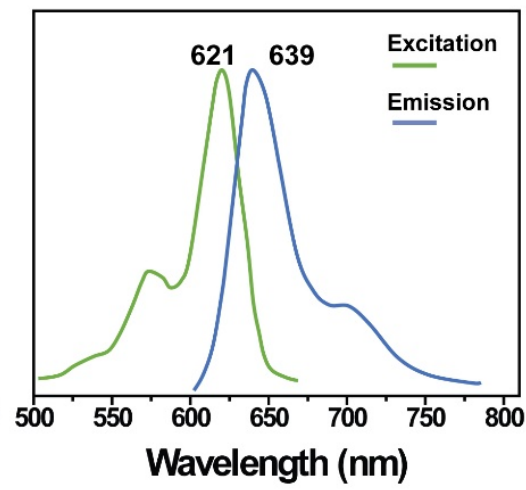

c) VisF-2-Rhodamine 6G

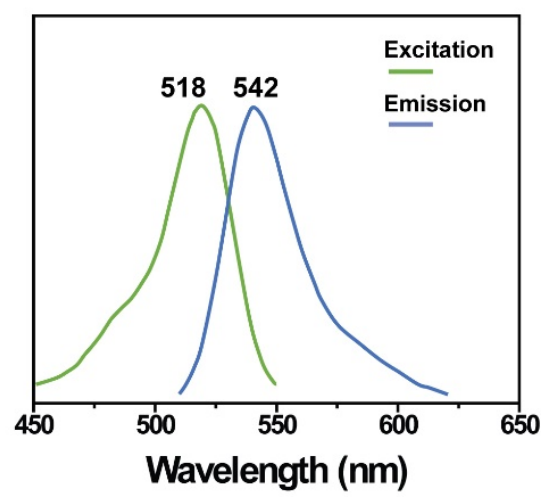

Figure 5.10. Excitation and emission spectra of the a) cyanine; b) Alexa 633 and c) Rhodamine $6 \mathrm{G}$ respectively. The information source of the spectra is from Lumiprobe, Life technologies and 
Fluka respectively.

From Figure 5.9a we can see that there is no manifest change in the fluorescent intensity when NIRF nanoparticles were suspended even in $100 \%$ of blood plasma specimen in comparison to that in running buffer. This is because that the wavelengths of the NIRF nanoparticles fall within the biological window, which provides increased transparency toward biological matrices. As a result, in that optical region, there is less absorbance, minimal scattering and reduced background fluorescent interference, which endowing the NIRF nanoparticles with favorable stability in biological matrices and, accordingly, offering accurate measurement for bioanalysis. In contrast to the NIRF nanoparticles, the VisF-1 encapsulated silica nanoparticles showed fluorescent intensity decreases by $23.6 \%$ and $37.8 \%$ (Figure 5.9b) respectively in $50 \%$ and $100 \%$ of blood plasma. The results implied that there was great scattering interference from biological matrix, which severely influence its luminance intensity. In the case of VisF-2, the situation was even worse. Substantial signal decreases were observed from the VisF-2 nanoparticles when they existed in the biological matrices (Figure 5.9c). Moreover, the baselines of the fluorescent spectra were wavy and oscillating, which were contributed to the background interference from test strip materials and surrounding environment, leading to imprecise signal response and low detection sensitivity. Interestingly, compared with VisF-2 nanoparticles (Figure 5.9c), VisF-1 nanoparticles (Figure 5.9b) possessed smooth and flat spectrum baselines, indicating that the background interference played less effect on the VisF-2 nanoparticles. This is because that the wavelengths of VisF-2 nanoparticles fall near the left border of the NIR region, which endowed the nanoparticles with improved anti-interference capacity towards the biological matrices and surrounding environment. The above findings revealed that the NIRF played a vital role in elevating the photostability and avoiding the background interference from surrounding environment, holding promises in direct bioanalysis.

\subsubsection{Analytical Characterization}

In order to evaluate the performances of the NIRF-PLFSs, sample solutions containing different concentrations of miRNA-34 (ranging from 0 to $100 \mathrm{nM}$ ) were measured under the optimal experimental conditions. As descripted in Figure 5.11a, the fluorescent response ((I$\left.\mathrm{I}_{0}\right) / \mathrm{I}_{0}$ ) linearly increases with the increasing of logarithm of target circulating miRNA concentration, and the corresponding regression equation was $y=7.2 x+18.6$ with a correlation coefficient of 0.93 . The detection limit of the NIRF-PLFS for target circulating miRNA-34 was 
found to be $20 \mathrm{pM}$, which was estimated based on three times of signal-to-noise ratio. A linear detection range from $50 \mathrm{pM}$ to $100 \mathrm{nM}$ was obtained and was suitable for quantitative work. The above results implied that the developed NIRF-PLFS were working properly and reliably. The fluorescent signal stability was determined through measuring NIRF-PLFS with three concentration levels of miRNA-34 (50 nM, $1 \mathrm{nM}$ and $0 \mathrm{nM})$. Each measurement of miRNA was repeated six times on six NIRF-PLFS. Results for the three concentration levels of miRNA yielded a RSD respectively of $9.0 \%, 3.4 \%$ and $2.7 \%$. The results exhibited outstanding reproducibility, which clearly verified that the silica layer ameliorated the stability of the NIRF encapsulated nanoparticles, thereby allowing reproducible fluorescent responses to be obtained from different test strips constructed under the same condition.

In order to validate the anti-interference performance of the NIRF-PLFS in the present of complex biological samples, various concentrations of miRNA was spiked with $50 \%$ of blood plasma solutions. Then the resulted mixtures were examined by the NIRF-PLFSs. The test results were described in Figure 5.11b. Compared with the data from Figure 5.11a, under the same concentration of miRNA, there were no substantial signal decreases observed when the measurements were carried out in $50 \%$ of blood plasma, which indicated that the complex sample matrix exhibited very slight effect on the NIRF detection. As a result, a LOD of $50 \mathrm{pM}$ of miRNA was achieved when the NIRF-PLFSs were operated in $50 \%$ of blood plasma. A calibration curve was obtained by plotting the response versus logarithm of miRNA concentration, which was ranged from $0.1 \mathrm{nM}$ to $100 \mathrm{nM}$. 

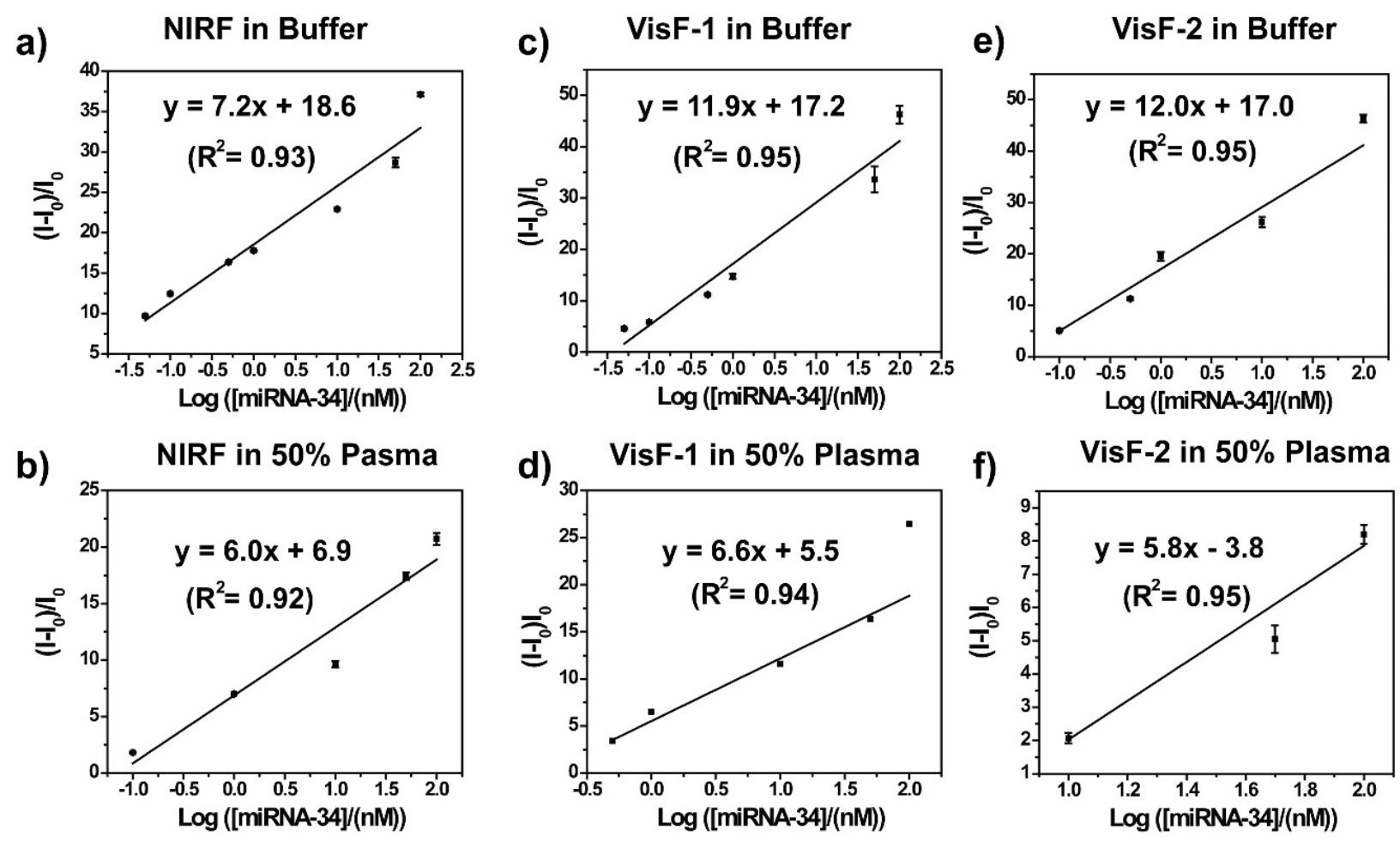

Figure 5.11. MiRNA-34 detection with various concentrations in buffer with a) NIRF, c) VisF-1 and e) VisF-2 nanoparticles incorporated test strips; and in 50\% of plasma with b) NIRF, d) VisF1 and f) VisF-2 nanoparticles incorporated test strips. (I presents the fluorescent intensity in the present of various concentrations of miRNA-34; while $\mathrm{I}_{0}$ presents the fluorescent intensity in the absent of miRNA-34).

\subsubsection{Performance Comparison}

\begin{tabular}{|c|c|c|c|c|}
\hline & & $\begin{array}{c}\text { NIRF test strip } \\
(\mathbf{C y 7})\end{array}$ & $\begin{array}{c}\text { VisF-1 test strip } \\
\text { (Alexa 633) }\end{array}$ & $\begin{array}{c}\text { VisF-2 test strip } \\
\text { (Rhodamine 6G) }\end{array}$ \\
\hline \multirow{2}{*}{ Buffer } & $\begin{array}{c}\text { Linear } \\
\text { Range }\end{array}$ & $100 \mathrm{nM}-50 \mathrm{pM}$ & $100 \mathrm{nM}-50 \mathrm{pM}$ & $100 \mathrm{nM}-0.1 \mathrm{nM}$ \\
\cline { 2 - 5 } & LOD & $20 \mathrm{pM}$ & $5 \mathrm{pM}$ & $50 \mathrm{pM}$ \\
\hline \multirow{5}{50\%}{} & Linear & $100 \mathrm{nM}-0.1 \mathrm{nM}$ & $100 \mathrm{nM}-0.5 \mathrm{nM}$ & $100 \mathrm{nM}-10 \mathrm{nM}$ \\
& Range & & & $5000 \mathrm{pM}$ \\
\cline { 2 - 5 } & LOD & $50 \mathrm{pM}$ & $100 \mathrm{pM}$ & \\
\hline
\end{tabular}


Table 5.2. Comparisons of test results from VisF and NIRF test strips for miRNA detection in buffer and in $50 \%$ of blood plasma samples.

To further verify the advanced anti-interference capacity of NIR nanoparticles, the comparisons among the VisF-1, VisF-2 and the NIRF incorporated PLFSs were conducted through detecting target miRNA in $50 \%$ of blood plasma specimens. In order to facilitate the comparison, all of the synthesis procedures and tests conditions of the NIRF and the VisFs test strips were kept same. The obtained calibration curves and the corresponding data including LODs and linear ranges were presented and summarized respectively in Figure 5.11 and Table 5.2. From the data we can see that in running buffer, the LODs for target miRNA from the VisF2 and NIRF-PLFSs were at the same concentration level. But when the measurements were carried out in 50\% of blood plasma, the LOD from NIRF-PLFS was 100 times lower than that from the VIF-2-PLFSs (5000 pM vs $50 \mathrm{pM}$ ). In addition, in comparison to the fluorescent responses from VisF-2-PLFSs for measuring $10 \mathrm{nM}$ of miRNA in running buffer, a five-time decrease of fluorescent response was observed when the tests were conducted in $50 \%$ of blood plasma samples (Figure 5.11b and f). The severe signal drops are attributed to substantial scattering loss of the excitation and emission light in the biological matrix. In contrast, the fluorescent responses from the NIRF-PLFSs were found to be very stable even for low concentration detection of target miRNA in $50 \%$ of blood plasma samples. Moreover, the slope of the calibration curve from the NIRF-PLFSs was comparable to that from the running buffer, which demonstrated that the detection sensitivity of the NIRF-PLFS wasn't affected significantly when the system was operated in biological samples. Consequently, the LOD for miRNA on the NIRF-PLFS was found to be $50 \mathrm{pM}$, which was at the same concentration level compared to that from the running buffer.

\subsection{CONCLUSION}

In this work, we developed a strategy for the synthesis of novel NIRF dye encapsulated silica nanoparticles through coating of commercial silica beads with NIRF dyes and a silica outer layer. The silica coating layer enables numerous NIRF to be encapsulated into the nanoparticle, leading to substantial fluorescent enhancement. Additionally, the silica layer can protect the dyes 
from the complex biological matrix. Moreover, the NIRF nanoparticles present advantages over VisF nanomaterials in terms of improved anti-interference ability and minimized background interference signal. As a result, the NIRF-PLFSs showed a two-order of magnitude improvement in detection sensitivity for miRNA in 50\% of blood plasma samples, which is expected to hold promise as a POC tool in biological assays and other bio-applications. 


\section{Chapter 6: AMPLIFICATION STRATEGY MODIFIED TEST STRIP IN PLASMA TESTING}

\subsection{NUCLEASE-ASSISTED AMPLIFICATION TECHNIQUE}

MicroRNAs (miRNAs) are a class of single-stranded non-coding RNA, which regulate gene expression by either inhibition of translation or mRNA degradation. ${ }^{80}$ Studies have shown that alterations in miRNA-expression play a significant effect on biological processes of disease development and progression, including proliferation, differentiation and apoptosis. ${ }^{81}$ Thus, miRNAs have become clinical promising biomarkers and have been explored in a variety of studies. Accurate and sensitive measurement of miRNAs holds great significance for detecting and predicting the resistance of different types of malignancies as well as for monitoring treatment response. ${ }^{82}$ However, the major challenge of miRNA measurement is that the low abundance of microRNAs but the high sequence homology within miRNA families in tissues, cells and circulating system. Hence the standard approaches for miRNA measurements, including Northern blotting and microarray are hindered by their intrinsic low sensitivity and specificity limitations. The quantitative reverse transcription-polymerase chain reaction (qRTPCR) is a well-established gold standard method for miRNA measurements, which exhibits dramatically improvement in detection sensitivity, leading to single molecule assay. ${ }^{83}$ However, the short length of miRNAs requires the design of short primers and precise control of temperature cycling for successful amplification, which decreases the efficiency of PCR and increases the chances of nonspecific amplification. Moreover, the approaches depend on laboratory-oriented sophisticated instruments, expert personnel and tedious operations, which severely hamper their practical application, especially in remote and resource-limited and areas.

Recently, a variety of novel amplification strategies have been developed to improve the detection sensitivity and adaptability for miRNA detection, including nanomaterial-based miRNA detection ${ }^{84}$ and various kinds of nucleic acid amplification techniques ${ }^{75 c, 85}$, including rolling circle amplification (RCA), ${ }^{86}$ exponential isothermal amplification (EXPAR) ${ }^{87}$ and nuclease-assisted amplifications (NAA). ${ }^{88}$ Nanomaterial-based amplification mainly depends on the unique properties of nanomaterials, such as inherent high carrier capacity, and outstanding conductivity and catalytic ability, offering the platform with eminent optical and electrochemical 
features. ${ }^{89}$ While NAA techniques primarily rely on an enzymatic process, which generates thousands to millions of copies of a specific DNA segment. For instance, the RCA technique enables the amplifications of a short DNA or RNA primer in the present of a circularized padlock probe and the corresponding special DNA or RNA polymerases. RCA is a powerful tool to detect DNA mutation, owing to the fact that even a single mismatch in the ligation junction does not allow circularizing of the probe (Rolling circle amplification: a versatile tool for chemical biology, materials science and medicine. ${ }^{90}$ In the case of EXPAR technique, one simple template and two enzymes are required. The two enzymes are respectively utilized in nicking endonuclease reaction and polymerase strand extension. EXPAR technique has gained increase attention in amplifying of low-abundance miRNA due to its high sensitivity and good tolerance to the inhibitory components in the clinical samples. ${ }^{91}$

Although the above mentioned techniques offer some advantages in miRNA amplification, they have practical limitations in time, cost, and simplicity. Among the NAA techniques, the duplex-specific nuclease (DSN) assisted signal amplification offers a rapid, convenient but powerful alternative strategy for highly sensitive miRNA detection. It enables the linear signal amplification without changing the quantity of the target miRNA. ${ }^{92}$ In addition, the DSNmediated signal amplification strategy owns unique advantages of high amplification efficiency, isothermal nature, and no nonspecific priming. The mechanism of DSN assisted amplification strategies are based on the cleavage of the single strand DNA probe only when it is in a DNARNA hybrid, while keeping the RNA intact. Then the target RNA acts as a template for a fresh DNA probe and repeats the process of hybridization and cleavage, leading to signal amplification. ${ }^{93}$ Various transducers have been integrated into DSN based sensors for achieving ultra-sensitivity for miRNA measurements, including colorimetry, ${ }^{93,}, 94$ fluorescence ${ }^{88 b, 95}$ and electrochemistry. ${ }^{96}$ Although the procedures have been simplified compared with other NAA techniques, most of the reported DSN based sensors still suffer from complicated nanofabrication and multiple step operations, which cannot meet the demands of point-of-care (POC) applications.

Paper-based lateral flow strips (PLFSs) are one of the best well-known POC tools, which possess the advantages of user friendly operation, portability and low cost. PLFSs have been employed in a variety of fields, such as environmental monitoring, food safety, and clinical diagnosis for detecting various biomolecules and chemical contaminants. However, PLFSs 
rarely involves in detection of early-stage diseases, owing to the fact that the sensitivity and accuracy of a PLFT are always sacrificed in order to simplify test procedures and accelerating sample screening. In the current study, in order to realize high sensitivity measurement of miRNA, the DSN assisted signal amplification strategy was introduced to modify PLFSs. The developed DNS-PLFSs can sustain the properties of a POC tool, at the same time, realizing ultrasensitive measurement of miRNA. In addition, near-infrared fluorescent NIRF (dylight 755) encapsulated silica nanoparticles were employed as signal reporters, which can further amplify signal response, meanwhile reduce the interference signal of the non-specific biomolecules from human fluids. As a result, the proposed device allowed a quantitative evaluation of miRNA-34a with a detection limit (LOD) of $0.1 \mathrm{pM}$ in running buffers and a LOD of $0.5 \mathrm{pM}$ in blood plasma samples. The obtained LOD of miRNA-34a is below the cut-off value of that in the plasma samples of stroke patients, exhibiting great potential as a POC miRNA quantification tool for use in biomedical research and clinical diagnosis. Moreover, compared with other miRNA amplification methods, the developed DSN-PLFS shows advantages in terms of simplicity, low cost, fast response (within $40 \mathrm{~min}$ ) and user-friendly properties.

\subsection{MATERIALS AND METHODS}

\subsubsection{Reagents and Materials}

N-hydroxysuccinimide (NHS), 1-ethyl-3-(3-dimethylaminopropyl) carbodiimide (EDC) and tetraethyl orthosilicate (TEOS) were purchased from Sigma-Aldrich; $\mathrm{HAuCl}_{4}, \mathrm{Na}_{3} \mathrm{PO}_{4} \cdot 12 \mathrm{H}_{2} \mathrm{O}$, Tween 20, Triton X-100, trisodium citrate, PBS ( $\mathrm{pH}=7.4)$, Bovine serum albumin (BSA), sodium chloride, streptavidin were purchased from Sigma-Aldrich and used without further purification. Sucrose was purchased from VWR. 3-Triethoxylsilylpropyl succinic anhydride (TEPSA) was obtained from Gelest Inc. Silica nanosphere $(60 \mathrm{~nm})$ was purchased from Nanocomposix, Inc. DyLight ${ }^{\mathrm{TM}} 755$ NHS Ester was purchased from Thermo Scientific. Blood plasma was purchased from US Biological Life Science (P4252-56 Plasma, Human, IgG Free).

Duplex-specific nuclease (DSN) kit (containing DSN enzyme, DSN storage buffer, 10X DSN master buffer, 2X DSN stop solution and control template) was purchased from Evrogen. RNase inhibitor was purchased from Sigma-Aldrich. Water glass distilled PCR Certified was purchased from VWR.

Target miRNAs, detection probes and capture probes were purchased from Integrated DNA 
Technologies, Inc. (Coralville, IA) and have the following sequences:

Target miRNA-34a:

5'-UGG CAG UGU CUU AGC UGG UUG U -3'

miRNA sequence with two mismatches:

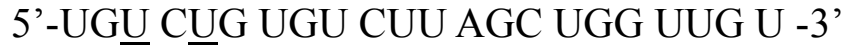

Detection probe:

5'-/AmMC6/-GCG CGC GCA CAA CCA GCT AAG ACA CTG CCA-/Bio/-3'

Capture probe:

5'-/Biotin/GCG CGC GC-3'

Glass fibers (GFCP000800), cellulose fiber sample pads (CFSP001700), laminated cards (HF000MC100), and nitrocellulose membranes (HFB18004 and HFB 24004) were purchased from Millipore (Billerica, MA). E/D cPOC fluorescent readers $(750 \mathrm{~nm} / 755 \mathrm{~nm})$ was purchased from LRE Medical GmbH.

\subsubsection{Instruments and Characterization}

Fourier transform infrared (FT-IR) spectra were obtained with Thermo Nicolet 6700 spectrometer. A scanning electron microscope (JEOL-JSM-7600F) was used to image the fluorescent dye encapsulated nanoparticles.
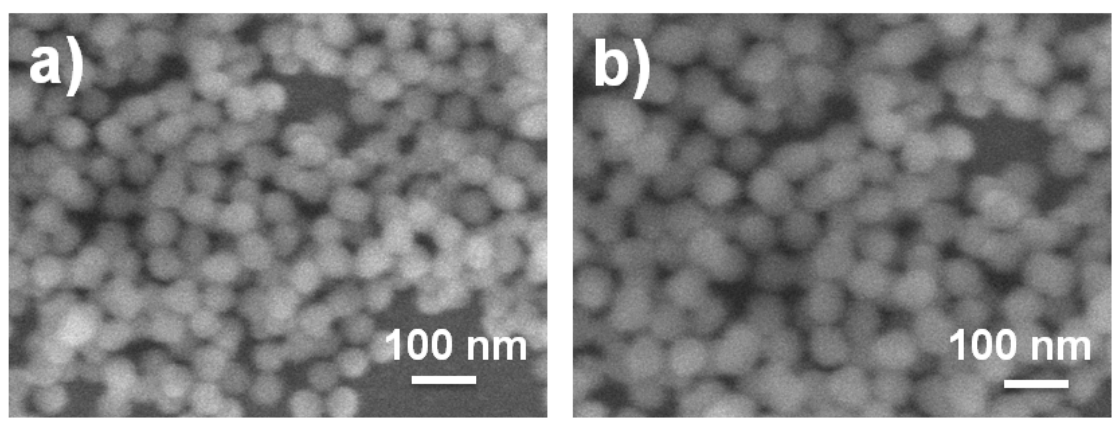

Figure 6.1. SEM images of a) commercial silica beads and b) synthesized NIRF nanoparticles.

\subsubsection{Synthesis of Nanoparticles}

The encapsulation of dyes was accomplished by coating of commercial silica nanospheres with an outer layer containing fluorescent dyes. In brief, $50 \mu \mathrm{L}$ of $100 \mu \mathrm{g} / \mathrm{mL}$ of fluorescent dye was mixed with $50 \mu \mathrm{g} / \mathrm{mL}$ of commercial silica nanospheres in $10 \mathrm{~mL}$ of ethanol solution. The obtained mixture was kept stirring for $20 \mathrm{~min}$. Then $10 \mu \mathrm{L}$ of TEOS and $10 \mathrm{uL}$ of $28 \%$ ammonia 
hydroxide were added into the solution with 20 min intervals. All of the above reactions were carried out under darkness. After overnight incubation, the reaction between TEOS and ammonia hydroxide leads to the formation of a silica shell on the dye-nanoparticle surface. The resulting nanoparticles were collected by washing and re-dispersion steps through centrifuging in ethanol solution for several times. The final nanoparticles were re-suspended in $1 \mathrm{~mL}$ of ethanol solution for future use. This encapsulation method guarantee the uniformity of the dye encapsulated silica nanoparticles and has potential to permit the synthesis of dye encapsulated silica nanoparticles with precisely tunable sizes through adjusting the amount of TEOS and ammonia hydroxide. Particle sizes were determined by Nano Measurer. Figure 6.1a and $\mathbf{b}$ show the SEM images of the commercial silica beads and the synthesized NIRF encapsulated silica nanoparticles respectively. The average particle sizes of the commercial silica beads and the NIRF nanoparticles were found to be $59.6 \pm 2.2 \mathrm{~nm}$ and $64.5 \pm 3.3$ respectively. The increase in size reveals that the thickness of the silica outer layer was at $\sim 5 \mathrm{~nm}$. The fluorescence emission spectrum of the synthesized NIRF nanoparticles is presented in Figure 6.2. The wavelength of the maximum fluorescence emission is at $769 \mathrm{~nm}$.

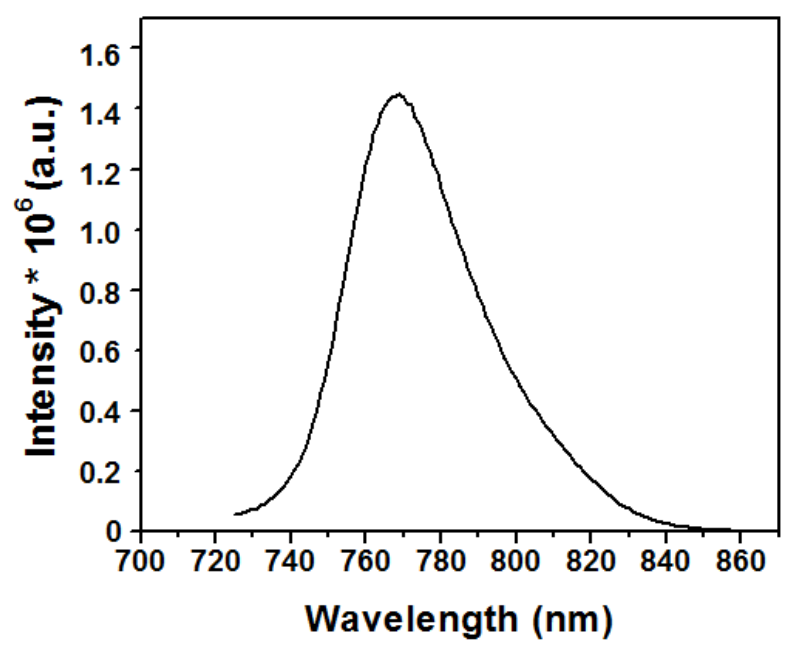

Figure 6.2. Fluorescence emission spectrum of the synthesized NIRF nanoparticles.

\subsubsection{Labeling detection DNA with Nanoparticles}

The preparation detection DNA labeled with NIRF NPs followed the previously reported method. The obtained $1 \mathrm{~mL}$ of NIRF NPs was mixed with $20 \mu \mathrm{L}$ of TEPSA. The mixture solution was incubated overnight to achieve carboxyl group-terminated NIRF NPs. The successful conjugation of carboxyl-group onto the surface of NIRF NPs was confirmed by FT-IR 
spectra (Figure 6.3). After washing with ethanol and D.I. water for several times, the resulting nanoparticles were re-suspended in $0.5 \mathrm{~mL}$ of the solution containing $50 \mathrm{mM} \mathrm{NHS}$ and $200 \mathrm{mM}$ EDC. After incubation for $2 \mathrm{~h}$, the $\mathrm{COOH}$ group was activated. $50 \mu \mathrm{L}$ of $20 \mu \mathrm{M}$ of DNA (amine group labeled detection DNA) solution was then added into the mixture. After overnight incubation, the solution was washed with a buffer solution (PBS containing $0.1 \%$ of BSA) for three times. The resulting nanoparticles were finally suspended in $200 \mu \mathrm{L}$ of Eluent buffer (20 $\mathrm{nM}$ of $\mathrm{Na}_{3} \mathrm{PO}_{4} \cdot 12 \mathrm{H}_{2} \mathrm{O}$ containing $5 \%$ BSA, $0.25 \%$ Tween 20 , and $10 \%$ sucrose) and stored under $4{ }^{\circ} \mathrm{C}$ for future use. The successful conjugation of the detection DNA probe onto the NIRF NPs was confirmed by the FT-IR spectra (Figure 6.4).

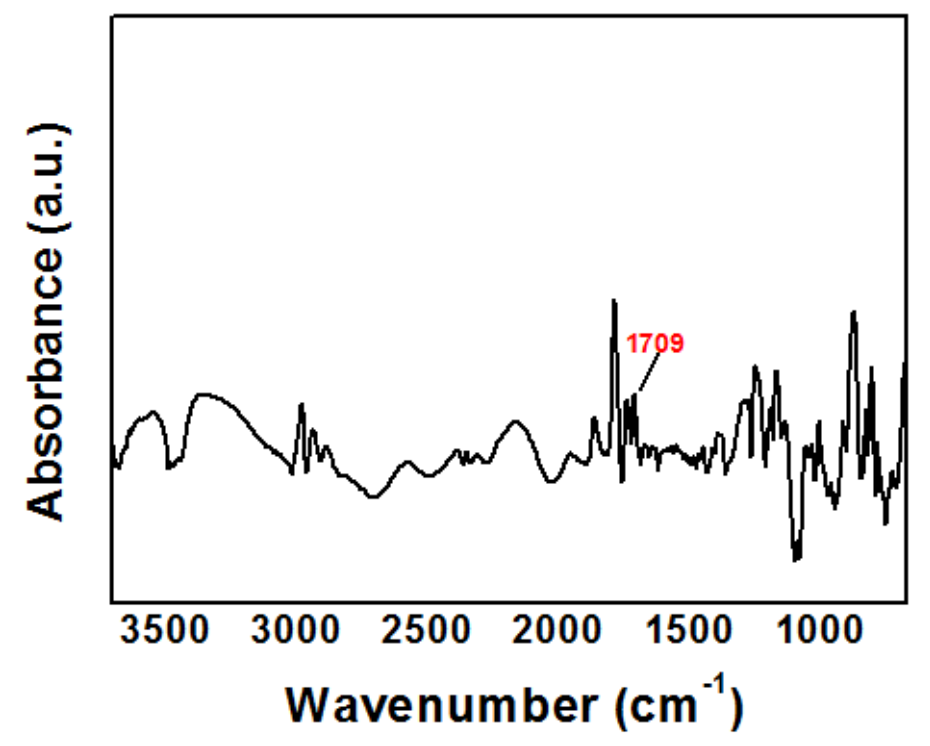

Figure 6.3. FT-IR spectra of the carboxyl-group labeled NIRF NPs. The peak at $1709 \mathrm{~cm}^{-1}$ peak is assigned to the $\mathrm{C}=\mathrm{O}$ stretching vibration in $\mathrm{COOH}$ of TEPSA. 


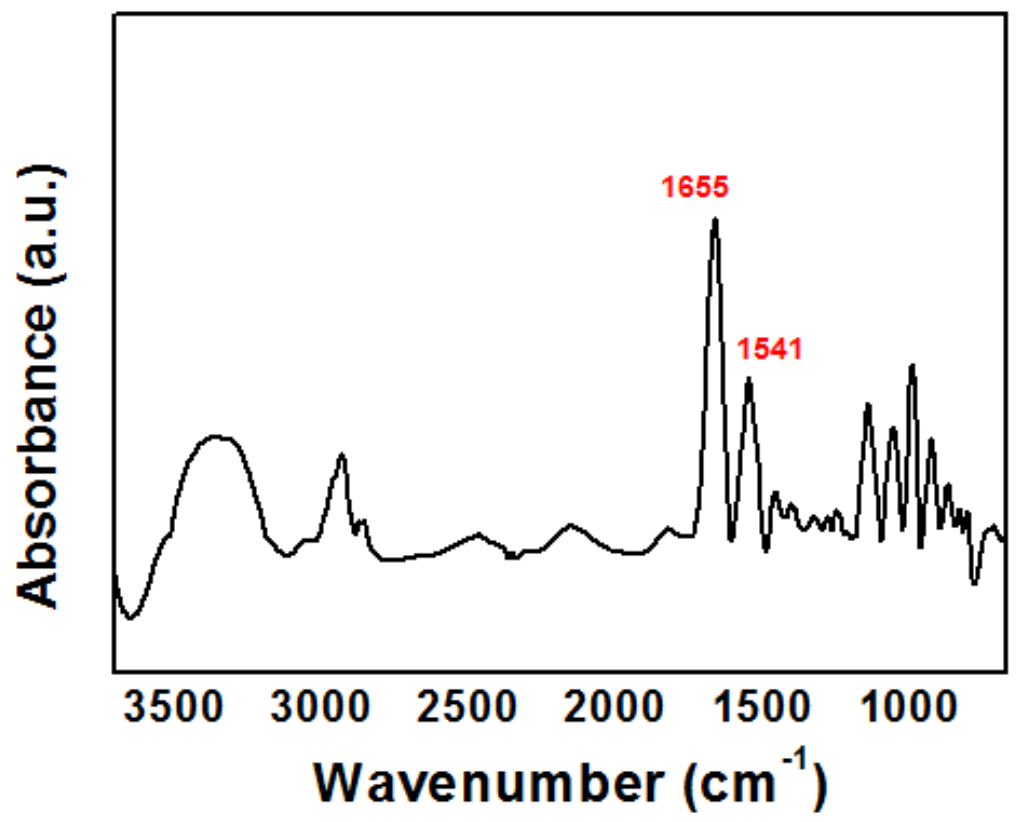

Figure 6.4. FT-IR spectra of the detection DNA probe functionalized NIRF NPs. The peaks at 1655 and $1541 \mathrm{~cm}^{-1}$ : correspond to the $\mathrm{N}-\mathrm{H}$ bending in the $\mathrm{NH}_{2}$ labeled DNA.

\subsubsection{Labeling Biotinylated Detection DNA with Streptavidin}

The biotin-lated detection DNA probe (50 mM) /NIRF NPs was mixed with $200 \mu \mathrm{L}$ of 2.5 $\mathrm{mg} / \mathrm{ml}$ of streptavidin. The mixture was incubated on a shaker for $1 \mathrm{~h}$. The solution was dispensed on the sample pad of the PLFS.

6.2.6 Preparation of Test Strip 


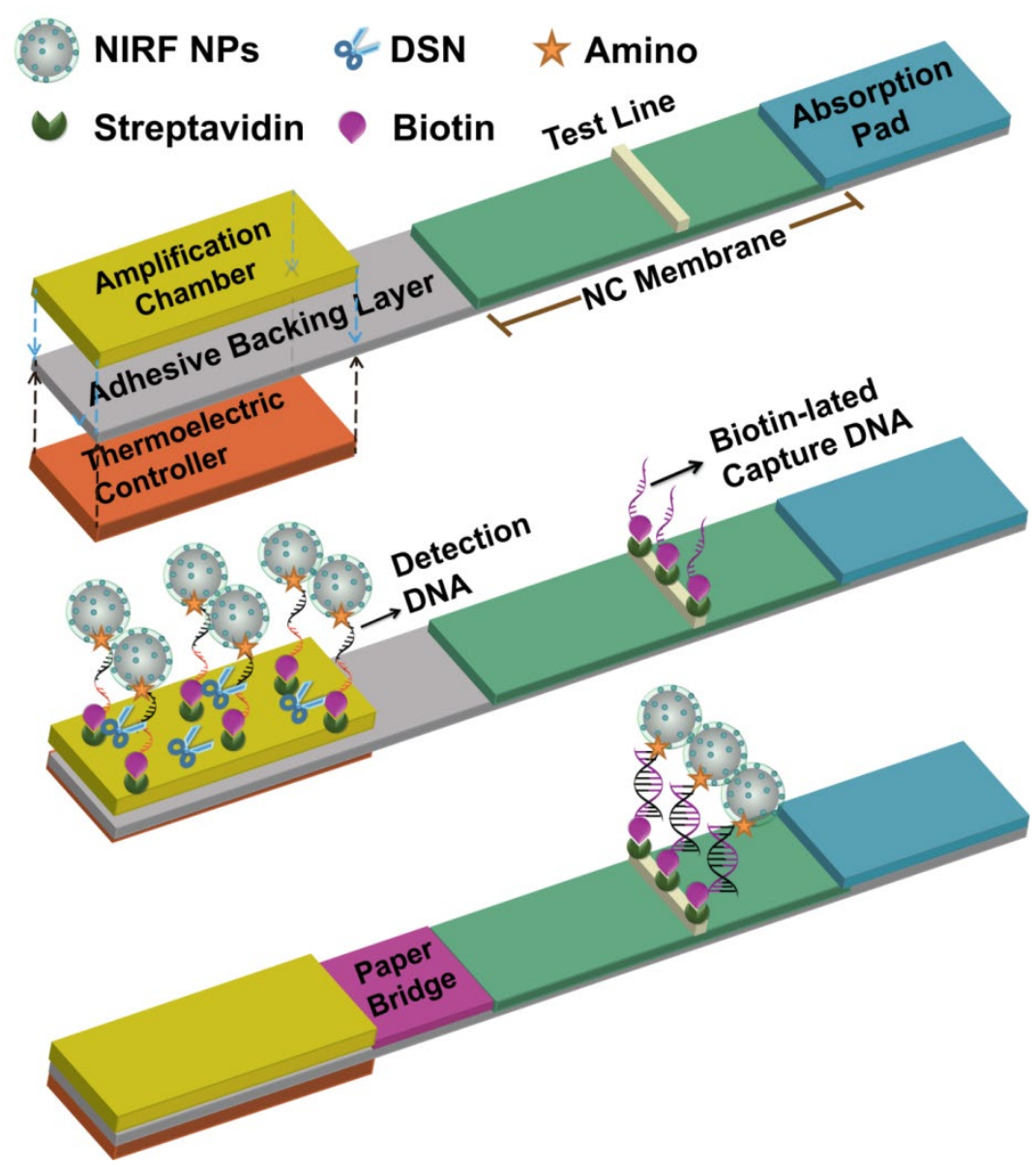

Figure 6.5. Schematic illustration of the DSN-PLFS.

Figure 6.5 exhibits the schematic illustration of the fabricated DSN-PLFS, which consists of five components: a sample pad (reaction chamber), a thermoelectric controller, NC membrane, a paper bridge and an absorption (wick) pad. All the components were mounted on a common backing layer (typically an inert plastic, e.g., polyester). The sample pad was saturated with a Tris- $\mathrm{HCl}$ buffer $(\mathrm{pH} 8.0)$ containing $0.23 \%$ Triton $\mathrm{X}-100$ and 0.05 and $0.15 \mathrm{M} \mathrm{NaCl}$. The pad was then dried at $37{ }^{\circ} \mathrm{C}$ for $2 \mathrm{~h}$ and stored in a desiccator at room temperature (RT). Then, $5 \mu \mathrm{L}$ of NIRF NPs labeled detection DNA probe was dispensed on the samples pad of the PLFS. After the sample pad was dried at $37{ }^{\circ} \mathrm{C}$ for $10 \mathrm{~min}, 0.25 \mathrm{U}$ of DSN was loaded onto it and then the sample pad was dried and stored at $4{ }^{\circ} \mathrm{C}$ under a dry condition. $0.5 \mu \mathrm{L}$ of $1 \mathrm{mg} / \mathrm{mL}$ capture antibodies was dropped onto the $\mathrm{NC}$ membrane to form a test dot. The membrane was then dried and stored at $4{ }^{\circ} \mathrm{C}$ under a dry condition. Finally, the sample pad, NC membrane and the absorption pad were successively assembled onto a plastic adhesive backing layer. The NC 
membrane and absorption pad were overlapped with $2 \mathrm{~mm}$ to ensure that the solution was able to flow across the components. Each strip was cut to be $3 \mathrm{~mm}$ wide by a paper cutter (Swingline 12 in. ClassicCut Lite Trimmer). During the test, the PLFS was soaked into a tube containing 100 $\mu \mathrm{L}$ of sample solution with concentration of target miRNA. Then the tube was incubated in an oven under $60{ }^{\circ} \mathrm{C}$ for $20 \mathrm{~min}$. After reaction, a paper bridge $(8 \mathrm{~mm} \times 3 \mathrm{~mm})$ was placed in the gap between the sample pad the NC membrane, enabling the flow of the sample solution to migrate to the detection area on the NC membrane. After $20 \mathrm{~min}$ reaction, the PLFS was analyzed by a portable fluorescent reader. The fluorescent peak intensity was in proportion to the concentration of target antigen, which was used for quantitative work

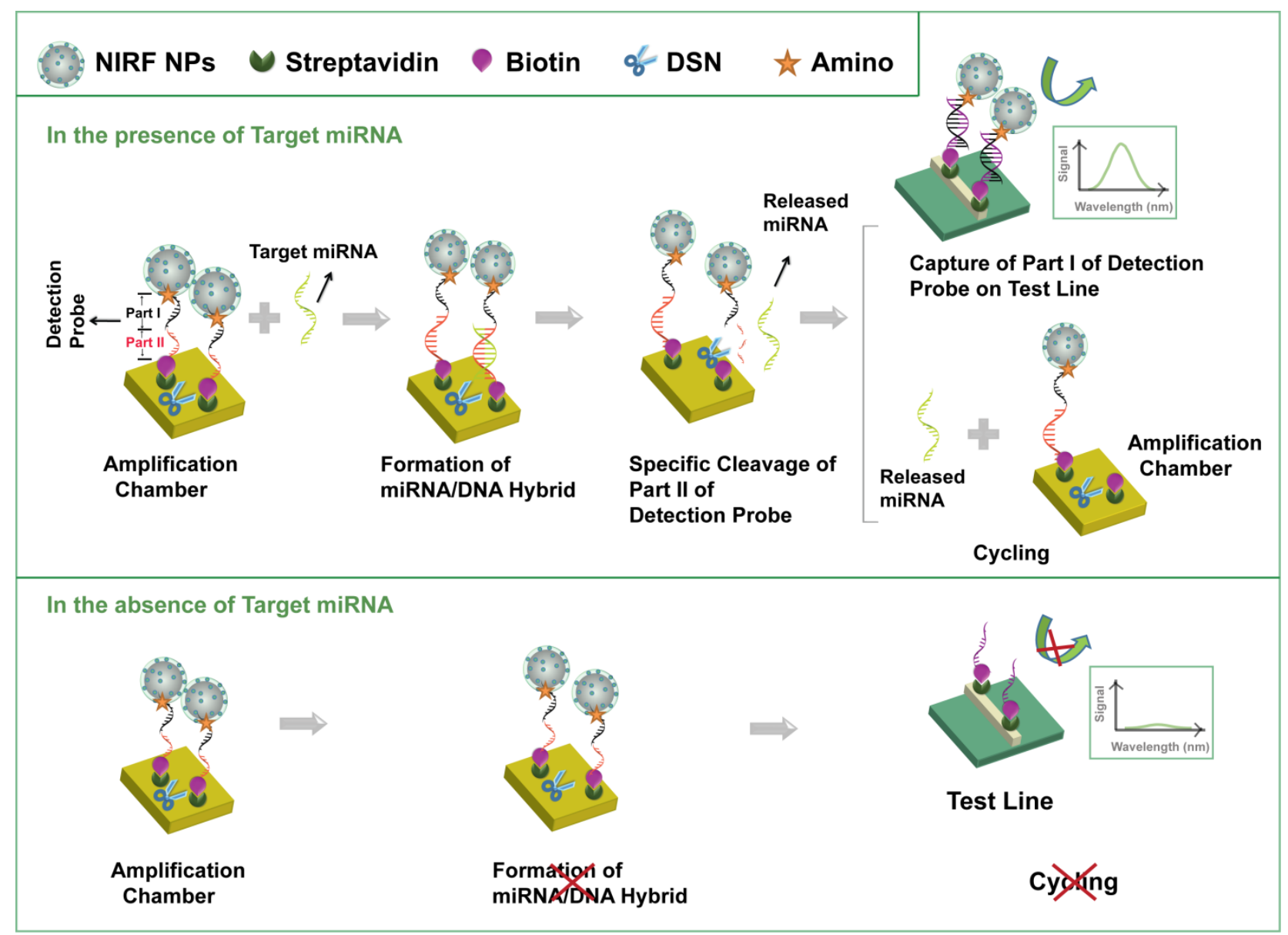

Figure 6.6. Schematic illustration of the signal amplification principle of the DSN-LFTS. 


\subsection{RESULTS AND DISCUSSION}

\subsubsection{Operation Principle}

The procedure of signal amplification of a DSN-PLFS is illustrated in Figure 6.6. As shown in the figure, single strand detection DNA probes labeled NIRF NPs are functionalized on the sample pad. The sequence of the detection DNA probe is specifically designed based on the sequences of the target miRNA and the capture DNA probe. The part II of the detection probe is fully complementary with target miRNA; while the part I is complementary with capture DNA probe on the test area. Upon the addition of target miRNA, it is hybridized with the part II of the detection probe, leading to the formation of miRNA-detection DNA/NIRF NPs heteroduplex on the sample pad. Owing to the present of DSN, the part II DNA probe in the heteroduplex is immediately degrades, at the same time, the target miRNA and the remained part I DNA probe linked NIRF NPs are released. Following the specific cleavage reaction, the released miRNA subsequently hybridizes with the part II probe of the remaining detection DNA to repeat the hybridization/cleavage cycle, leading to the concentration amplification of the NIRF NPs linked DNA probes. The detailed illustration of this amplification procedure is presented in Figure 6.7.

\section{Cycling}

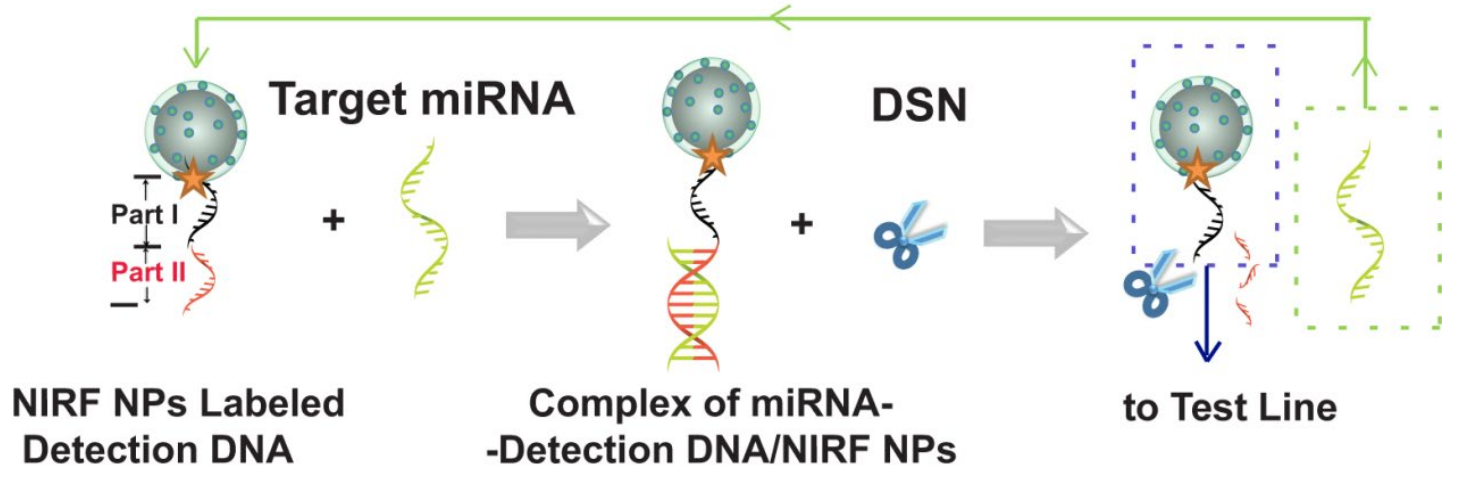

Figure 6.6. Detailed illustration of the signal amplification principle of the DSN-LFTS.

After the amplification reaction, a paper bridge is placed between the sample pad and the NC membrane to guide the liquid migration from the amplification chamber to the $\mathrm{NC}$ membrane, where the capture DNA probe is functionalized. When the solution migrates to the test line, the functionalized capture DNA on the test line can grab the complex of part I DNA probe-NIRF NP through DNA complementary hybridization. At this moment, the NIRF NPs are accumulated on 
the test line and the captured amount is in proportion to the concentration of the target miRNA, which can be employed for quantification work. In the absent of target miRNA, detection DNA linked NIRF NPs cannot be released owing to the fact that DSN is only active towards with the DNA inside of the DNA/RNA heteroduplex but inactive towards single-stranded DNA and RNA. The PLFS is then analyzed by a portable handheld fluorescent strip reader, which has been exhibited in Figure 5.6c.

\subsubsection{Optimizations}
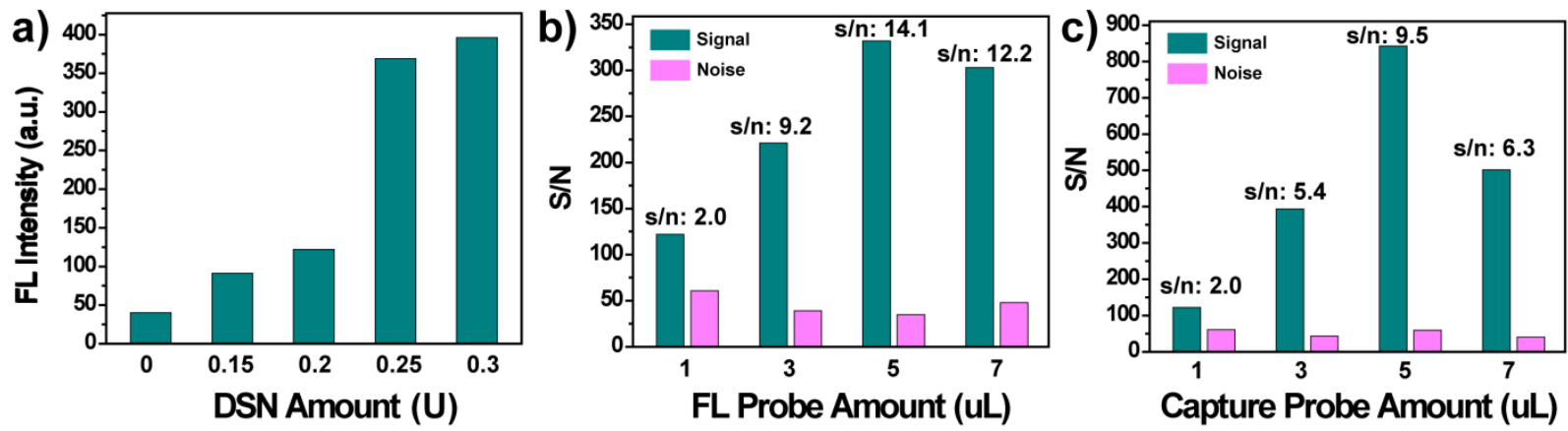

Figure 6.8. Optimization of the experiment conditions for DSN-PLFS. a) Effect of the DSN amount on the fluorescent signals of the PLFSs, b) effect of the NIRF NPs/detection DNA probe on the $\mathrm{S} / \mathrm{N}$ ratio, c) effect of the volume of capture DNA probe on the $\mathrm{S} / \mathrm{N}$ ratio.

In order to optimize the sensor performance, the DSN-PLFS was employed to measure $10 \mathrm{pM}$ of miRNA. Since the DSN significantly influences the hybridization/cleavage reactions and the released amount of NIRF NPs, therefore, the amount of DNS in this amplification system was studied. As shown in Figure 6.8a, the signal intensity of the fluorescent peak increases with the growing amount of DSN until it reaches $0.25 \mathrm{U}$. Further increase in the DSN amount shows little effect on the increase of the peak intensity. Therefore, the optimal amount of DNS was $0.25 \mathrm{U}$. The amount of the detection DNA-NIRF NPs and the concentration of capture DNA probe on the test line are another two important parameters, because both of them affect the ability of DSN-PLFS to capture the NIRF NPs on the test line, which significantly affects the signal intensity and detection sensitivity. As shown in Figure $\mathbf{6 . 8 b}$, c, the optimal amount of the detection DNA-NIRF NPs was $5 \mu \mathrm{L}$, and $5 \mu \mathrm{L}$ of the capture DNA probe was the best. 

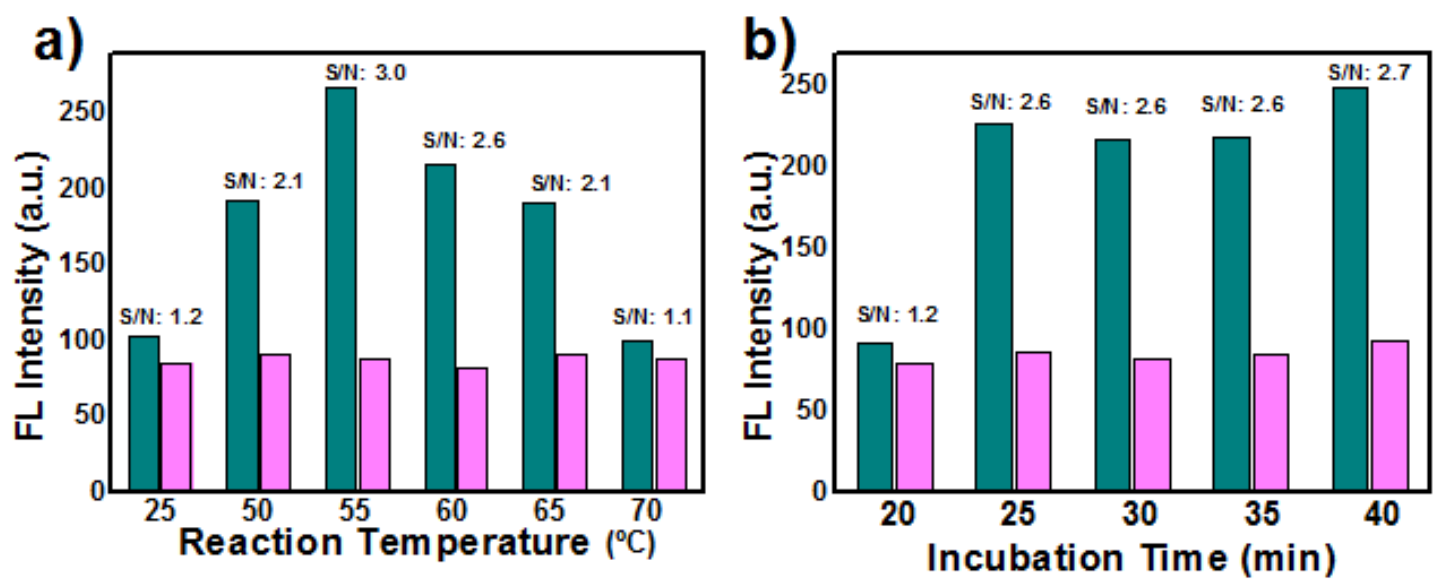

Figure 6.9. Optimization of the experiment conditions for DSN-PLFS. a) Effect of the on the reaction temperature of the DSN-PLFSs, b) effect of the reaction time on the $\mathrm{S} / \mathrm{N}$ ratio of the DSN-PLFSs.

The optimal temperature of DSN-PLFS was determined tuning reaction temperature varying from $25{ }^{\circ} \mathrm{C}$ to $70^{\circ} \mathrm{C}$. Under the conditions employed, the highest $\mathrm{S} / \mathrm{N}$ ratio was observed at $55^{\circ} \mathrm{C}$. Further increases in temperature were accompanied by a signal decrease (Figure 6.9a). This might reflect enzyme denaturation. We also evaluated the effect of incubation time of enzyme amplification reaction on $\mathrm{S} / \mathrm{N}$ ratio (Figure 6.9b). We found that increases in incubation time were accompanied by increases in $\mathrm{S} / \mathrm{N}$ ratio until incubation time reached $25 \mathrm{~min}$. After $25 \mathrm{~min}$, $\mathrm{S} / \mathrm{N}$ ratios were remained constant. Therefore, the optimal incubation time of the enzyme amplification reaction was $25 \mathrm{~min}$

\subsubsection{Performance in Running Buffer}




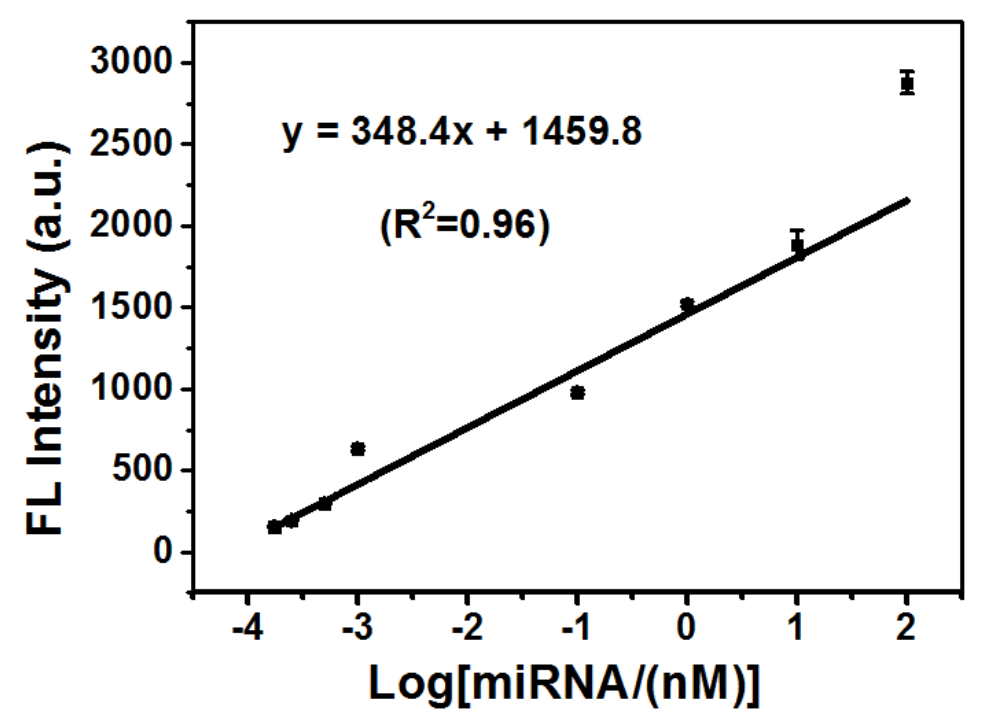

Figure 6.10. Peak intensity of the fluorescent signal obtained from the DSN-PLFS in running buffer with different concentrations of miRNA (ranging from 0 to $100 \mathrm{nM}$ ).

In order to further evaluate the performances of the DSN-PLFS, sample solutions containing different concentrations of miRNA-34 (ranging from 0 to $100 \mathrm{nM}$ ) were measured under optimal experimental conditions. During a typical test, a DSN-PLFS was soaked into a tube which contains $100 \mu \mathrm{L}$ of sample solution. After the amplification reaction in the oven for 20 min and the reaction of the capturing NIRF NPS on the test line for another $20 \mathrm{~min}$, the fluorescent response of the DSN-PLFS was recorded. As descripted in Figure 6.10, the fluorescent intensity increases with the increasing of miRNA concentration, which indicates that the DSN-PLFS is working properly. The calibration curve showed a linear range from $175 \mathrm{fM}$ to $100 \mathrm{nM}$. The detection limit of the DSN-PLFS for target miRNA was found to be $150 \mathrm{fM}$, which was estimated based on three times of signal-to-noise ratio. Compared with the sensitivity of NIRFPLFS in Chapter 5, an improvement in sensitivity of three-orders of magnetite was obtained by the developed DSN-PLFS for miRNA measurement in running buffer samples.

\subsubsection{Performance in 50\% of Blood Plasma}




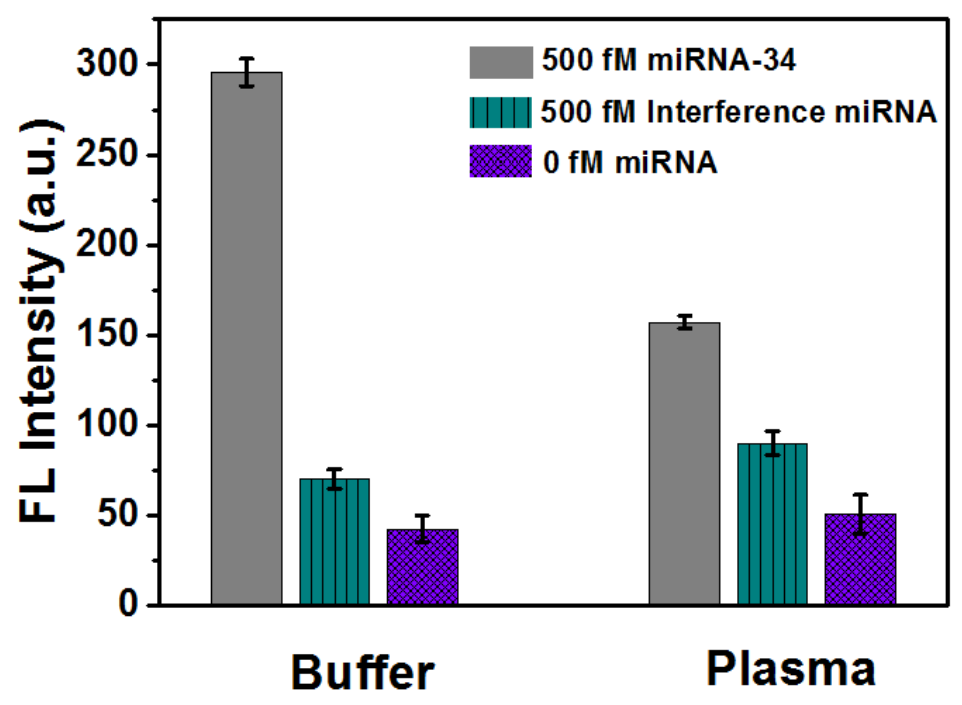

Figure 6.11. The fluorescent intensities of the DSN-PLFS test zones in the presence of $500 \mathrm{fM}$ of miRNA-34, $500 \mathrm{fM}$ of interference miRNA and $0 \mathrm{fM}$ of miRNA in running buffer and $50 \%$ of plasma.

Anti-interference ability is an important parameter to evaluate a biosensor. Before the test was conducted in real plasma samples, selectivity test was carried out by employing miRNA molecule with two mismatches of bases towards target miRNA-34. As demonstrated in Figure 6.11, compared with the target signals, signals from the interference tests are comparable with the signals generated from control experiments and can be negligible, indicating that the developed DSN-PLFS exhibits good selectivity towards interference miRNA molecules. The intrinsic advantages can merit miRNA detection in complex real samples.

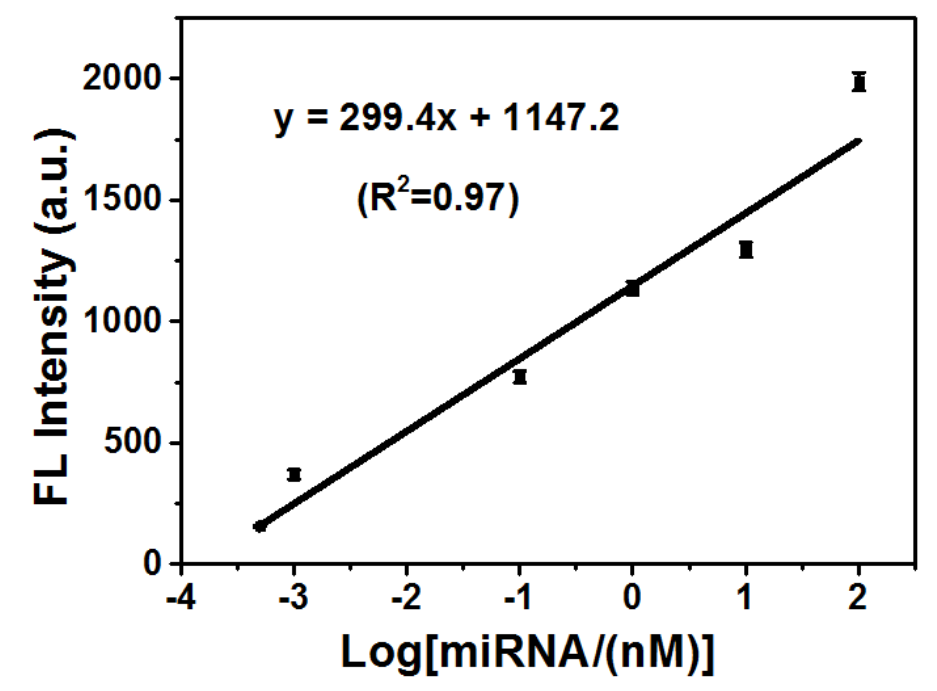


Figure 6.12. Peak intensity of the fluorescent signal obtained from the DSN-PLFS in $50 \%$ of blood plasma with different concentrations of miRNA (ranging from 0 to $100 \mathrm{nM}$ ).

The performance of the DSN-PLFS was then studied in the complex human fluids. $50 \%$ of plasma sample solutions containing different concentrations of miRNA-34 (ranging from 0 to $100 \mathrm{nM}$ ) were measured under optimal experimental conditions. After the reactions, the fluorescent response of the DSN-PLFS was recorded by a portable fluorescent reader. As descripted in Figure 6.12, the fluorescent intensity increases with the increasing of miRNA concentration. The fluoresce cent peak intensity (y) versus the miRNA-34 concentration (x) was fitted as $y=311.2 x+1198.4$ with the relative coefficient $\left(R^{2}\right)$ of 0.95 , showing a linear detection range from $500 \mathrm{fM}$ to $100 \mathrm{nM}$, achieving a LOD of $300 \mathrm{fM}$. The LOD was estimated according to three times of signal-to-noise ratio. Compared with the sensitivity of NIRF-PLFS in Chapter 5, an improvement in sensitivity of two orders of magnitude was obtained by the developed DSN-PLFS for miRNA measurement in running buffer samples. Our experimental results show the DSN-PLFS developed in the present work can work for miRNA-34 detection in blood plasma.

\subsubsection{Measurement of miRNA-34 Biomarker in Clinical Blood Plasma Samples}

The calibrated DSN-PLFS was employed to measure the miRNA-34 biomarker level in clinical blood plasma taken from stroke patients. Figure $\mathbf{6 . 1 3}$ shows the miRNA-34 concentrations in six clinical blood plasma samples measured by the DSN-PLFS. The results indicated that the developed DSN-PLFS can work properly in clinical patient samples and can meet the demands for measuring trace amount of miRNA biomarkers directly from clinical samples. Compared with clinical standard PCR approach, the developed DSN-PLFS exhibits advantages in simplicity, shorter reaction time, low cost and used friendly. 


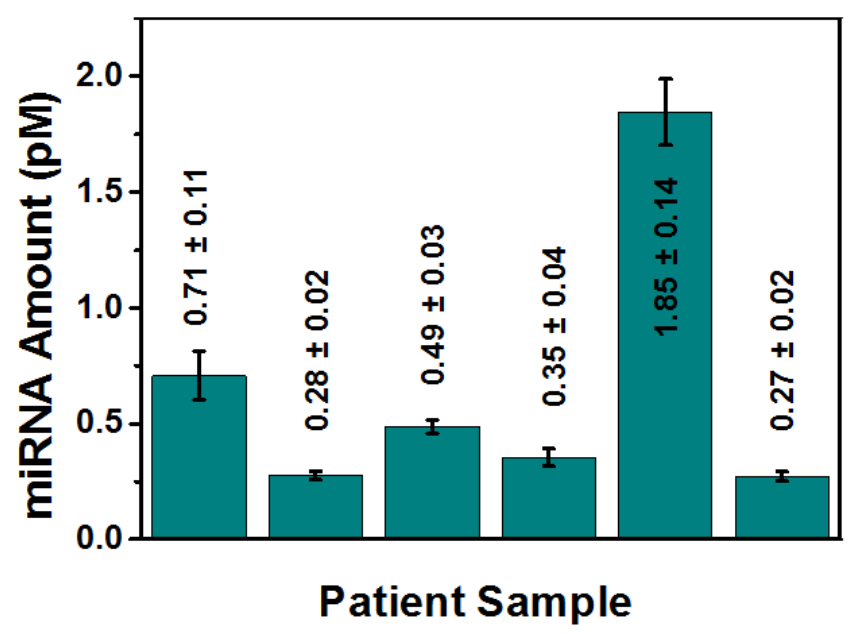

Figure 6.13. miRNA-34 concentrations in clinical blood plasma samples, which were measured by DSN-PLFS.

\subsection{CONCLUSION}

The DSN-PLFS was developed for ultrasensitive measurement of stroke biomarker microRNA-34 in blood plasma samples. DSN assisted amplification technique was employed to modify the PLFS for improving the sensor performance. After signal amplification, the DSNPLFS can amplify fluorescent response, leading to an improvement in detection sensitivity with two-orders of magnitude. The obtained LOD was below the cut-off value that presents in the blood samples of stroke patients. Moreover, the DSN-PLFS shows advantages over the standard PCR approach, including low cost, portability, short operation time, and simple operation procedure. These merits endow the DSN-PLFS with great potential to be employed as a POC tool for monitoring the miRNA biomarkers in biological samples. The DSN-PLFS was successfully employed for detection of miRNA-34a in clinical blood plasma samples. Obviously, the DSN-PLFS has advantages over the standard PCR approach, including low cost, portability and short operation time. These merits endow the DSN-PLFS with great potential to be employed as a POC tool for monitoring the miRNA biomarkers in real-world samples. 


\section{Chapter 7 CONCLUSION AND OUTLOOK}

This dissertation summarized the background, recent advances and obstacles of PLFS devices for disease biomarker detections. In order to solve the problems and fill the gaps between the PLFSs and the practical POC devices, several strategies have been proposed:

1) Two parallel routes of ultrasensitive techniques: SERS and NIR fluorescence techniques have been employed in PLFSs to build proof-of-concept sensors and improve detection sensitivity;

2) Synthesized SERS and NIRF nanomaterials with silica coating layers to protect the nanomaterials from the interference from the surrounding environment, leading to the improvement in anti-interference capacity;

3) Tuned the SERS and NIRF nanomaterials to the near-infrared window to reduce the interference signals from surrounding environment and biological matrices;

4) Fabricated blood plasma separation unit (PSU) to reduce the interference from the RBCs from whole blood samples;

5) Integrated SERS-PLFS with plasmonic chip to form 3D confined plamonic field for further improving signal intensity and sensitivity;

6) Modified PLFSs with duplex specific nuclease (DSN)-mediated signal amplification strategy for improving detection sensitivity.

As a result, the developed PLFSs in this dissertation can realize accurate and direct measurement of biomarkers from clinical samples, showing great advantages as a practical POC device. Despite the progress that has been achieved in this dissertation, there still exist some issues to be solved. (i) Although the proposed PLFS has realized disease biomarker detection from clinical samples, it is still difficult to employ PLFS to indicate a specific disease if only one biomarker is detected during one trial. Therefore, a high-throughput PLFS which enables multiple biomarker measurements can substantially improve the diagnostic specificity. (ii) Compared with paper materials, the employed blood plasma filtration membrane in this dissertation is relatively more expensive. In order to further decrease the cost of the device, other less-expensive materials, such as porous polymers or general filter paper might provide alternative choices for filtrating plasma from whole blood samples. (iii) During one PLFS test, at least $100 \mu \mathrm{L}$ of aqueous solutions or clinical samples is required, which is not applicable for the 
applications of miniscule amounts of samples and reagents. The future innovative research is expected to introduce microfluidic technique with PLFS through fabricating microfluidic channels on PLFS surfaces through wax-printing technique to solve this problem. 


\section{Reference:}

1. Chin, C. D.; Laksanasopin, T.; Cheung, Y. K.; Steinmiller, D.; Linder, V.; Parsa, H.; Wang, J.; Moore, H.; Rouse, R.; Umviligihozo, G., Microfluidics-based diagnostics of infectious diseases in the developing world. Nature medicine 2011, 17 (8), 1015.

2. $\quad$ Ahn, C. H.; Choi, J.-W.; Beaucage, G.; Nevin, J. H.; Lee, J.-B.; Puntambekar, A.; Lee, J. Y., Disposable smart lab on a chip for point-of-care clinical diagnostics. Proceedings of the IEEE 2004, 92 (1), 154-173.

3. Drain, P. K.; Hyle, E. P.; Noubary, F.; Freedberg, K. A.; Wilson, D.; Bishai, W. R.; Rodriguez, W.; Bassett, I. V., Diagnostic point-of-care tests in resource-limited settings. The Lancet infectious diseases 2014, 14 (3), 239-249.

4. Gao, X.; Xu, L.-P.; Zhou, S.-F.; Liu, G.; Zhang, X., Recent Advances in Nanoparticlesbased Lateral Flow Biosensors. American Journal of Biomedical Sciences 2014, 6 (1).

5. Xiao, Y.; Patolsky, F.; Katz, E.; Hainfeld, J. F.; Willner, I., " Plugging into enzymes": Nanowiring of redox enzymes by a gold nanoparticle. Science 2003, 299 (5614), 1877-1881.

6. Putzbach, W.; Ronkainen, N. J., Immobilization techniques in the fabrication of nanomaterial-based electrochemical biosensors: A review. Sensors 2013, 13 (4), 4811-4840.

7. Gole, A.; Dash, C.; Ramakrishnan, V.; Sainkar, S.; Mandale, A.; Rao, M.; Sastry, M., Pepsin- gold colloid conjugates: preparation, characterization, and enzymatic activity. Langmuir 2001, 17 (5), 1674-1679.

8. Yu, C.; Irudayaraj, J., Multiplex biosensor using gold nanorods. Analytical chemistry 2007, 79 (2), 572-579.

9. (a) Lee, S.; Pérez-Luna, V. H., Dextran- gold nanoparticle hybrid material for biomolecule immobilization and detection. Analytical chemistry 2005, 77 (22), 7204-7211; (b) Thaxton, C. S.; Georganopoulou, D. G.; Mirkin, C. A., Gold nanoparticle probes for the detection of nucleic acid targets. Clinica Chimica Acta 2006, 363 (1-2), 120-126.

10. (a) Abbas, A.; Linman, M. J.; Cheng, Q., New trends in instrumental design for surface plasmon resonance-based biosensors. Biosensors and Bioelectronics 2011, 26 (5), 1815-1824; (b) Hossain, M. K.; Kitahama, Y.; Huang, G. G.; Han, X.; Ozaki, Y., Surface-enhanced Raman scattering: realization of localized surface plasmon resonance using unique substrates and methods. Analytical and bioanalytical chemistry 2009, 394 (7), 1747-1760.

11. (a) Wang, W.; Wu, W.-Y.; Zhong, X.; Miao, Q.; Zhu, J.-J., Aptamer-based PDMS-gold 
nanoparticle composite as a platform for visual detection of biomolecules with silver enhancement. Biosensors and Bioelectronics 2011, 26 (7), 3110-3114; (b) Wen, J.; Zhou, S.; Yuan, Y., Graphene oxide as nanogold carrier for ultrasensitive electrochemical immunoassay of Shewanella oneidensis with silver enhancement strategy. Biosensors and Bioelectronics 2014, 52, 44-49; (c) Yang, W.; Li, X.-b.; Liu, G.-w.; Zhang, B.-b.; Zhang, Y.; Kong, T.; Tang, J.-j.; Li, D.-n.; Wang, Z., A colloidal gold probe-based silver enhancement immunochromatographic assay for the rapid detection of abrin-a. Biosensors and Bioelectronics 2011, 26 (8), 3710-3713.

12. Gao, X.; Xu, L.-P.; Wu, T.; Wen, Y.; Ma, X.; Zhang, X., An enzyme-amplified lateral flow strip biosensor for visual detection of microRNA-224. Talanta 2016, 146, 648-654.

13. (a) Dong, H.; Zhang, J.; Ju, H.; Lu, H.; Wang, S.; Jin, S.; Hao, K.; Du, H.; Zhang, X., Highly sensitive multiple microRNA detection based on fluorescence quenching of graphene oxide and isothermal strand-displacement polymerase reaction. Analytical chemistry 2012, 84 (10), 4587-4593; (b) He, Y.; Zeng, K.; Zhang, S.; Gurung, A. S.; Baloda, M.; Zhang, X.; Liu, G., Visual detection of gene mutations based on isothermal strand-displacement polymerase reaction and lateral flow strip. Biosensors and Bioelectronics 2012, 31 (1), 310-315.

14. Resch-Genger, U.; Grabolle, M.; Cavaliere-Jaricot, S.; Nitschke, R.; Nann, T., Quantum dots versus organic dyes as fluorescent labels. Nature methods 2008, 5 (9), 763.

15. Smith, A. M.; Nie, S., Next-generation quantum dots. Nature biotechnology 2009, 27 (8), 732.

16. Li, Z.; Wang, Y.; Wang, J.; Tang, Z.; Pounds, J. G.; Lin, Y., Rapid and sensitive detection of protein biomarker using a portable fluorescence biosensor based on quantum dots and a lateral flow test strip. Analytical chemistry 2010, 82 (16), 7008-7014.

17. Kam, N. W. S.; O'Connell, M.; Wisdom, J. A.; Dai, H., Carbon nanotubes as multifunctional biological transporters and near-infrared agents for selective cancer cell destruction. Proceedings of the National Academy of Sciences 2005, 102 (33), 11600-11605.

18. Wu, X.; Tian, F.; Wang, W.; Chen, J.; Wu, M.; Zhao, J. X., Fabrication of highly fluorescent graphene quantum dots using L-glutamic acid for in vitro/in vivo imaging and sensing. Journal of Materials Chemistry C 2013, 1 (31), 4676-4684.

19. (a) Hsieh, B.-Y.; Chang, Y.-F.; Ng, M.-Y.; Liu, W.-C.; Lin, C.-H.; Wu, H.-T.; Chou, C., Localized surface plasmon coupled fluorescence fiber-optic biosensor with gold nanoparticles. Analytical Chemistry 2007, 79 (9), 3487-3493; (b) Wang, Y.; Bao, L.; Liu, Z.; Pang, D.-W., 
Aptamer biosensor based on fluorescence resonance energy transfer from upconverting phosphors to carbon nanoparticles for thrombin detection in human plasma. Analytical chemistry 2011, 83 (21), 8130-8137.

20. Li, C.; Luo, W.; Xu, H.; Zhang, Q.; Xu, H.; Aguilar, Z. P.; Lai, W.; Wei, H.; Xiong, Y., Development of an immunochromatographic assay for rapid and quantitative detection of clenbuterol in swine urine. Food Control 2013, 34 (2), 725-732.

21. Qin, Z.; Chan, W. C.; Boulware, D. R.; Akkin, T.; Butler, E. K.; Bischof, J. C., Significantly improved analytical sensitivity of lateral flow immunoassays by using thermal contrast. Angewandte Chemie International Edition 2012, 51 (18), 4358-4361.

22. Zhang, Z.; Bast, R. C.; Yu, Y.; Li, J.; Sokoll, L. J.; Rai, A. J.; Rosenzweig, J. M.; Cameron, B.; Wang, Y. Y.; Meng, X.-Y., Three biomarkers identified from serum proteomic analysis for the detection of early stage ovarian cancer. Cancer research 2004, 64 (16), 5882 5890.

23. Wang, Y.-K.; Yan, Y.-X.; Ji, W.-H.; Wang, H.-a.; Li, S.-Q.; Zou, Q.; Sun, J.-H., Rapid simultaneous quantification of zearalenone and fumonisin B1 in corn and wheat by lateral flow dual immunoassay. Journal of agricultural and food chemistry 2013, 61 (21), 5031-5036.

24. (a) Smith, W., Practical understanding and use of surface enhanced Raman scattering/surface enhanced resonance Raman scattering in chemical and biological analysis. Chemical Society Reviews 2008, 37 (5), 955-964; (b) Willets, K. A., Surface-enhanced Raman scattering (SERS) for probing internal cellular structure and dynamics. Analytical and bioanalytical chemistry 2009, 394 (1), 85-94.

25. (a) Porter, M. D.; Lipert, R. J.; Siperko, L. M.; Wang, G.; Narayanan, R., SERS as a bioassay platform: fundamentals, design, and applications. Chemical Society Reviews 2008, 37 (5), 1001-1011; (b) Schlücker, S., SERS microscopy: nanoparticle probes and biomedical applications. ChemPhysChem 2009, 10 (9-10), 1344-1354.

26. Liu, H.; Zhang, L.; Lang, X.; Yamaguchi, Y.; Iwasaki, H.; Inouye, Y.; Xue, Q.; Chen, M., Single molecule detection from a large-scale SERS-active Au 79 Ag 21 substrate. Scientific Reports 2011, 1, 112 .

27. (a) Medina, M. A.; Schwille, P., Fluorescence correlation spectroscopy for the detection and study of single molecules in biology. Bioessays 2002, 24 (8), 758-764; (b) Moschou, E. A.; Sharma, B. V.; Deo, S. K.; Daunert, S., Fluorescence glucose detection: advances toward the 
ideal in vivo biosensor. Journal of fluorescence 2004, 14 (5), 535-547; (c) Weiss, S., Fluorescence spectroscopy of single biomolecules. Science 1999, 283 (5408), 1676-1683.

28. Medintz, I. L.; Clapp, A. R.; Brunel, F. M.; Tiefenbrunn, T.; Uyeda, H. T.; Chang, E. L.; Deschamps, J. R.; Dawson, P. E.; Mattoussi, H., Proteolytic activity monitored by fluorescence resonance energy transfer through quantum-dot-peptide conjugates. Nature materials 2006, 5 (7), 581 .

29. Becker, A.; Hessenius, C.; Licha, K.; Ebert, B.; Sukowski, U.; Semmler, W.; Wiedenmann, B.; Grötzinger, C., Receptor-targeted optical imaging of tumors with near-infrared fluorescent ligands. Nature biotechnology 2001, 19 (4), 327.

30. Hemmer, E.; Benayas, A.; Légaré, F.; Vetrone, F., Exploiting the biological windows: current perspectives on fluorescent bioprobes emitting above $1000 \mathrm{~nm}$. Nanoscale Horizons 2016, 1 (3), 168-184.

31. Yan, J.; Estévez, M. C.; Smith, J. E.; Wang, K.; He, X.; Wang, L.; Tan, W., Dye-doped nanoparticles for bioanalysis. Nano today 2007, 2 (3), 44-50.

32. Mondello, S.; Muller, U.; Jeromin, A.; Streeter, J.; Hayes, R. L.; Wang, K. K., Bloodbased diagnostics of traumatic brain injuries. Expert review of molecular diagnostics 2011, 11 (1), 65-78.

33. Lee, B.; Newberg, A., Neuroimaging in traumatic brain imaging. NeuroRx 2005, 2 (2), 372-383.

34. HERRMANN, M.; JOST, S.; KUTZ, S.; EBERT, A. D.; KRATZ, T.; WUNDERLICH, M. T.; SYNOWITZ, H., Temporal profile of release of neurobiochemical markers of brain damage after traumatic brain injury is associated with intracranial pathology as demonstrated in cranial computerized tomography. Journal of neurotrauma 2000, 17 (2), 113-122.

35. Yu, X.; Wang, Y.; Chen, X.; Wu, K.; Chen, D.; Ma, M.; Huang, Z.; Wu, W.; Li, C., Whitelight-exciting, layer-by-layer-assembled $\mathrm{ZnCdHgSe}$ quantum dots/polymerized ionic liquid hybrid film for highly sensitive photoelectrochemical immunosensing of neuron specific enolase. Analytical chemistry 2015, 87 (8), 4237-4244.

36. Fu, X.; Meng, M.; Zhang, Y.; Yin, Y.; Zhang, X.; Xi, R., Chemiluminescence enzyme immunoassay using magnetic nanoparticles for detection of neuron specific enolase in human serum. Analytica chimica acta 2012, 722, 114-118.

37. Cheng, S.; Hideshima, S.; Kuroiwa, S.; Nakanishi, T.; Osaka, T., Label-free detection of 
tumor markers using field effect transistor (FET)-based biosensors for lung cancer diagnosis. Sensors and Actuators B: Chemical 2015, 212, 329-334.

38. Gao, X.; Xu, H.; Baloda, M.; Gurung, A. S.; Xu, L.-P.; Wang, T.; Zhang, X.; Liu, G., Visual detection of microRNA with lateral flow nucleic acid biosensor. Biosensors and Bioelectronics 2014, 54, 578-584.

39. Lin, Y.-Y.; Wang, J.; Liu, G.; Wu, H.; Wai, C. M.; Lin, Y., A nanoparticle label/immunochromatographic electrochemical biosensor for rapid and sensitive detection of prostate-specific antigen. Biosensors and Bioelectronics 2008, 23 (11), 1659-1665.

40. Li, M.; Cushing, S. K.; Zhang, J.; Suri, S.; Evans, R.; Petros, W. P.; Gibson, L. F.; Ma, D.; Liu, Y.; Wu, N., Three-dimensional hierarchical plasmonic nano-architecture enhanced surfaceenhanced Raman scattering immunosensor for cancer biomarker detection in blood plasma. $A C S$ nano 2013, 7 (6), 4967-4976.

41. (a) Fu, X.; Cheng, Z.; Yu, J.; Choo, P.; Chen, L.; Choo, J., A SERS-based lateral flow assay biosensor for highly sensitive detection of HIV-1 DNA. Biosensors and Bioelectronics 2016, 78, 530-537; (b) Liang, J.; Liu, H.; Lan, C.; Fu, Q.; Huang, C.; Luo, Z.; Jiang, T.; Tang, Y., Silver nanoparticle enhanced Raman scattering-based lateral flow immunoassays for ultrasensitive detection of the heavy metal chromium. Nanotechnology 2014, 25 (49), 495501; (c) Wang, X.; Choi, N.; Cheng, Z.; Ko, J.; Chen, L.; Choo, J., Simultaneous detection of dual nucleic acids using a SERS-based lateral flow assay biosensor. Analytical chemistry 2016, 89 (2), 1163 1169.

42. Li, M.; Cushing, S. K.; Zhang, J.; Lankford, J.; Aguilar, Z. P.; Ma, D.; Wu, N., Shapedependent surface-enhanced Raman scattering in gold-Raman-probe-silica sandwiched nanoparticles for biocompatible applications. Nanotechnology 2012, 23 (11), 115501.

43. Gao, X.; Zheng, P.; Kasani, S.; Wu, S.; Yang, F.; Lewis, S.; Nayeem, S.; EnglerChiurazzi, E. B.; Wigginton, J. G.; Simpkins, J. W., Based surface-enhanced Raman scattering lateral flow strip for detection of neuron-specific enolase in blood plasma. Analytical chemistry 2017, 89 (18), 10104-10110.

44. (a) He, Y.; Wu, Y.; Fu, J.-Z.; Wu, W.-B., Fabrication of paper-based microfluidic analysis devices: a review. Rsc Advances 2015, 5 (95), 78109-78127; (b) Martinez, A. W.; Phillips, S. T.; Whitesides, G. M.; Carrilho, E., Diagnostics for the developing world: microfluidic paper-based analytical devices. ACS Publications: 2009; (c) Siegel, A. C.; Phillips, S. T.; Dickey, M. D.; Lu, 
N.; Suo, Z.; Whitesides, G. M., Foldable printed circuit boards on paper substrates. Advanced Functional Materials 2010, 20 (1), 28-35.

45. Nakashima, Y.; Hata, S.; Yasuda, T., Blood plasma separation and extraction from a minute amount of blood using dielectrophoretic and capillary forces. Sensors and Actuators B: Chemical 2010, 145 (1), 561-569.

46. (a) Dean, L., Blood groups and red cell antigens. National Center for Biotechnology Information: 2005; (b) Li, H.; Han, D.; Pauletti, G.; Steckl, A., Blood coagulation screening using a paper-based microfluidic lateral flow device. Lab on a Chip 2014, 14 (20), 4035-4041.

47. (a) Ataullakhanov, F.; Pohilko, A.; Sinauridze, E.; Volkova, R., Calcium threshold in human plasma clotting kinetics. Thrombosis research 1994, 75 (4), 383-394; (b) Nilghaz, A.; Shen, W., Low-cost blood plasma separation method using salt functionalized paper. Rsc Advances 2015, 5 (66), 53172-53179.

48. Li, M.; Cushing, S. K.; Zhang, J. M.; Lankford, J.; Aguilar, Z. P.; Ma, D. L.; Wu, N. Q., Shape-dependent surface-enhanced Raman scattering in gold-Ramanprobe-silica sandwiched nanoparticles for biocompatible applications. Nanotechnology 2012, 23 (11).

49. (a) Beauchemin, N.; Arabzadeh, A., Carcinoembryonic antigen-related cell adhesion molecules (CEACAMs) in cancer progression and metastasis. Cancer and Metastasis Reviews 2013, 32 (3-4), 643-671; (b) Grunnet, M.; Sorensen, J., Carcinoembryonic antigen (CEA) as tumor marker in lung cancer. Lung cancer 2012, 76 (2), 138-143.

50. (a) Minton, J. P.; Hoehn, J. L.; Gerber, D. M.; Horsley, J. S.; Connolly, D. P.; Salwan, F.; Fletcher, W. S.; Cruz Jr, A. B.; Gatchell, F. G.; Oviedo, M., Results of a 400-patient carcinoembryonic antigen second-look colorectal cancer study. Cancer 1985, 55 (6), 1284-1290; (b) Moertel, C. G.; O'Fallon, J. R.; Go, V. L.; O'Connell, M. J.; Thynne, G. S., The preoperative carcinoembryonic antigen test in the diagnosis, staging, and prognosis of colorectal cancer. Cancer 1986, 58 (3), 603-610.

51. (a) Bogue, R., MEMS sensors: past, present and future. Sensor Review 2007, 27 (1), 713; (b) St John, A.; Price, C. P., Existing and emerging technologies for point-of-care testing. The Clinical Biochemist Reviews 2014, 35 (3), 155; (c) Windmiller, J. R.; Wang, J., Wearable electrochemical sensors and biosensors: a review. Electroanalysis 2013, 25 (1), 29-46.

52. $\quad$ (a) Lateral Flow Immunoassay. 2009; p 1-223; (b) Hu, J.; Wang, S. Q.; Wang, L.; Li, F.; Pingguan-Murphy, B.; Lu, T. J.; Xu, F., Advances in paper-based point-of-care diagnostics. 
Biosensors \& Bioelectronics 2014, 54, 585-597; (c) Quesada-Gonzalez, D.; Merkoci, A., Nanoparticle-based lateral flow biosensors. Biosensors \& Bioelectronics 2015, 73, 47-63.

53. Liao, J. Y.; Li, H., Lateral flow immunodipstick for visual detection of aflatoxin B-1 in food using immuno-nanoparticles composed of a silver core and a gold shell. Microchimica Acta 2010, $171(3-4), 289-295$.

54. Tang, D.; Sauceda, J. C.; Lin, Z.; Ott, S.; Basova, E.; Goryacheva, I.; Biselli, S.; Lin, J.; Niessner, R.; Knopp, D., Magnetic nanogold microspheres-based lateral-flow immunodipstick for rapid detection of aflatoxin B-2 in food. Biosensors \& Bioelectronics 2009, 25 (2), 514-518.

55. (a) Berlina, A. N.; Taranova, N. A.; Zherdev, A. V.; Vengerov, Y. Y.; Dzantiev, B. B., Quantum dot-based lateral flow immunoassay for detection of chloramphenicol in milk. Analytical and Bioanalytical Chemistry 2013, 405 (14), 4997-5000; (b) Chen, R.; Li, H.; Zhang, H.; Zhang, S. X.; Shi, W. M.; Shen, J. Z.; Wang, Z. H., Development of a lateral flow fluorescent microsphere immunoassay for the determination of sulfamethazine in milk. Analytical and Bioanalytical Chemistry 2013, 405 (21), 6783-6789; (c) Dudek, M. M.; Kent, N. J.; Gu, P.; Fan, Z. H.; Killard, A. J., Development of a fluorescent method for detecting the onset of coagulation in human plasma on microstructured lateral flow platforms. Analyst 2011, 136 (9), 1816-1825; (d) Juntunen, E.; Myyrylainen, T.; Salminen, T.; Soukka, T.; Pettersson, K., Performance of fluorescent europium(III) nanoparticles and colloidal gold reporters in lateral flow bioaffinity assay. Analytical Biochemistry 2012, 428 (1), 31-38; (e) Leonardi, G. P.; Wilson, A. M.; Zuretti, A. R., Comparison of conventional lateral-flow assays and a new fluorescent immunoassay to detect influenza viruses. Journal of Virological Methods 2013, 189 (2), 379-382; (f) Li, X. P.; Lu, D. L.; Sheng, Z. H.; Chen, K.; Guo, X. B.; Jin, M. L.; Han, H. Y., A fast and sensitive immunoassay of avian influenza virus based on label-free quantum dot probe and lateral flow test strip. Talanta 2012, 100, 1-6; (g) Nabatiyan, A.; Baumann, M. A.; Parpia, Z.; Kelso, D., A Lateral Flow-Based Ultra-Sensitive p24 HIV Assay Utilizing Fluorescent Microparticles. JaidsJournal of Acquired Immune Deficiency Syndromes 2010, 53 (1), 55-61; (h) Wang, Y. H.; Nugen, S. R., Development of fluorescent nanoparticle-labeled lateral flow assay for the detection of nucleic acids. Biomedical Microdevices 2013, 15 (5), 751-758.

56. (a) Campion, A.; Kambhampati, P., Surface-enhanced Raman scattering. Chemical Society Reviews 1998, 27 (4), 241-250; (b) Kneipp, K.; Wang, Y.; Kneipp, H.; Perelman, L. T.; Itzkan, I.; Dasari, R.; Feld, M. S., Single molecule detection using surface-enhanced Raman 
scattering (SERS). Physical Review Letters 1997, 78 (9), 1667-1670; (c) Nie, S. M.; Emery, S. R., Probing single molecules and single nanoparticles by surface-enhanced Raman scattering. Science 1997, 275 (5303), 1102-1106.

57. (a) Kneipp, K.; Kneipp, H.; Itzkan, I.; Dasari, R. R.; Feld, M. S., Ultrasensitive chemical analysis by Raman spectroscopy. Chemical Reviews 1999, 99 (10), 2957-+; (b) Sharma, B.; Frontiera, R. R.; Henry, A. I.; Ringe, E.; Van Duyne, R. P., SERS: Materials, applications, and the future. Materials Today 2012, 15 (1-2), 16-25; (c) Stiles, P. L.; Dieringer, J. A.; Shah, N. C.; Van Duyne, R. R., Surface-Enhanced Raman Spectroscopy. Annual Review of Analytical Chemistry 2008, 1, 601-626.

58. Gao, X. F.; Zheng, P.; Kasani, S.; Wu, S.; Yang, F.; Lewis, S.; Nayeem, S.; EnglerChiurazzi, E. B.; Wigginton, J. G.; Simpkins, J. W.; Wu, N. Q., Paper-Based Surface-Enhanced Raman Scattering Lateral Flow Strip for Detection of Neuron-Specific Enolase in Blood Plasma. Analytical Chemistry 2017, 89 (18), 10104-10110.

59. (a) Kawaguchi, T.; Komatsu, S.; Ichikawa, D.; Tsujiura, M.; Takeshita, H.; Hirajima, S.; Miyamae, M.; Okajima, W.; Ohashi, T.; Imamura, T.; Kiuchi, J.; Konishi, H.; Shiozaki, A.; Okamoto, K.; Otsuji, E., Circulating MicroRNAs: A Next-Generation Clinical Biomarker for Digestive System Cancers. International Journal of Molecular Sciences 2016, 17 (9); (b) Swarup, V.; Rajeswari, M. R., Circulating (cell-free) nucleic acids - A promising, non-invasive tool for early detection of several human diseases. Febs Letters 2007, 581 (5), 795-799.

60. (a) Chen, X.; Ba, Y.; Ma, L. J.; Cai, X.; Yin, Y.; Wang, K. H.; Guo, J. G.; Zhang, Y. J.; Chen, J. N.; Guo, X.; Li, Q. B.; Li, X. Y.; Wang, W. J.; Zhang, Y.; Wang, J.; Jiang, X. Y.; Xiang, Y.; Xu, C.; Zheng, P. P.; Zhang, J. B.; Li, R. Q.; Zhang, H. J.; Shang, X. B.; Gong, T.; Ning, G.; Wang, J.; Zen, K.; Zhang, J. F.; Zhang, C. Y., Characterization of microRNAs in serum: a novel class of biomarkers for diagnosis of cancer and other diseases. Cell Research 2008, 18 (10), 997 1006; (b) Kosaka, N.; Iguchi, H.; Ochiya, T., Circulating microRNA in body fluid: a new potential biomarker for cancer diagnosis and prognosis. Cancer Science 2010, 101 (10), 20872092.

61. (a) Anker, P.; Stroun, M., Progress in the knowledge of circulating nucleic acids: Plasma RNA is particle-associated. Can it become a general detection marker for a cancer blood test? Clinical Chemistry 2002, 48 (8), 1210-1211; (b) Baraniskin, A.; Nopel-Dunnebacke, S.; Ahrens, M.; Jensen, S. G.; Zollner, H.; Maghnouj, A.; Wos, A.; Mayerle, J.; Munding, J.; Kost, D.; 
Reinacher-Schick, A.; Liffers, S.; Schroers, R.; Chromik, A. M.; Meyer, H. E.; Uhl, W.; KleinScory, S.; Weiss, F. U.; Stephan, C.; Schwarte-Waldhoff, I.; Lerch, M. M.; Tannapfel, A.; Schmiegel, W.; Andersen, C. L.; Hahn, S. A., Circulating U2 small nuclear RNA fragments as a novel diagnostic biomarker for pancreatic and colorectal adenocarcinoma. International Journal of Cancer 2013, 132 (2), E48-E57; (c) Bremnes, R. M.; Sirera, R.; Camps, C., Circulating tumour-derived DNA and RNA markers in blood: a tool for early detection, diagnostics, and follow-up? Lung Cancer 2005, 49 (1), 1-12.

62. (a) Heegaard, N. H. H.; Schetter, A. J.; Welsh, J. A.; Yoneda, M.; Bowman, E. D.; Harris, C. C., Circulating micro-RNA expression profiles in early stage nonsmall cell lung cancer. International Journal of Cancer 2012, 130 (6), 1378-1386; (b) Zhu, W.; Qin, W.; Atasoy, U.; Sauter, E. R., Circulating microRNAs in breast cancer and healthy subjects. BMC research notes 2009, 2 (1), 89.

63. Weiland, M.; Gao, X.-H.; Zhou, L.; Mi, Q.-S., Small RNAs have a large impact: circulating microRNAs as biomarkers for human diseases. $R N A$ biology 2012, 9 (6), 850-859.

64. Kenworthy, A. K., Imaging protein-protein interactions using fluorescence resonance energy transfer microscopy. Methods 2001, 24 (3), 289-296.

65. Chen, X.; Conti, P. S.; Moats, R. A., In vivo near-infrared fluorescence imaging of integrin $\alpha v \beta 3$ in brain tumor xenografts. Cancer research 2004, 64 (21), 8009-8014.

66. (a) Lian, W.; Litherland, S. A.; Badrane, H.; Tan, W.; Wu, D.; Baker, H. V.; Gulig, P. A.; Lim, D. V.; Jin, S., Ultrasensitive detection of biomolecules with fluorescent dye-doped nanoparticles. Analytical biochemistry 2004, 334 (1), 135-144; (b) Smith, J. E.; Wang, L.; Tan, W., Bioconjugated silica-coated nanoparticles for bioseparation and bioanalysis. Trac Trends in Analytical Chemistry 2006, 25 (9), 848-855.

67. (a) Deng, T.; Li, J. S.; Jiang, J. H.; Shen, G. L.; Yu, R. Q., Preparation of Near-IR Fluorescent Nanoparticles for Fluorescence-Anisotropy-Based Immunoagglutination Assay in Whole Blood. Advanced Functional Materials 2006, 16 (16), 2147-2155; (b) Sharma, P.; Bengtsson, N. E.; Walter, G. A.; Sohn, H. B.; Zhou, G.; Iwakuma, N.; Zeng, H.; Grobmyer, S. R.; Scott, E. W.; Moudgil, B. M., Gadolinium-doped silica nanoparticles encapsulating indocyanine green for near infrared and magnetic resonance imaging. Small 2012, 8 (18), 2856-2868; (c) Sriramulu, D.; Reed, E. L.; Annamalai, M.; Venkatesan, T. V.; Valiyaveettil, S., Synthesis and characterization of superhydrophobic, self-cleaning NIR-reflective silica nanoparticles. Scientific 
reports 2016, 6, 35993.

68. Xu, H.; Mao, X.; Zeng, Q.; Wang, S.; Kawde, A.-N.; Liu, G., Aptamer-functionalized gold nanoparticles as probes in a dry-reagent strip biosensor for protein analysis. Analytical Chemistry 2008, 81 (2), 669-675.

69. Xu, H.; Chen, J.; Birrenkott, J.; Zhao, J. X.; Takalkar, S.; Baryeh, K.; Liu, G., Goldnanoparticle-decorated silica nanorods for sensitive visual detection of proteins. Analytical chemistry 2014, 86 (15), 7351-7359.

70. (a) Allegra, A.; Alonci, A.; Campo, S.; Penna, G.; Petrungaro, A.; Gerace, D.; Musolino, C., Circulating microRNAs: new biomarkers in diagnosis, prognosis and treatment of cancer. International journal of oncology 2012, 41 (6), 1897-1912; (b) Corcoran, C.; Rani, S.; O'Driscoll, L., miR-34a is an intracellular and exosomal predictive biomarker for response to docetaxel with clinical relevance to prostate cancer progression. The Prostate 2014, 74 (13), 1320-1334; (c) Jansen, C.; Eischeid, H.; Goertzen, J.; Schierwagen, R.; Anadol, E.; Strassburg, C. P.; Sauerbruch, T.; Odenthal, M.; Trebicka, J., The role of miRNA-34a as a prognostic biomarker for cirrhotic patients with portal hypertension receiving TIPS. PloS one 2014, 9 (7), e103779; (d) Li, X.; Khanna, A.; Li, N.; Wang, E., Circulatory miR-34a as an RNA-based, noninvasive biomarker for brain aging. Aging (Albany NY) 2011, 3 (10), 985.

71. Várallyay, É.; Burgyán, J.; Havelda, Z., MicroRNA detection by northern blotting using locked nucleic acid probes. Nature protocols 2008, 3 (2), 190.

72. (a) Chen, C.; Ridzon, D. A.; Broomer, A. J.; Zhou, Z.; Lee, D. H.; Nguyen, J. T.; Barbisin, M.; Xu, N. L.; Mahuvakar, V. R.; Andersen, M. R., Real-time quantification of microRNAs by stem-loop RT-PCR. Nucleic acids research 2005, 33 (20), e179-e179; (b) Kroh, E. M.; Parkin, R. K.; Mitchell, P. S.; Tewari, M., Analysis of circulating microRNA biomarkers in plasma and serum using quantitative reverse transcription-PCR (qRT-PCR). Methods 2010, 50 (4), 298-301; (c) Zhi, F.; Chen, X.; Wang, S.; Xia, X.; Shi, Y.; Guan, W.; Shao, N.; Qu, H.; Yang, C.; Zhang, Y., The use of hsa-miR-21, hsa-miR-181b and hsa-miR-106a as prognostic indicators of astrocytoma. European Journal of Cancer 2010, 46 (9), 1640-1649.

73. (a) Konishi, H.; Ichikawa, D.; Komatsu, S.; Shiozaki, A.; Tsujiura, M.; Takeshita, H.; Morimura, R.; Nagata, H.; Arita, T.; Kawaguchi, T., Detection of gastric cancer-associated microRNAs on microRNA microarray comparing pre-and post-operative plasma. British journal of cancer 2012, 106 (4), 740; (b) Wu, F.; Zhang, S.; Dassopoulos, T.; Harris, M. L.; Bayless, T. 
M.; Meltzer, S. J.; Brant, S. R.; Kwon, J. H., Identification of microRNAs associated with ileal and colonic Crohn's disease. Inflammatory bowel diseases 2010, 16 (10), 1729-1738; (c) Yang, N.; Kaur, S.; Volinia, S.; Greshock, J.; Lassus, H.; Hasegawa, K.; Liang, S.; Leminen, A.; Deng, S.; Smith, L., MicroRNA microarray identifies Let-7i as a novel biomarker and therapeutic target in human epithelial ovarian cancer. Cancer research 2008, 68 (24), 10307-10314.

74. Shen, W.; Deng, H.; Ren, Y.; Gao, Z., A real-time colorimetric assay for label-free detection of microRNAs down to sub-femtomolar levels. Chemical Communications 2013, 49 (43), 4959-4961.

75. (a) Almlie, C. K.; Larkey, N. E.; Burrows, S. M., Fluorescent microRNA biosensors: a comparison of signal generation to quenching. Analytical Methods 2015, 7 (17), 7296-7310; (b) Ryoo, S.-R.; Lee, J.; Yeo, J.; Na, H.-K.; Kim, Y.-K.; Jang, H.; Lee, J. H.; Han, S. W.; Lee, Y.; Kim, V. N., Quantitative and multiplexed microRNA sensing in living cells based on peptide nucleic acid and nano graphene oxide (PANGO). ACS nano 2013, 7 (7), 5882-5891; (c) Zhang, Y.; Zhang, C.-y., Sensitive detection of microRNA with isothermal amplification and a singlequantum-dot-based nanosensor. Analytical chemistry 2011, 84 (1), 224-231.

76. (a) Ding, X.; Yan, Y.; Li, S.; Zhang, Y.; Cheng, W.; Cheng, Q.; Ding, S., Surface plasmon resonance biosensor for highly sensitive detection of microRNA based on DNA super-sandwich assemblies and streptavidin signal amplification. Analytica chimica acta 2015, 874, 59-65; (b) Li, J.; Lei, P.; Ding, S.; Zhang, Y.; Yang, J.; Cheng, Q.; Yan, Y., An enzyme-free surface plasmon resonance biosensor for real-time detecting microRNA based on allosteric effect of mismatched catalytic hairpin assembly. Biosensors and Bioelectronics 2016, 77, 435-441.

77. (a) Johnson, B. N.; Mutharasan, R., Sample preparation-free, real-time detection of microRNA in human serum using piezoelectric cantilever biosensors at attomole level. Analytical chemistry 2012, 84 (23), 10426-10436; (b) Sun, E.; Wang, L.; Zhou, X.; Ma, C.; Sun, Y.; Lei, M.; Lu, B.; Han, R., Graphene oxide/DNA-decorated electrode for the fabrication of microRNA biosensor. RSC Advances 2015, 5 (85), 69334-69338; (c) Wen, Y.; Liu, G.; Pei, H.; Li, L.; Xu, Q.; Liang, W.; Li, Y.; Xu, L.; Ren, S.; Fan, C., DNA nanostructure-based ultrasensitive electrochemical microRNA biosensor. Methods 2013, 64 (3), 276-282.

78. (a) Congur, G.; Eksin, E.; Erdem, A., Impedimetric detection of microRNA at graphene oxide modified sensors. Electrochimica Acta 2015, 172, 20-27; (b) Ren, Y.; Deng, H.; Shen, W.; Gao, Z., A highly sensitive and selective electrochemical biosensor for direct detection of 
microRNAs in serum. Analytical chemistry 2013, 85 (9), 4784-4789.

79. (a) Zheng, P.; Li, M.; Jurevic, R.; Cushing, S. K.; Liu, Y.; Wu, N., A gold nanohole array based surface-enhanced Raman scattering biosensor for detection of silver (I) and mercury (II) in human saliva. Nanoscale 2015, 7 (25), 11005-11012; (b) Zheng, P.; Shi, X.; Curtin, K.; Yang, F.; $\mathrm{Wu}, \mathrm{N}$., Detection of mercury (II) with a surface-enhanced Raman scattering sensor based on functionalized gold nanoparticles. Materials Research Express 2017, 4 (5), 055017.

80. Farh, K. K.-H.; Grimson, A.; Jan, C.; Lewis, B. P.; Johnston, W. K.; Lim, L. P.; Burge, C. B.; Bartel, D. P., The widespread impact of mammalian MicroRNAs on mRNA repression and evolution. Science 2005, 310 (5755), 1817-1821.

81. Cissell, K. A.; Shrestha, S.; Deo, S. K., MicroRNA detection: challenges for the analytical chemist. ACS Publications: 2007.

82. (a) Esquela-Kerscher, A.; Slack, F. J., Oncomirs-microRNAs with a role in cancer. Nature reviews cancer 2006, 6 (4), 259; (b) Tavazoie, S. F.; Alarcón, C.; Oskarsson, T.; Padua, D.; Wang, Q.; Bos, P. D.; Gerald, W. L.; Massagué, J., Endogenous human microRNAs that suppress breast cancer metastasis. Nature 2008, 451 (7175), 147.

83. Bustin, S., INVITED REVIEW Quantification of mRNA using real-time reverse transcription PCR (RT-PCR): trends and problems. Journal of molecular endocrinology 2002, 29, 23-39.

84. (a) Yang, S. W.; Vosch, T., Rapid detection of microRNA by a silver nanocluster DNA probe. Analytical chemistry 2011, 83 (18), 6935-6939; (b) Zhang, J.; Li, C.; Zhi, X.; Ramón, G. A.; Liu, Y.; Zhang, C.; Pan, F.; Cui, D., Hairpin DNA-templated silver nanoclusters as novel beacons in strand displacement amplification for microRNA detection. Analytical chemistry 2015, 88 (2), 1294-1302; (c) Zhou, W.; Li, Q.; Liu, H.; Yang, J.; Liu, D., Building electromagnetic hot spots in living cells via target-triggered nanoparticle dimerization. ACS nano 2017, 11 (4), 3532-3541.

85. (a) Jonstrup, S. P.; Koch, J.; Kjems, J., A microRNA detection system based on padlock probes and rolling circle amplification. Rna 2006, 12 (9), 1747-1752; (b) Zhang, J.; Li, Z.; Wang, H.; Wang, Y.; Jia, H.; Yan, J., Ultrasensitive quantification of mature microRNAs by real-time PCR based on ligation of a ribonucleotide-modified DNA probe. Chemical Communications 2011, 47 (33), 9465-9467; (c) Zhu, W.; Su, X.; Gao, X.; Dai, Z.; Zou, X., A label-free and PCRfree electrochemical assay for multiplexed microRNA profiles by ligase chain reaction coupling 
with quantum dots barcodes. Biosensors and Bioelectronics 2014, 53, 414-419.

86. (a) Dean, F. B.; Nelson, J. R.; Giesler, T. L.; Lasken, R. S., Rapid amplification of plasmid and phage DNA using phi29 DNA polymerase and multiply-primed rolling circle amplification. Genome research 2001, 11 (6), 1095-1099; (b) Lizardi, P. M.; Huang, X.; Zhu, Z.; Bray-Ward, P.; Thomas, D. C.; Ward, D. C., Mutation detection and single-molecule counting using isothermal rolling-circle amplification. Nature genetics 1998, 19 (3), 225.

87. (a) Jia, H.; Li, Z.; Liu, C.; Cheng, Y., Ultrasensitive detection of microRNAs by exponential isothermal amplification. Angewandte Chemie International Edition 2010, 49 (32), 5498-5501; (b) Liu, H.; Tian, T.; Zhang, Y.; Ding, L.; Yu, J.; Yan, M., Sensitive and rapid detection of microRNAs using hairpin probes-mediated exponential isothermal amplification. Biosensors and Bioelectronics 2017, 89, 710-714.

88. (a) Lin, X.; Zhang, C.; Huang, Y.; Zhu, Z.; Chen, X.; Yang, C. J., Backbone-modified molecular beacons for highly sensitive and selective detection of microRNAs based on duplex specific nuclease signal amplification. Chemical Communications 2013, 49 (65), 7243-7245; (b) Yin, B.-C.; Liu, Y.-Q.; Ye, B.-C., One-step, multiplexed fluorescence detection of microRNAs based on duplex-specific nuclease signal amplification. Journal of the American Chemical Society 2012, 134 (11), 5064-5067.

89. (a) Chen, Y.-X.; Huang, K.-J.; Niu, K.-X., Recent advances in signal amplification strategy based on oligonucleotide and nanomaterials for microRNA detection-a review. Biosensors and Bioelectronics 2018, 99, 612-624; (b) Tian, T.; Wang, J.; Zhou, X., A review: microRNA detection methods. Organic \& biomolecular chemistry 2015, 13 (8), 2226-2238.

90. Ali, M. M.; Li, F.; Zhang, Z.; Zhang, K.; Kang, D.-K.; Ankrum, J. A.; Le, X. C.; Zhao, W., Rolling circle amplification: a versatile tool for chemical biology, materials science and medicine. Chemical Society Reviews 2014, 43 (10), 3324-3341.

91. Jiang, J.; Zhang, B.; Zhang, C.; Guan, Y., A Novel Design Combining Isothermal Exponential Amplification and Gold-Nanoparticles Visualization for Rapid Detection of miRNAs. International journal of molecular sciences 2018, 19 (11), 3374.

92. Qiu, X.; Zhang, H.; Yu, H.; Jiang, T.; Luo, Y., Duplex-specific nuclease-mediated bioanalysis. Trends in biotechnology 2015, 33 (3), 180-188.

93. (a) Anisimova, V. E.; Rebrikov, D. V.; Shagin, D. A.; Kozhemyako, V. B.; Menzorova, N. I.; Staroverov, D. B.; Ziganshin, R.; Vagner, L. L.; Rasskazov, V. A.; Lukyanov, S. A., Isolation, 
characterization and molecular cloning of duplex-specific nuclease from the hepatopancreas of the Kamchatka crab. BMC biochemistry 2008, 9 (1), 14; (b) Fu, C.; Liu, C.; Wang, S.; Luo, F.; Lin, Z.; Chen, G., A signal-on homogeneous electrochemical biosensor for sequence-specific microRNA based on duplex-specific nuclease-assisted target recycling amplification. Analytical Methods 2016, 8 (39), 7034-7039; (c) Zhang, H.; Wang, K.; Bu, S.; Li, Z.; Ju, C.; Wan, J., Colorimetric detection of microRNA based on DNAzyme and nuclease-assisted catalytic hairpin assembly signal amplification. Molecular and cellular probes 2018, 38, 13-18.

94. Wang, Q.; Li, R.-D.; Yin, B.-C.; Ye, B.-C., Colorimetric detection of sequence-specific microRNA based on duplex-specific nuclease-assisted nanoparticle amplification. Analyst 2015, 140 (18), 6306-6312.

95. Xi, Q.; Zhou, D.-M.; Kan, Y.-Y.; Ge, J.; Wu, Z.-K.; Yu, R.-Q.; Jiang, J.-H., Highly sensitive and selective strategy for microRNA detection based on WS2 nanosheet mediated fluorescence quenching and duplex-specific nuclease signal amplification. Analytical chemistry 2014, 86 (3), 1361-1365.

96. (a) Liu, L.; Gao, Y.; Liu, H.; Xia, N., An ultrasensitive electrochemical miRNAs sensor based on miRNAs-initiated cleavage of DNA by duplex-specific nuclease and signal amplification of enzyme plus redox cycling reaction. Sensors and Actuators B: Chemical 2015, 208, 137-142; (b) Zhang, J.; Wu, D.-Z.; Cai, S.-X.; Chen, M.; Xia, Y.-K.; Wu, F.; Chen, J.-H., An immobilization-free electrochemical impedance biosensor based on duplex-specific nuclease assisted target recycling for amplified detection of microRNA. Biosensors and Bioelectronics 2016, 75, 452-457. 


\section{Appendix:}

\section{Completed manuscripts:}

1. Enabling Direct Protein Detection in a Drop of Whole Blood with an "On-Strip" Plasma Separation Unit in a Paper-Based Lateral Flow Strip.

Authors: Xuefei Gao, Sujan Kasani and Nianqiang Wu

2. Incorporation of Nanostructured Plasmonic Chip into Paper-based Lateral Flow Strip for S$100 \beta$ Detection in Whole Blood.

Authors: Xuefei Gao, Sujan Kasani, Peng Zheng, Kathrine Curtin, Jennifer Boryczka, and Nianqiang $\mathrm{Wu}$

3. Comparative Visible-light and Near-Infrared Fluorescence Detection of microRNA in Blood Plasma with Paper-based Lateral Flow Test Strips

Authors: Xuefei Gao, Peng Zheng, Feng Yang, Elizabeth Engler-Chiurazzi, James W. Simpkins and Nianqiang $\mathrm{Wu}$

4. Nuclease-assisted Amplification Technique Modified Paper-based Test Strip for Ultrasensitive Detection of MicroRNA-34 in Blood Plasma.

Authors: Xuefei Gao, Sujan Kasani, Peng Zheng, Kathrine Curtin, Jennifer Boryczka and Nianqiang $\mathrm{Wu}$

\section{Publications:}

1. $\underline{\text { Xuefei Gao }}$, Peng Zheng, Sujan Kasani, Steven Wu, Feng Yang, Sara Lewis, Sara Nayeem, Elizabeth B. Engler-Chiurazzi, Jane G. Wigginton, James W. Simpkins and Nianqiang Wu*. Paper-Based Surface-Enhanced Raman Scattering Lateral Flow Strip for Detection of NeuronSpecific Enolase in Blood Plasma, Analytical Chemistry, 2017, 89, 10104-10110.

2. Xuefei Gao and Nianqiang Wu*, Smartphone-Based Sensors, The Electrochemical Society Interface, 2016, 25, 79-81.

3. Xuefei Gao, Liping $\mathrm{Xu}^{*}$, Tingting $\mathrm{Wu}$, Yongqiang $\mathrm{Wu}$, Xinlei Ma and Xueji Zhang*. An Enzyme-Amplified Lateral Flow Strip Biosensor for Visual Detection of MicroRNA-224, Talanta, 2016, 146, 648-654.

4. $\underline{\text { Xuefei Gao }}$, Li-Ping Xu*, Zhongxin Xue, Lin Feng, Jitao Peng, Yongqiang Wen, Shutao Wang* and Xueji Zhang*, Dual-Scaled Porous Nitrocellulose Membranes with Underwater Superoleophobicity for Highly Efficient Oil/Water Separation, Advanced Materials, 2014, 26, 
1771-1775.

5. $\underline{\text { Xuefei Gao }}$, Hui Xu, Meenu Baloda, Anant S. Gurung, Li-Ping Xu, Tao Wang, Xueji Zhang* and Guodong Liu*. Visual Detection of MicroRNA with Lateral Flow Nucleic Acid Biosensor, Biosensors \& Bioelectronics, 2014, 54, 578-584.

6. $\underline{\text { Xuefei Gao }}$, Li-Ping Xu*, Shu-Feng Zhou, Guodong Liu and Xueji Zhang*, Recent Advances in Nanoparticles-Based Lateral Flow Biosensors, American Journal Biomedical Sciences, 2014, $6,41-57$. 